\title{
PROPERTIES OF THE YOUNGEST PROTOSTARS IN PERSEUS, SERPENS, AND OPHIUCHUS
}

\author{
Melissa L. Enoch ${ }^{1}$, Neal J. Evans II $^{2}$, Anneila I. Sargent ${ }^{3}$, and Jason Glenn ${ }^{4}$ \\ ${ }^{1}$ Department of Astronomy, University of California, Berkeley, CA 94720, USA; menoch@astro.berkeley.edu \\ 2 The University of Texas at Austin, Astronomy Department, 1 University Station C1400, Austin, TX 78712-0259, USA \\ ${ }^{3}$ Division of Physics, Mathematics \& Astronomy, California Institute of Technology, Pasadena, CA 91125, USA \\ ${ }^{4}$ Center for Astrophysics and Space Astronomy, 389-UCB, University of Colorado, Boulder, CO 80309, USA \\ Received 2008 July 24; accepted 2008 September 23; published 2009 February 23
}

\begin{abstract}
We present an unbiased census of deeply embedded protostars in Perseus, Serpens, and Ophiuchus, assembled by combining large-scale $1.1 \mathrm{~mm}$ Bolocam continuum and Spitzer Legacy surveys. We identify protostellar candidates based on their mid-infrared (mid-IR) properties, correlate their positions with $1.1 \mathrm{~mm}$ core positions from Enoch et al. in 2006 and 2007, and Young et al. in 2006, and construct well-sampled spectral energy distributions using our extensive wavelength coverage $(\lambda=1.25-1100 \mu \mathrm{m})$. Source classification based on the bolometric temperature yields a total of 39 Class 0 and 89 Class I sources in the three-cloud sample. We compare to protostellar evolutionary models using the bolometric temperature-luminosity diagram, finding a population of low-luminosity Class I sources that are inconsistent with constant or monotonically decreasing mass accretion rates. This result argues strongly for episodic accretion during the Class I phase, with more than $50 \%$ of sources in a "sub-Shu" $\left(d M / d t<10^{-6} M_{\odot} \mathrm{yr}^{-1}\right)$ accretion state. Average spectra are compared to protostellar radiative transfer models, which match the observed spectra fairly well in Stage 0, but predict too much near-IR and too little mid-IR flux in Stage I. Finally, the relative number of Class 0 and Class I sources is used to estimate the lifetime of the Class 0 phase; the three-cloud average yields a Class 0 lifetime of $1.7 \pm 0.3 \times 10^{5} \mathrm{yr}$, ruling out an extremely rapid early accretion phase. Correcting photometry for extinction results in a somewhat shorter lifetime $\left(1.1 \times 10^{5} \mathrm{yr}\right)$. In Ophiuchus, however, we find very few Class 0 sources $\left(N_{\text {Class } 0} / N_{\text {Class I }} \sim 0.1-0.2\right)$, similar to previous studies of that cloud. The observations suggest a consistent picture of nearly constant average accretion rate through the entire embedded phase, with accretion becoming episodic by at least the Class I stage, and possibly earlier.
\end{abstract}

Key words: infrared: ISM - ISM: clouds - ISM: individual (Perseus, Serpens, Ophiuchus) - stars: formation submillimeter

Online-only material: color figures, extended figure

\section{INTRODUCTION}

The problem of how low-mass stars like the Sun form has been studied extensively over the last few decades. Compared to more evolved protostars and pre-main-sequence objects, however, the earliest stages of the star formation process, from the formation of dense cores through the main mass accretion phase, are relatively poorly understood. This lack of information is due in large part to the difficulty of observing young, deeply embedded sources, which are shrouded within dense protostellar envelopes and only observable via reprocessed emission at mid-infrared (mid-IR) to millimeter wavelengths. Furthermore, most previous observations of deeply embedded objects have naturally focused on a small number of very bright or wellknown sources, due to the sensitivity and resolution limitations of long-wavelength surveys. Understanding the formation of typical stars requires complete samples of young objects, over molecular cloud scales.

Currently, the details of the early evolution of protostellar sources are extremely uncertain, including mass accretion rates during the Class 0 and Class I phases. In addition, measurements of the timescales associated with the earliest stages vary considerably, ranging from $10^{5}$ to $10^{7} \mathrm{yr}$ for prestellar cores (Ward-Thompson et al. 2007) and from $10^{4}$ to a few $\times 10^{5} \mathrm{yr}$ for Class 0 (André \& Montmerle 1994; Visser et al. 2002). In fact, the association of Class 0 and Class I with distinct evolutionary stages is still a matter of debate (e.g., Jayawardhana et al. 2001). Large surveys at mid-IR to millimeter wavelengths, where the spectral energy distributions (SEDs) of embedded sources peak, are essential for understanding how protostars evolve through their earliest stages. In addition to providing complete samples of young sources, large surveys are also important for characterizing variations in the star formation process with environment.

With a few notable recent exceptions (Jørgensen et al. 2007; Hatchell et al. 2007b), previous samples of very young protostars have typically been compiled from many different surveys, and suffered from systematics, unquantified environmental effects, and small number statistics. We recently completed largescale $1.1 \mathrm{~mm}$ continuum surveys of Perseus, Serpens, and Ophiuchus with Bolocam at the Caltech Submillimeter Observatory (CSO). Maps have a resolution of $31^{\prime \prime}$ and cover $7.5 \mathrm{deg}^{2}$ in Perseus (140 $\mathrm{pc}^{2}$ at our adopted cloud distance of $d=250 \mathrm{pc}$ ), $10.8 \mathrm{deg}^{2}$ in Ophiuchus (50 $\mathrm{pc}^{2}$ at $d=125 \mathrm{pc}$ ), and $1.5 \mathrm{deg}^{2}$ in Serpens (30 $\mathrm{pc}^{2}$ at $d=260 \mathrm{pc}$ ) (Enoch et al. 2006; Young et al. 2006; Enoch et al. 2007, hereafter Papers I, II, and III, respectively). These Bolocam surveys complement large Spitzer Space Telescope IRAC and MIPS maps of the same clouds from the "From Molecular Cores to Planet-forming Disks" Spitzer Legacy program ("Cores to Disks" or c2d; Evans et al. 2003).

Millimeter emission traces the dust in dense starless cores and protostellar envelopes, and provides a measure of core and envelope properties, including sizes, masses, and spatial distribution. Spitzer IRAC and MIPS observations are complementary in that they provide information about the properties of 
any young protostars embedded within dense cores. Combining these data enables us to assemble a mass limited, unbiased census of the youngest star-forming objects in three different environments, including prestellar cores, Class 0 , and Class I protostars.

In Paper III, we looked at how the global cloud environment influences the properties of star-forming cores (Enoch et al. 2007). In a companion paper to this work (Enoch et al. 2008), we examine the properties of prestellar and protostellar cores in Perseus, Serpens, and Ophiuchus, focusing on the prestellar core mass distribution and the lifetime of prestellar cores. A similar analysis comparing the c2d Spitzer data to large SCUBA maps of Perseus and Ophiuchus has recently been carried out by Jørgensen et al. (2007, 2008), focusing on the difference between starless and protostellar cores, as well as cloud properties such as the star formation efficiency and how it varies with spatial clustering. We follow Di Francesco et al. (2007) in defining millimeter cores containing a compact luminous internal source (i.e., an embedded protostar) as "protostellar cores," regardless of whether the final object will be stellar or substellar in nature. We use "starless cores" to refer to dense cores without an internal luminosity source, and "prestellar cores" as starless cores that are likely to be gravitationally bound (see Enoch et al. 2008).

In this work, we exploit the combined power of millimeter and mid- to far-IR observations to study the evolution of young protostars embedded within the protostellar cores. Extensive wavelength coverage from $\lambda=1.25-1100 \mu \mathrm{m}$ allows us to trace the evolution of protostellar sources in their main mass accretion phase, from formation through the end of the embedded phase. We follow Robitaille et al. (2006) and Crapsi et al. (2008) in using "Stage" (e.g., Stages 0, I, II, III) to refer to a source's true physical nature, regardless of its observed properties, while the corresponding "Class" refers to the observational classification, typically based on the near- to mid-IR slope $\alpha_{\mathrm{IR}}$ (Adams et al. 1987; Greene et al. 1994) or on the bolometric temperature (Myers \& Ladd 1993).

We assume that Stage 0 and Stage I refer to an evolutionary sequence of embedded protostars with $M_{*}<M_{\text {env }}$ and $M_{*}>$ $M_{\text {env }}$, respectively (e.g., André 1994), where $M_{*}$ is the protostar mass and $M_{\text {env }}$ the envelope mass. Similarly, we assume that Stage II refers to pre-main-sequence stars with very little remaining envelope $\left(M_{\text {env }}<0.1 M_{\odot}\right.$; Crapsi et al. 2008). Our adopted definitions of various classes and stages are summarized in Table 1. Ideally, Class I would directly correspond to Stage I, etc., but as shown by Robitaille et al. (2006) and Crapsi et al. (2008), geometric effects can cause, for example, Stage II sources to be classified as Class I.

In Section 2 we describe the $1.1 \mathrm{~mm}$ and Spitzer IR data, including how protostellar candidates are identified and their association with $1.1 \mathrm{~mm}$ cores. We calculate bolometric luminosities and temperatures, envelope masses, and discuss completeness (Section 3). Protostellar classification methods are compared in Section 4. Spectral characteristics of Class 0, I, and II sources detected at $1.1 \mathrm{~mm}$ are discussed in Section 5, including selected individual sources (Section 5.2). In Section 6 we explore alternative classification schemes made possible by our large sample, and compare the average observed spectra to protostellar models in Section 7. Sources are placed on a bolometric temperatureluminosity diagram for comparison to protostellar evolutionary models and to study their luminosity evolution, mass accretion rates, and envelope evolution (Section 8). Finally, in Section 9 we calculate lifetimes for the Class 0 phase.

\section{COMBINING BOLOCAM AND SPITZER c2d DATA}

Both Bolocam $1.1 \mathrm{~mm}$ and Spitzer maps were designed to cover down to a visual extinction of $A_{V} \gtrsim 2$ mag in Perseus, $A_{V} \gtrsim 3 \mathrm{mag}$ in Ophiuchus, and $A_{V} \gtrsim 6 \mathrm{mag}$ in Serpens (Evans et al. 2003). The actual overlap in area between Bolocam and IRAC maps is shown in Figure 1 of Papers I, II, and III for Perseus, Ophiuchus, and Serpens, respectively. Catalogs listing c2d measured Spitzer fluxes of all sources in each of the three clouds, as well as near-IR fluxes for sources in the Two Micron All Sky Survey (2MASS) catalogs, are available through the Spitzer database (Evans et al. 2007). We utilize wavelength coverage from $\lambda=1.25$ to $1100 \mu \mathrm{m}$, using 2MASS (1.25, 1.65, $2.17 \mu \mathrm{m}), \operatorname{IRAC}(3.6,4.5,5.8,8.0 \mu \mathrm{m}), \operatorname{MIPS}(24,70$, $160 \mu \mathrm{m})$, and Bolocam $(1.1 \mathrm{~mm})$ data. Note that $160 \mu \mathrm{m}$ flux measurements are not included in the c 2 d delivery catalogs due to substantial uncertainties and incompleteness, but are included here when possible. Photometry at $160 \mu \mathrm{m}$ is discussed in Rebull et al. (2007) and Harvey et al. (2007b), where $160 \mu \mathrm{m}$ fluxes for point sources in Perseus and Serpens are also given.

Basic data papers describe the processing and analysis of the Spitzer IRAC and MIPS maps of Perseus, Serpens, and Ophiuchus, as well as presenting general properties of the sources in each cloud, such as color-color and color-magnitude diagrams (Jørgensen et al. 2006; Harvey et al. 2006, 2007b; Rebull et al. 2007). The young stellar object (YSO) population in Serpens is discussed in detail by Harvey et al. (2007a). Here, we are most interested in the young protostellar sources that are most likely to be embedded in the millimeter cores detected with Bolocam. For the following we will use the term "embedded protostar candidate" in general to encompass candidate Stage I and younger objects. Although it will become apparent in Section 2.1 that our criteria also pick up a number of Stage II sources, in general we focus on sources with evidence for a protostellar envelope (Stages 0 and I).

Figure 1 shows the result of combining Spitzer and Bolocam data, for a few examples of embedded protostellar sources in each cloud. Images are three-color Spitzer maps (8.0, 24, $70 \mu \mathrm{m}$ ), with $1.1 \mathrm{~mm}$ contours overlaid. The Bolocam ID of the associated $1.1 \mathrm{~mm}$ core is given in each panel, as well as the embedded source IDs from Tables 2-4. We use the 2MASS, IRAC, MIPS, and Bolocam data to construct complete SEDs from 2 to $1100 \mu \mathrm{m}$ for each candidate protostellar source, as shown in the lower panels (see also Section 3). Additional SHARC II $350 \mu \mathrm{m}$ fluxes (Wu et al. 2007) and SCUBA $850 \mu \mathrm{m}$ fluxes (Kirk et al. 2006, open circles) are included when available. Modified blackbody $\left(S_{v} \propto v^{\beta} B_{v}(T)\right)$ curves for a temperature of $15 \mathrm{~K}$ and $\beta=1$ are shown for reference in Figure 1 (dotted lines).

Protostellar sources in our sample may be isolated (e.g., Oph-Bolo 26) or lie in crowded regions (e.g., Per-Bolo 49). Approximately $20-50 \%$ of the time, more than one protostellar source lies within a single $1.1 \mathrm{~mm}$ core $(20 \%$ of the embedded protostar sample in Ophiuchus, $40 \%$ in Perseus, and 55\% in Serpens). Much of this difference is certainly due to the lower resolution of Bolocam ( $\left.31^{\prime \prime}\right)$ compared to Spitzer ( $7^{\prime \prime}$ at $24 \mu \mathrm{m}$ ), causing nearby Spitzer sources to be blended in the $1.1 \mathrm{~mm}$ map, although the envelopes detected at $1.1 \mathrm{~mm}$ are also physically more extended than the region emitting at Spitzer wavelengths. Sometimes the SEDs of such multiple sources look similar to each other (Per-emb 18 and 21 in the lower-left panel of Figure 1), and sometimes quite different (Per-emb 49 of the same panel). 

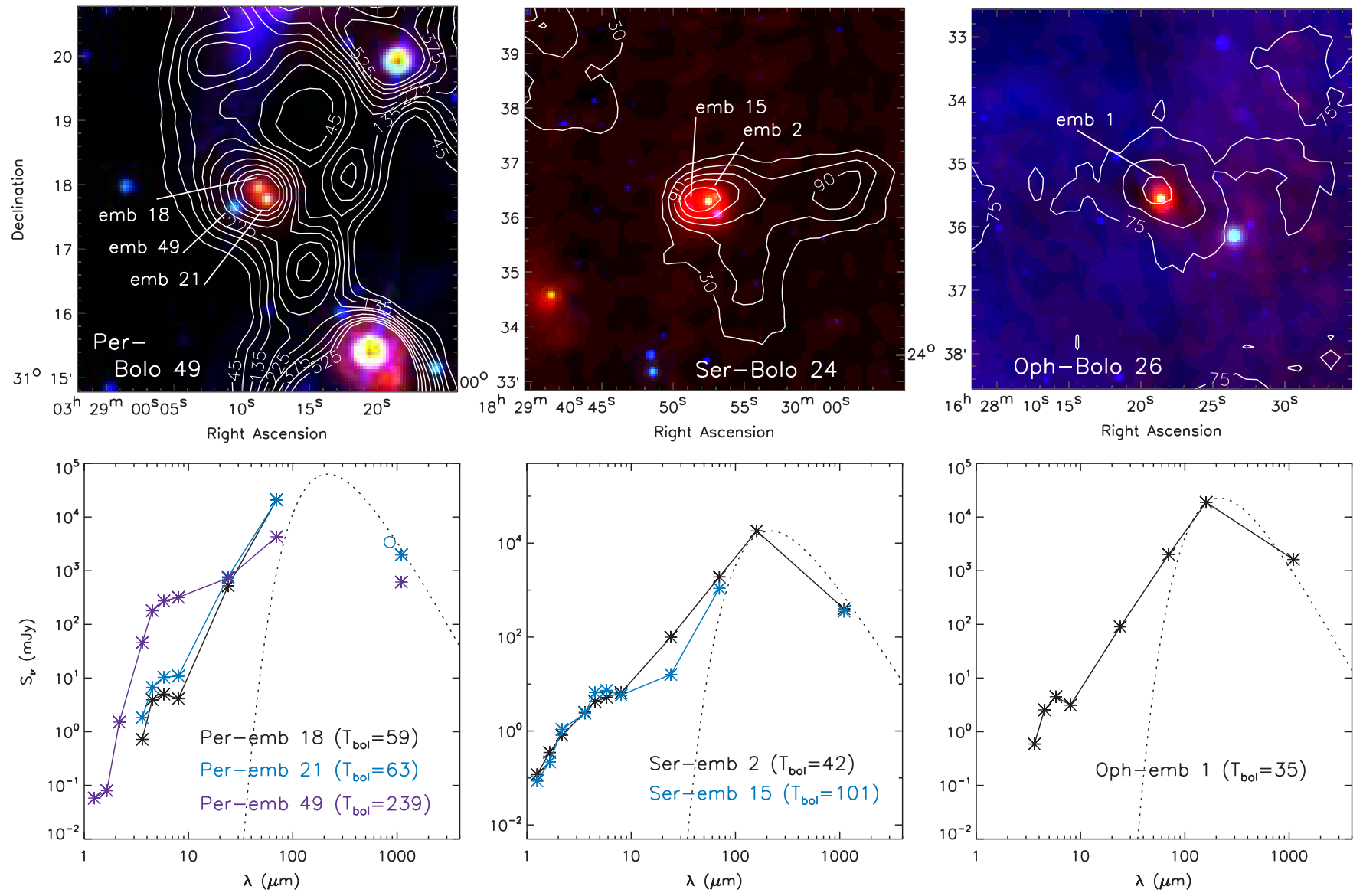

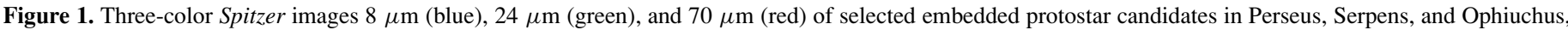

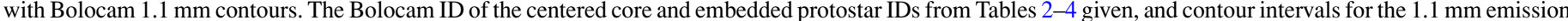

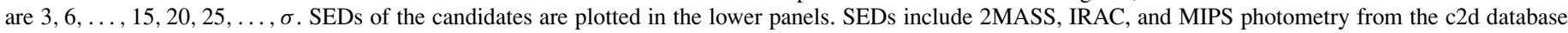

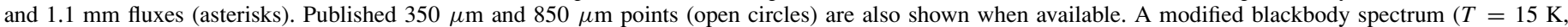
$\beta=1$ ) is overlaid for reference.

Although the coverage of the IRAC, MIPS, and Bolocam maps overlaps nearly perfectly for our purposes, there are a few cases in which embedded protostar candidates are outside the boundaries of the MIPS $70 \mu \mathrm{m}$ map (three sources in Serpens) or $1.1 \mathrm{~mm}$ map (one source in Perseus). In addition, a number of bright sources in each cloud are saturated in the $24 \mu \mathrm{m}$ or $70 \mu \mathrm{m}$ c $2 \mathrm{~d}$ maps. In these cases, we substitute IRAS $25 \mu \mathrm{m}$ or $60 \mu \mathrm{m}$ fluxes if the source does not appear to be blended in a visual inspection of the IRAS maps.

The $160 \mu \mathrm{m}$ maps are often saturated near bright sources and in regions of bright extended emission, such as near bright clusters of sources. Reliable $160 \mu \mathrm{m}$ fluxes are especially difficult to determine in crowded regions, due both to the large beam size $\left(40^{\prime \prime}\right)$ and to saturation issues. The lack of $160 \mu \mathrm{m}$ data is most problematic in Ophiuchus, where the $160 \mu \mathrm{m}$ maps are saturated in all of the dense source regions. Even for isolated sources, the measured $160 \mu \mathrm{m}$ flux density, determined from a point-spread function (PSF) fit, may be underestimated if the source is extended. The effects of these issues on our analysis are discussed in more detail in the Appendix.

\subsection{Identifying Embedded Protostar Candidates}

We form a sample of candidate embedded protostars from the c2d catalogs; the first cut is based on the source "class." All sources in the c2d catalogs are assigned a class parameter based on colors, magnitudes, and stellar SED fits (see the c2d Delivery Document (Evans et al. 2007, and Harvey et al. 2007a). Class parameters include "star," "star+disk," "YSOc" (YSO candidate), "red," "rising," "Galc" (galaxy candidate), etc. Embedded protostars will generally be a subset of YSOc sources, but some of the most embedded may also be assigned to the "red" class if they are not detected in all IRAC bands.

Thus, we begin by selecting all sources from the c2d database that are classified as "YSOc" or "red." From this list, we keep sources that meet all of the following criteria: (a) flux density at $24 \mu \mathrm{m}\left(S_{24 \mu \mathrm{m}}\right) \geqslant 3 \mathrm{mJy}$. (b) $S_{24 \mu \mathrm{m}} \geqslant 5 \alpha_{\mathrm{IR}}+8 \mathrm{mJy}$, where $\alpha_{\mathrm{IR}}$ is the near- to mid-IR spectral index, determined by a leastsquares fit to photometry between 2 and $24 \mu \mathrm{m}$. This criterion is motivated by a comparison to the carefully vetted Serpens YSO sample of Harvey et al. (2007a). (c) $v S_{24 \mu \mathrm{m}}>v S_{8 \mu \mathrm{m}}$, i.e., the SED is rising from 8 to $24 \mu \mathrm{m}$ in $\nu S_{\nu}$ space. (d) $S_{24} \mu \mathrm{m}$ must be of high quality, i.e., signal to noise $(\mathrm{S} / \mathrm{N})$ greater than 7 . (e) $S_{24 \mu \mathrm{m}}$ is not a "band-filled" flux. For sources not originally detected in all Spitzer bands, a flux or upper limit is measured at the source position (band-filling; Harvey et al. 2007a). Because the resolution is lower at $24 \mu \mathrm{m}$ than at the shorter wavelengths, some IRAC-only sources have unreliable band-filled fluxes at $24 \mu \mathrm{m}$ (e.g., sources are confused with the PSF wings of a nearby source).

In addition to sources that meet the above criteria, we include any $70 \mu \mathrm{m}$ point sources not classified as galaxy candidates 


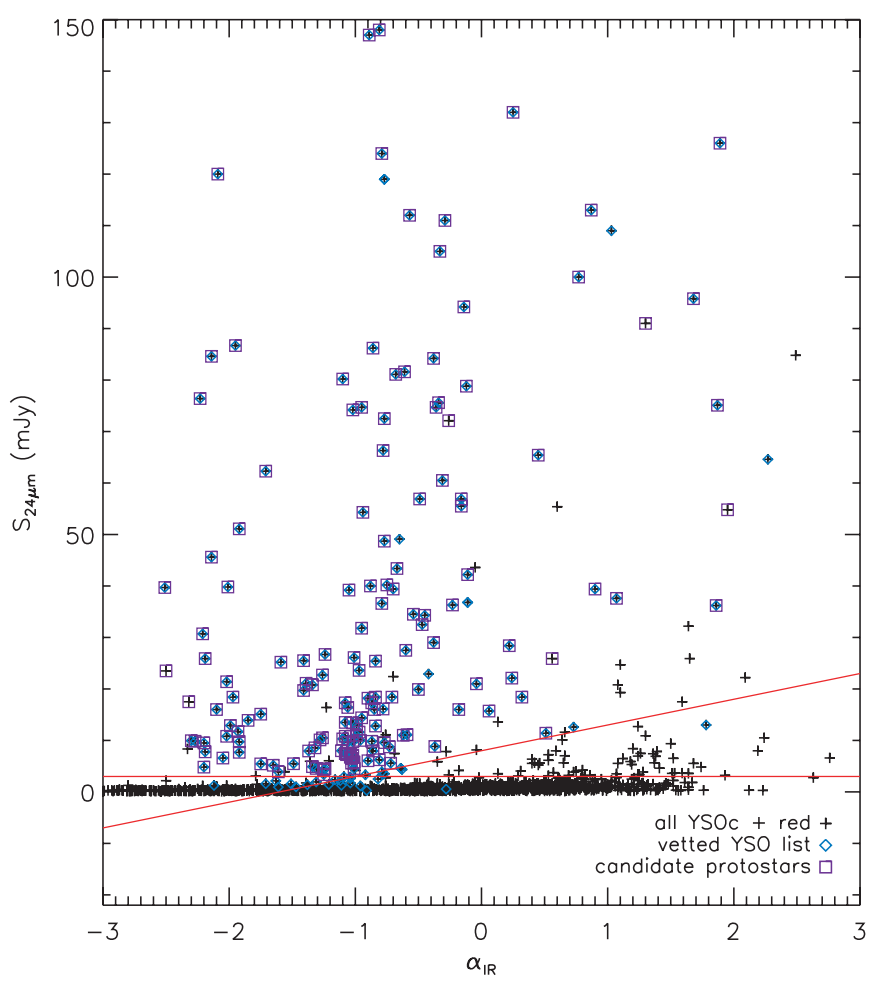

Figure 2. Plot of $S_{24 \mu \mathrm{m}}$ vs. spectral index $\alpha_{\mathrm{IR}}$ for sources in Serpens, to demonstrate selection criteria for the embedded protostar candidate samples. The plus symbols ("+") indicate the sample from which the candidate embedded protostars are drawn, including all catalog sources labeled as "YSOc" or "red" as well as non-"Galc" $70 \mu \mathrm{m}$ sources. Lines show $24 \mu \mathrm{m}$ flux cuts imposed by criteria (a) and (b), which remove the majority of spurious or background sources. Boxes indicate the final embedded protostar sample, after applying criteria (a)-(d). Smaller blue diamonds show the carefully vetted YSO sample from Harvey et al. (2006). The two samples agree fairly well, but because we select for embedded sources we miss many of the more evolved YSOs in the vetted YSO list (Class II/III). There are a few sources in the embedded protostar sample not in the Harvey et al. (2006) list, two of which are likely to be real (see the text), while the others are rejected when examined by eye.

(A color version of this figure is available in the online journal.)

("Galc"). Note that these $70 \mu \mathrm{m}$ sources need not be classified as "red" or "YSOc." In each cloud, a number of deeply embedded sources that are bright at $70 \mu \mathrm{m}$ but very weak at $24 \mu \mathrm{m}$ (e.g., $\mathrm{HH} 211$ in Perseus) are recovered by this last criterion (five in Perseus, four in Serpens, and three in Ophiuchus), as are a few very bright sources that are saturated at $24 \mu \mathrm{m}$ (six in Perseus, two in Serpens, and six in Ophiuchus; these are often classified as "rising").

Figure 2 plots $S_{24 \mu \mathrm{m}}$ versus $\alpha_{\mathrm{IR}}$ for embedded candidates in Serpens, where "+" symbols indicate the original ("YSOc"+"red"+ $70 \mu \mathrm{m}$ sources) sample for Serpens, and boxes indicate our embedded protostar candidates after applying criteria (a)-(e). For comparison, diamonds indicate the carefully vetted Serpens YSO list from Harvey et al. (2007a). The majority of "+"-only points, which were rejected as true YSOs by Harvey et al. (2007a), are removed by criteria (a) and (b) (shown as solid lines). More than half of the vetted YSOs do not appear in the candidate embedded protostar sample (diamond, no box); the majority of these are rejected by the rising SED criteria (c) and as they are primarily classified as "star+disk" we do not expected them to be embedded. There are a few sources in our sample that are not in the vetted YSO sample (box, no diamond). A few of these were easily identified as nonprotostellar, and rejected, when examining by eye. We do identify three embedded protostar candidates in Serpens that are not in the

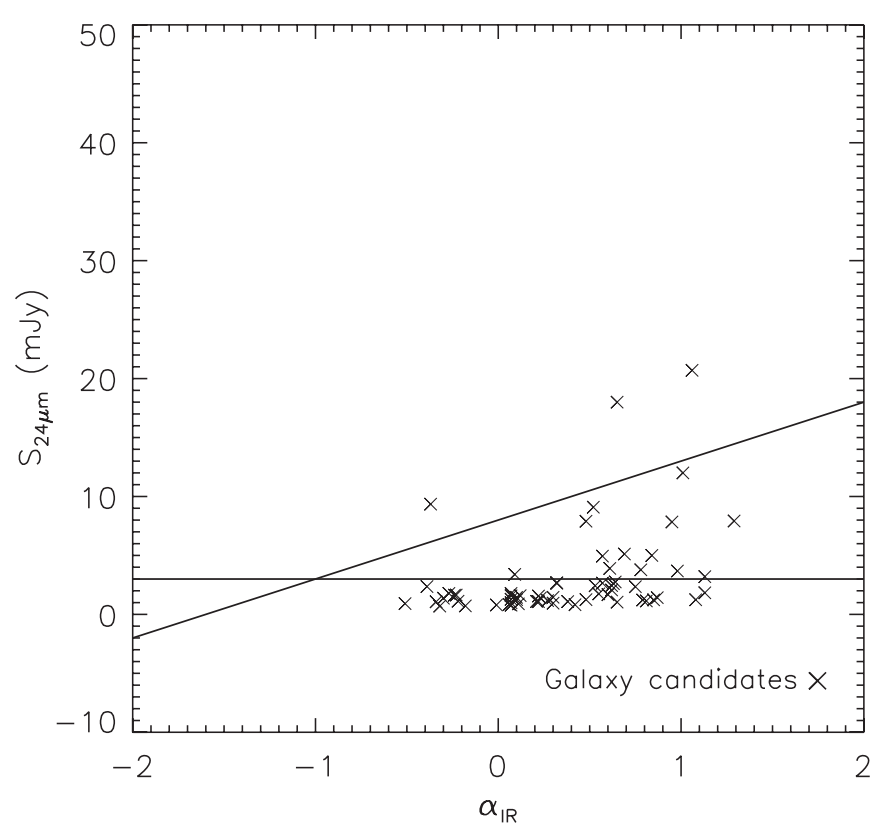

Figure 3. Plot of $S_{24} \mu \mathrm{m}$ vs. spectral index $\alpha_{\mathrm{IR}}$ for galaxy candidates ("Galc") in Serpens. Selection criteria (a) and (b) are overlaid, as in Figure 2. These selection criteria eliminate most of the parameter space inhabited by extragalactic sources.

vetted YSO list but seem to be associated with $1.1 \mathrm{~mm}$ emission (see Section 2.): one "red" and two "rising."

We include red sources to ensure that we identify the most embedded protostars, but we need to reject the large percentage of these sources that are likely to be galaxies. As shown in Figure 3, criteria (a) and (b) are efficient at eliminating extragalactic sources. Here, $S_{24 \mu \mathrm{m}}$ is plotted versus $\alpha_{\mathrm{IR}}$ for all "Galc" galaxy candidates in Serpens. As in Figure 2, lines show criteria (a) and (b).

After forming our embedded candidate samples based on the above criteria, the images and SEDs of each source are examined by eye to remove any galaxies that are extended in the near-IR, and other obviously nonembedded sources. SEDs include 2MASS, IRAC, MIPS, and $1.1 \mathrm{~mm}$ fluxes. In some cases, there is no available point-source flux at 70 or $160 \mu \mathrm{m}$ even if there is emission at the position of the source, generally because the source is extended at these wavelengths. In these cases a flux density is measured by hand, if possible, using aperture photometry. Large uncertainties $(50 \%$ or more) are associated with these band-filled flux measurements.

\subsection{Association with a $1.1 \mathrm{~mm}$ Core}

The next step after assembling a sample of candidate embedded protostars is to determine which are associated with $1.1 \mathrm{~mm}$ emission. The correlation between candidate protostar and $1.1 \mathrm{~mm}$ core positions was done in Enoch et al. (2008, see Section 2.3 and Figure 2), following a similar analysis by Jørgensen et al. (2007); we summarize the results here. We found that the coldest protostellar candidates $\left(T_{\mathrm{bol}}<300 \mathrm{~K}\right.$, see Section 3.1$)$ are primarily located within $1.0 \times \theta_{1 \mathrm{~mm}}$ of a millimeter core position, where $\theta_{1 \mathrm{~mm}}$ is the angular FWHM size of the $1.1 \mathrm{~mm}$ core, as expected if they are deeply embedded. Based on that analysis, a given embedded protostar candidate is assumed to be associated with a millimeter core if it is located within $1.0 \times \theta_{1 \mathrm{~mm}}$ of the $1.1 \mathrm{~mm}$ core centroid position.

We also found in Enoch et al. (2008), based on analysis of a spatially random distribution of sources, that we can expect 
approximately five false associations with $1.1 \mathrm{~mm}$ cores in each cloud. Using a more restrictive criteria (e.g., $0.5 \times \theta_{1 \mathrm{~mm}}$ ) would reduce the number of false associations, but would likely miss at least a few embedded protostars. For reference, adopting $0.5 \times \theta_{1 \mathrm{~mm}}$ would result in 10 fewer embedded protostars in Perseus, 7 in Serpens, and 16 in Ophiuchus.

If an embedded protostar candidate is found to be associated with a millimeter core by the above criteria, the $1.1 \mathrm{~mm}$ flux is included in the protostellar SED, and is used to calculate an envelope mass ( $M_{\mathrm{env}}$, Section 3.2). If a candidate is not located within $1.0 \times \theta_{1 \mathrm{~mm}}$ of a millimeter core position, we calculate a flux or upper limit from the original $1.1 \mathrm{~mm}$ map (although sources with upper limits at $1.1 \mathrm{~mm}$ are not included in the final lists of embedded protostars given in Tables 2-4).

In a number of cases (10 in Perseus, 3 in Serpens, and 11 in Ophiuchus) there is clearly $1.1 \mathrm{~mm}$ flux at the protostar position that was not identified as a core in the original $1.1 \mathrm{~mm}$ source extraction because it is below $5 \sigma$ (where $\sigma$ is the local rootmean-square (rms) noise, see Paper I) or is in a confused region of the map. If this emission exceeds $3 \sigma$, we measure a "bandfilled" $1.1 \mathrm{~mm}$ flux using a small aperture $\left(30^{\prime \prime}-40^{\prime \prime}\right)$. We also re-compute the $1.1 \mathrm{~mm}$ flux density in small apertures for all sources in regions of blended $1.1 \mathrm{~mm}$ emission. When more than one protostar candidate is associated with a single compact millimeter core, we divide the total $1.1 \mathrm{~mm}$ flux of the core equally between the protostellar sources.

The approach described above means that the $1.1 \mathrm{~mm}$ flux is not measured in a fixed aperture for every source. In crowded regions or for sources with close neighbors, the $1.1 \mathrm{~mm}$ flux is measured in a $30^{\prime \prime}-40^{\prime \prime}$ diameter aperture centered on the Spitzer source position. These sources are indicated by a footnote in Tables 2-4. For other sources, the Bolocam $1.1 \mathrm{~mm}$ core flux from Papers I-III is used. Typically, but not always, the total core flux is used here (integrated in the largest aperture, from $30^{\prime \prime}$ to $120^{\prime \prime}$ diameters in steps of $10^{\prime \prime}$, that is smaller than the distance to the nearest neighboring source); fluxes in 30, 40, 80, and $120^{\prime \prime}$ apertures can also be found in Papers I-III.

Approximately $50 \%$ of the embedded protostar candidates in each cloud are lacking $1.1 \mathrm{~mm}$ emission, even after reexamining the $1.1 \mathrm{~mm}$ maps at each source position. Most of these sources appear to be Class II objects, with little or no remaining envelope. Any $1.1 \mathrm{~mm}$ emission from these objects, therefore, is likely below our detection limit of $\sim 0.1 M_{\odot}$ (Section 3.3). A few of these may actually be low-luminosity sources that are truly embedded, with low-mass envelopes below our detection limit. For this reason, we only claim completeness to embedded protostars with $M_{\text {env }}>0.1 M_{\odot}$. Note that this corresponds well to the Crapsi et al. (2008) definition of Stage I.

\section{PROPERTIES OF EMBEDDED PROTOSTARS}

\subsection{Bolometric Luminosity and Temperature}

Our extensive wavelength coverage allows us to construct well-sampled SEDs for the embedded protostar candidates in all three clouds, from which we calculate a bolometric luminosity $\left(L_{\text {bol }}\right)$ and bolometric temperature $\left(T_{\text {bol }}\right)$ for each source. The bolometric luminosity is calculated by integrating the $\operatorname{SED}\left(S_{v}\right)$ over frequency:

$$
L_{\mathrm{bol}}=4 \pi d^{2} \int S_{\nu} d \nu
$$

The bolometric temperature is defined as the temperature of a blackbody with the same mean frequency as the source SED, and is given by

$$
T_{\mathrm{bol}}=1.25 \times 10^{-11}\langle\nu\rangle \mathrm{K}
$$

(Myers \& Ladd 1993), where the mean frequency is

$$
\langle v\rangle=\frac{\int v S_{v} d v}{\int S_{v} d v} .
$$

Two methods for approximating the integrations over frequency for finitely sampled SEDs (midpoint and prismoidal) are discussed in the Appendix.

Tables 2-4 list the bolometric temperatures and luminosities derived for all embedded protostars in each cloud. As we are primarily interested in young, embedded objects, only sources with detectable $1.1 \mathrm{~mm}$ emission, which likely have substantial envelopes, are included. Sources are listed by increasing $T_{\mathrm{bol}}$, and identified as, e.g., "Per-emb\#," as well as by their c2d name (SSTc2dJ...), which also gives the position. When sources are saturated at 24 or $70 \mu \mathrm{m}$, the IRAS 25 or $60 \mu \mathrm{m}$ flux is utilized when not affected by blending. In these cases, a note is made in Tables 2-4. The correction of saturated fluxes can increase the luminosity by more than a factor of 2 ; for saturated sources with no reliable IRAS flux (also noted in Tables 2-4), the luminosity will be an underestimate.

All $T_{\text {bol }}$ and $L_{\text {bol }}$ values quoted use the midpoint integration method. The difference between the values calculated by the midpoint and prismoidal integration methods (given in parentheses in Tables 2-4) gives a more realistic measure of the uncertainties in $T_{\mathrm{bol}}$ and $L_{\mathrm{bol}}$ than the formal fitting errors (which are typically $10 \%$ ). Uncertainties can be larger than $50 \%$ depending on whether or not a $160 \mu \mathrm{m}$ flux is available, and there is an additional systematic uncertainty of $15 \%-25 \%$ from finite sampling errors (see the Appendix).

Systematic errors introduced by missing $160 \mu \mathrm{m}$ fluxes are investigated in the Appendix. Without a $160 \mu \mathrm{m}$ measurement, $T_{\text {bol }}$ will almost certainly be an overestimate for very cold sources, which may bias our classification of protostellar candidates (Section 4). Ophiuchus will be most affected, as only four sources in that cloud have reliable $160 \mu \mathrm{m}$ fluxes.

\subsubsection{Correcting for Extinction}

One might argue that we should correct our photometry for extinction before calculating $L_{\mathrm{bol}}$ and $T_{\mathrm{bol}}$. The effects of extinction are typically ignored for Class 0 and Class I sources, for which one might expect the foreground extinction to be negligible relative to the effect of the envelope itself. While these observed values are most easily compared to the majority of previous work, Chen et al. (1995), who connected $T_{\text {bol }}$ to the classes defined by $\alpha$, did correct the observed flux densities for extinction before computing $T_{\mathrm{bol}}$. Therefore, we compute extinction-corrected values for comparison (see Evans et al. 2009 for a more complete discussion of the effects of dereddening). We use the mean extinction to all Class II sources in each cloud $\left(A_{V}=5.92 \mathrm{mag}\right.$ in Perseus, $9.57 \mathrm{mag}$ in Serpens, 9.76 mag in Ophiuchus; Evans et al. 2009) to deredden the photometry of Class 0 and Class I sources.

As dereddening has a greater effect on shorter wavelength photometry, correcting for extinction tends to increase both $T_{\text {bol }}$ and $L_{\text {bol }}$. Throughout this paper we will primarily use observed values, and not extinction-corrected values, but the 
Table 1

Definition of Classes and Stages

\begin{tabular}{lc}
\hline \hline Class/Stage & Definition \\
\hline Class 0 & $T_{\text {bol }} \leqslant 70 \mathrm{~K}$ \\
Class I & $70 \mathrm{~K}<T_{\text {bol }} \leqslant 650 \mathrm{~K} ; 1.1 \mathrm{~mm}$ detection $\left(M_{\text {env }} \gtrsim 0.1 M_{\odot}\right)$ \\
Class II & $650 \mathrm{~K}<T_{\text {bol }} \leqslant 2800 \mathrm{~K}$ \\
\hline Early Class 0 & $T_{\text {bol }} \leqslant 50 \mathrm{~K}$ \\
Late Class 0 & $50 \mathrm{~K}<T_{\text {bol }} \leqslant 100 \mathrm{~K}$ \\
Early Class I & $100 \mathrm{~K}<T_{\text {bol }} \leqslant 300 \mathrm{~K}$ \\
Late Class I & $300 \mathrm{~K}<T_{\text {bol }} \leqslant 650 \mathrm{~K}$ \\
Nonenvelope Class I & $70 \mathrm{~K}<T_{\text {bol }} \leqslant 650 \mathrm{~K} ; n o 1.1$ mm detection $\left(M_{\text {env }} \lesssim 0.1 M_{\odot}\right)$ \\
\hline Stage 0 & $M_{*}<M_{\text {env }}$ \\
Stage I & $M_{*}>M_{\text {env }} ; M_{\text {env }} \geqslant 0.1 M_{\odot}$ \\
Stage II & Circumstellar disk; $M_{\text {env }}<0.1 M_{\odot}$
\end{tabular}

effects on derived values such as the Class 0 lifetime (Section 9) will be noted. When used, the extinction-corrected bolometric luminosity and temperature are indicated by $T_{\text {bol }}^{\prime}$ and $L_{\text {bol }}^{\prime}$, respectively.

\subsection{Envelope Mass}

The envelope mass of candidate protostars, $M_{\mathrm{env}}$, is calculated from the flux density at $1.1 \mathrm{~mm}, S_{1 \mathrm{~mm}}$ :

$$
M=\frac{d^{2} S_{1 \mathrm{~mm}}}{B_{1 \mathrm{~mm}}\left(T_{D}\right) \kappa_{1 \mathrm{~mm}}},
$$

where $d$ is the cloud distance, $\kappa_{1 \mathrm{~mm}}=0.0114 \mathrm{~cm}^{2} \mathrm{~g}^{-1}$ is the dust opacity per gram of gas at $1.1 \mathrm{~mm}$, and $B_{1 \mathrm{~mm}}$ is the Planck function at a dust temperature of $T_{D}$. The opacity is interpolated from Table 1 (Column 5) of Ossenkopf \& Henning (1994) for dust grains with thin ice mantles.

We assume a dust temperature of $T_{D}=15 \mathrm{~K}$ for protostellar envelopes, consistent with average isothermal dust temperatures found from radiative transfer models of a sample of Class 0 and Class I protostars (Shirley et al. 2002; Young et al. 2003). The isothermal dust temperature is the temperature that, when used in an isothermal mass equation (e.g., Equation (4)), yields the same mass as does a detailed radiative transfer model that accounts for temperature gradients. Dust temperatures will be higher than $15 \mathrm{~K}$ close to the protostar, but most of the envelope mass is in the outer, cooler, regions of the envelope. A dust temperature of $10 \mathrm{~K}$ would result in an increase in masses by a factor of 1.9 , while $T_{D}=20 \mathrm{~K}$ would decrease masses by a factor of 1.5.

Envelope masses of embedded protostars are listed in Tables 2-4. If the source is associated with a distinct core, the Bolocam identification from Papers I-III is given in the last column. If the source is not associated with one of the originally identified cores, but rather the flux has been "band-filled" at 1.1 $\mathrm{mm}$, then no Bolocam ID is given. In these "band-filled" cases, the measured $1.1 \mathrm{~mm}$ flux can be easily re-constructed from the mass.

\subsection{Completeness}

Because we require a detection at $1.1 \mathrm{~mm}$ to be included in the final source lists, we are clearly incomplete to objects more evolved than Stage I, which do not have a substantial protostellar envelope. We do detect a few Class II sources at $1.1 \mathrm{~mm}$; these are discussed in Section 5.1. The point-source detection limits of our $1.1 \mathrm{~mm}$ surveys limit our sensitivity to Class I sources with $M_{\text {env }} \geq 0.09 M_{\odot}$ in Perseus, $M_{\text {env }} \geq 0.07 M_{\odot}$ in Serpens, and $M_{\text {env }} \gtrsim 0.04 M_{\odot}$ in Ophiuchus. These are $5 \sigma$ point source detection limits; for very extended envelopes the completeness limits will be higher. For simplicity, we take a detection limit of $0.1 M_{\odot}$ for all clouds.

As the $1.1 \mathrm{~mm}$ detection requirement is more restrictive than our $24 \mu \mathrm{m}$ flux criteria, we explore the possibility that we are missing some low-luminosity embedded sources that are below our $1.1 \mathrm{~mm}$ detection threshold.

Taking the $1.1 \mathrm{~mm} 5 \sigma$ detection limits for each cloud (75 mJy in Perseus, $50 \mathrm{mJy}$ in Serpens, and $110 \mathrm{mJy}$ in Ophiuchus), and assuming the spectrum of a modified blackbody, $S_{v}=v^{\beta} B_{v}\left(T_{D}\right)$, with $T_{D}=15 \mathrm{~K}$, we can estimate the minimum detectable bolometric luminosity in each cloud. For $\beta=1$, the minimum $L_{\text {bol }}$ is $0.02 L_{\odot}$ in Perseus, $0.01 L_{\odot}$ in Serpens, and $0.01 L_{\odot}$ in Ophiuchus. Assuming $T_{D}=20 \mathrm{~K}$ lowers these values by approximately a factor of 2 , while taking $\beta=2$ increases them by a factor of 4 . Although these are very rough estimates, they agree fairly well with the lowest observed bolometric luminosities for sources with $1.1 \mathrm{~mm}$ detections $\left(0.04 L_{\odot}\right.$ in Perseus, $0.05 L_{\odot}$ in Serpens, and $0.01 L_{\odot}$ in Ophiuchus). Dunham et al. (2008) and Harvey et al. (2007b) demonstrate that the Spitzer c2d surveys are complete to young objects with luminosities as low as $0.05 L_{\odot}$.

Finally, a comparison of our source list to the dedicated search for very low-luminosity protostars by Dunham et al. (2008) confirms that we are not missing any embedded protostellar sources in Perseus down to the completeness limits of that survey $\left(L_{\text {bol }} \gtrsim 0.03-0.05 L_{\odot}\right)$.

\section{SOURCE CLASSIFICATION}

To study the early evolution of protostars, it is necessary to identify the evolutionary state of the embedded candidates in our sample. This is typically accomplished by classifying sources into discrete groups based on SED characteristics. A number of classification methods are employed in the literature; most often used are the near- to mid-IR spectral index $\alpha_{\mathrm{IR}}=$ $d \log \left(\lambda F_{\lambda}\right) / d \log (\lambda)$ (Lada 1987), the bolometric temperature $T_{\text {bol }}$ (Myers \& Ladd 1993, see Section 3.1), and the ratio of submillimeter to bolometric luminosity $L_{\text {submm }} / L_{\text {bol }}$ (André et al. 1993). Generally, $L_{\text {submm }}$ is taken to be the integrated luminosity at wavelengths $\lambda \geqslant 350 \mu \mathrm{m}$.

Any of these methods must come with the caveat that protostellar mass may affect the classification, which only detailed modeling can resolve (e.g., Hatchell et al. 2007b). We typically have only one flux measurement for $\lambda>160 \mu \mathrm{m}$, so 
Table 2

Bolometric Temperatures, Luminosities, and Envelope Masses of Embedded Protostars in Perseus

\begin{tabular}{|c|c|c|c|c|c|c|c|}
\hline ID & $\begin{array}{c}\text { c2d Name/Position } \\
(\mathrm{SSTc} 2 \mathrm{dJ} . . .)\end{array}$ & $\begin{array}{l}T_{\text {bol }} \\
(\mathrm{K}) \\
\end{array}$ & $\begin{array}{l}L_{\text {bol }} \\
\left(L_{\odot}\right)\end{array}$ & $\alpha_{\mathrm{IR}}$ & $\begin{array}{l}M_{\text {env }} \\
\left(M_{\odot}\right)\end{array}$ & Bolocam ID & Other Names \\
\hline \multicolumn{8}{|c|}{ Class 0} \\
\hline Per-emb 1 & J034356.53+320052.9 & $24(5)$ & $1.5(0.4)$ & $0.5(0.18)$ & $3.02(0.08)$ & Bolo 103 & HH 211 \\
\hline Per-emb 2 & J033217.95+304947.6 & $25(2)$ & $1.3(0.2)$ & $1.07(0.11)$ & $2.88(0.08)$ & Bolo 66 & IRAS $03292+3039$ \\
\hline Per-emb 3 & J032900.52+311200.7 & $30(4)$ & $0.69(0.15)$ & $2.16(0.1)$ & $0.29(0.03)$ & Bolo 41 & \\
\hline Per-emb 4 & J032839.10+310601.8 & $31(3)$ & $0.22(0.03)$ & $1.68(0.06)$ & $0.36(0.02)$ & Bolo 30 & \\
\hline Per-emb 5 & J033120.96+304530.2 & $33(1)$ & $1.2(0.2)$ & $0.98(0.06)$ & $0.95(0.04)$ & Bolo 65 & IRAS $03282+3035$ \\
\hline Per-emb 6 & $\mathrm{~J} 033314.40+310710.9$ & $36(5)$ & $0.64(0.01)$ & $2.22(0.06)$ & $1.04(0.1)$ & Bolo $79^{a}$ & \\
\hline Per-emb 7 & J033032.68+302626.5 & $37(4)$ & $0.15(0.06)$ & $2.08(0.07)$ & $0.99(0.08)$ & Bolo 62 & \\
\hline Per-emb 8 & J034443.94+320136.1 & $40(6)$ & $3.0(0.5)$ & $0.96(0.07)$ & $0.63(0.06)$ & Bolo $116^{\mathrm{a}}$ & \\
\hline Per-emb 9 & J032951.82+313906.1 & $41(2)$ & $0.46(0.01)$ & $3.44(0.11)$ & $0.64(0.04)$ & Bolo 59 & IRAS $03267+3128$ \\
\hline Per-emb 10 & J033316.45+310652.5 & $47(14)$ & $0.27(0.66)$ & $1.73(0.09)$ & $1.57(0.16)$ & Bolo $79^{a}$ & \\
\hline Per-emb 11 & J034356.85+320304.6 & $49(15)$ & $0.45(0.85)$ & $1.37(0.09)$ & $1.78(0.04)$ & Bolo 104 & IC 348-MMS \\
\hline Per-emb 12 & $\mathrm{~J} 032910.50+311331.0$ & $51(17)$ & $4.2(4.4)$ & $2.58(0.08)$ & $7.75(0.78)$ & Bolo $48^{\mathrm{a}}$ & NGC 1333-IRAS 4A \\
\hline Per-emb 13 & J032912.04+311301.5 & $54(18)$ & $1.6(2.0)$ & $0.55(0.06)$ & $3.66(0.37)$ & Bolo $48^{\text {a }}$ & NGC 1333-IRAS 4B \\
\hline Per-emb 14 & J032913.52+311358.0 & $54(14)$ & $0.49(0.32)$ & $2.41(0.13)$ & $0.5(0.05)$ & Bolo $48^{\mathrm{a}}$ & NGC 1333-IRAS 4C \\
\hline Per-emb 15 & J032904.05+311446.6 & $56(16)$ & $0.53(0.67)$ & $1.43(0.06)$ & $1.16(0.12)$ & Bolo 46 & \\
\hline Per-emb 16 & J034350.95+320324.8 & $56(17)$ & $0.38(0.62)$ & $1.51(0.06)$ & $1.15(0.11)$ & Bolo $102^{\mathrm{a}}$ & \\
\hline Per-emb 17 & J032739.09+301303.0 & $59(11)$ & $4.2(0.1)$ & $2.68(0.06)$ & $0.51(0.03)$ & Bolo 22 & RNO 15-FIR \\
\hline Per-emb 18 & J032911.25+311831.3 & $59(12)$ & $2.8(1.7)$ & $1.94(0.06)$ & $2.47(0.07)$ & Bolo $49^{\mathrm{b}}$ & \\
\hline Per-emb 19 & J032923.49+313329.5 & $60(3)$ & $0.36(0.05)$ & $1.51(0.06)$ & $0.64(0.05)$ & Bolo 57 & \\
\hline Per-emb 20 & $\mathrm{~J} 032743.23+301228.8$ & $60(14)$ & $1.7(0.01)$ & $2.39(0.06)$ & $0.5(0.03)$ & Bolo 23 & L1455-IRS 4 \\
\hline Per-emb 21 & J032910.68+311820.5 & $63(12)$ & $2.8(1.9)$ & $1.95(0.06)$ & $2.47(0.07)$ & Bolo $49^{b}$ & \\
\hline Per-emb 22 & $\mathrm{~J} 032522.33+304514.0$ & $63(11)$ & $1.7(1.1)$ & $2.34(0.07)$ & $1.41(0.14)$ & Bolo 5 & L 1448-IRS2 \\
\hline Per-emb 23 & J032917.16+312746.4 & $66(18)$ & $0.29(0.33)$ & $1.75(0.06)$ & $0.5(0.05)$ & Bolo 52 & \\
\hline Per-emb 24 & $\mathrm{~J} 032845.30+310542.0$ & $67(10)$ & $0.43(0.01)$ & $1.11(0.05)$ & $0.19(0.02)$ & Bolo $33^{\mathrm{a}}$ & \\
\hline Per-emb 25 & J032637.46+301528.0 & $68(12)$ & $0.95(0.02)$ & $1.09(0.05)$ & $0.5(0.08)$ & Bolo 18 & \\
\hline Per-emb 26 & J032538.82+304406.3 & $69(7)$ & $4.4(1.5)$ & $2.16(0.06)$ & $1.87(0.19)$ & Bolo $10^{\mathrm{a}}$ & L 1448-C \\
\hline Per-emb $27^{\mathrm{c}}$ & J032855.56+311436.6 & $69(1)$ & $19.0(0.4)$ & $3.03(0.12)$ & $2.81(0.28)$ & Bolo $38^{\mathrm{a}}$ & NGC 1333-IRAS 2A \\
\hline \multicolumn{8}{|c|}{ Class I } \\
\hline Per-emb 28 & J034351.02+320307.9 & $72(25)$ & $0.28(0.37)$ & $-0.28(0.06)$ & $0.62(0.06)$ & Bolo $102^{\mathrm{a}}$ & \\
\hline Per-emb 29 & J033317.85+310932.0 & $76(20)$ & $1.8(2.5)$ & $3.33(0.06)$ & $3.84(0.09)$ & Bolo 80 & $\mathrm{~B} 1-\mathrm{c}$ \\
\hline Per-emb 30 & J033327.28+310710.2 & $78(6)$ & $1.7(0.01)$ & $1.93(0.05)$ & $0.59(0.06)$ & Bolo 84 & \\
\hline Per-emb 31 & $\mathrm{~J} 032832.55+311105.2$ & $80(13)$ & $0.16(0.01)$ & $0.78(0.05)$ & $0.19(0.02)$ & Bolo 25 & \\
\hline Per-emb 32 & J034402.40+320204.9 & $84(29)$ & $0.16(0.31)$ & $1.53(0.06)$ & $0.63(0.02)$ & Bolo 106 & \\
\hline Per-emb 33 & J032536.48+304522.3 & $90(18)$ & $4.3(3.7)$ & $2.62(0.07)$ & $4.47(0.45)$ & Bolo 8 & L $1448-\mathrm{N}$ \\
\hline Per-emb 34 & J033015.12+302349.2 & $99(13)$ & $1.6(0.1)$ & $1.7(0.05)$ & $0.46(0.07)$ & Bolo 60 & IRAS $03271+3013$ \\
\hline Per-emb 35 & J032837.09+311330.7 & $103(26)$ & $9.1(0.3)$ & $2.35(0.08)$ & $0.44(0.04)$ & Bolo 29 & NGC 1333-IRAS 1 \\
\hline Per-emb 36 & J032857.36+311415.7 & $106(12)$ & $5.3(1.0)$ & $1.6(0.06)$ & $0.74(0.07)$ & Bolo $38^{\mathrm{a}}$ & \\
\hline Per-emb 37 & J032918.27+312320.0 & $106(21)$ & $0.07(0.35)$ & $1.26(0.06)$ & $0.44(0.04)$ & Bolo $54^{\mathrm{a}}$ & \\
\hline Per-emb 38 & J033229.18+310240.9 & $115(21)$ & $0.54(0.01)$ & $0.4(0.05)$ & $0.45(0.08)$ & Bolo 68 & \\
\hline Per-emb 39 & $\mathrm{~J} 033313.78+312005.2$ & $125(47)$ & $0.04(0.08)$ & $1.44(0.06)$ & $0.41(0.04)$ & Bolo 78 & \\
\hline Per-emb 40 & J033316.66+310755.2 & $132(25)$ & $1.5(1.0)$ & $1.57(0.05)$ & $1.12(0.11)$ & & $\mathrm{B} 1-\mathrm{a}$ \\
\hline Per-emb 41 & J033320.30+310721.3 & $157(72)$ & $0.17(0.36)$ & $0.88(0.05)$ & $1.5(0.15)$ & Bolo 81 & $\mathrm{~B} 1-\mathrm{b}$ \\
\hline Per-emb 42 & $\mathrm{~J} 032539.10+304358.0$ & $163(51)$ & $0.68(0.85)$ & $2.36(0.06)$ & $1.82(0.18)$ & Bolo $10^{\mathrm{a}}$ & \\
\hline Per-emb 43 & J034202.16+314802.1 & $176(42)$ & $0.07(0.06)$ & $1.47(0.05)$ & $0.07(0.02)$ & & \\
\hline Per-emb $44^{\mathrm{c}}$ & J032903.76+311603.7 & $188(9)$ & $32.5(7.1)$ & $1.21(0.08)$ & $3.25(0.32)$ & Bolo 43 & SVS 13A \\
\hline Per-emb 45 & J033309.57+310531.2 & $197(93)$ & $0.05(0.06)$ & $1.13(0.05)$ & $0.21(0.02)$ & & \\
\hline Per-emb 46 & J032800.40+300801.3 & $221(7)$ & $0.3(0.07)$ & $0.95(0.05)$ & $0.17(0.02)$ & & \\
\hline Per-emb 47 & $\mathrm{~J} 032834.50+310051.1$ & $230(17)$ & $1.2(0.1)$ & $0.83(0.05)$ & $0.12(0.02)$ & & IRAS $03254+3050$ \\
\hline Per-emb 48 & J032738.23+301358.8 & $238(14)$ & $0.87(0.04)$ & $-0.19(0.05)$ & $0.34(0.04)$ & Bolo 21 & L1455 FIR2 \\
\hline Per-emb 49 & J032912.94+311814.4 & $239(68)$ & $1.1(0.7)$ & $1.05(0.05)$ & $0.77(0.08)$ & Bolo $49^{\mathrm{a}}$ & ASR 30 \\
\hline Per-emb $50^{d}$ & J032907.76+312157.2 & $254(23)$ & $10.0(3.0)$ & $2.18(0.1)$ & $1.62(0.16)$ & Bolo 47 & \\
\hline Per-emb 51 & $\mathrm{~J} 032834.53+310705.5$ & $263(115)$ & $0.07(0.1)$ & $0.54(0.05)$ & $0.24(0.04)$ & Bolo 28 & \\
\hline Per-emb 52 & J032839.72+311731.9 & $278(119)$ & $0.16(0.21)$ & $0.57(0.05)$ & $0.49(0.05)$ & Bolo $31^{\mathrm{a}}$ & \\
\hline Per-emb 53 & J034741.56+325143.9 & $287(8)$ & $4.7(0.9)$ & $0.78(0.05)$ & $1.67(0.08)$ & Bolo 122 & B5-IRS1 \\
\hline Per-emb $54^{\mathrm{d}}$ & J032901.57+312020.7 & $304(63)$ & $6.2(2.6)$ & $2.09(0.08)$ & $1.87(0.19)$ & Bolo 42 & NGC 1333-IRAS 6 \\
\hline Per-emb 55 & J034443.33+320131.4 & $309(64)$ & $1.8(0.8)$ & $0.5(0.05)$ & $0.32(0.03)$ & Bolo $116^{\mathrm{a}}$ & IRAS $03415+3152$ \\
\hline Per-emb 56 & J034705.42+324308.4 & $312(1)$ & $0.54(0.09)$ & $0.36(0.05)$ & $0.14(0.02)$ & & IRAS $03439+3233$ \\
\hline Per-emb 57 & J032903.33+312314.6 & $313(200)$ & $0.09(0.45)$ & $1.13(0.05)$ & $0.11(0.02)$ & & \\
\hline Per-emb 58 & J032858.44+312217.4 & $322(88)$ & $0.63(0.47)$ & $0.83(0.05)$ & $0.71(0.07)$ & Bolo 40 & \\
\hline Per-emb 59 & J032835.04+302009.9 & $341(179)$ & $0.04(0.06)$ & $0.15(0.05)$ & $0.14(0.02)$ & Bolo 27 & \\
\hline Per-emb 60 & J032920.07+312407.5 & $363(240)$ & $0.28(1.05)$ & $0.42(0.05)$ & $0.52(0.05)$ & Bolo $54^{\mathrm{a}}$ & \\
\hline Per-emb 61 & J034421.33+315932.6 & $371(107)$ & $0.24(0.16)$ & $0.21(0.05)$ & $0.17(0.02)$ & Bolo 113 & \\
\hline Per-emb 62 & $\mathrm{~J} 034412.98+320135.4$ & $378(29)$ & $1.8(0.4)$ & $0.37(0.05)$ & $0.12(0.02)$ & & \\
\hline Per-emb 63 & J032843.28+311733.0 & $436(9)$ & $1.9(0.4)$ & $0.36(0.05)$ & $0.25(0.02)$ & Bolo $31^{\mathrm{a}}$ & \\
\hline
\end{tabular}


Table 2

(Continued)

\begin{tabular}{|c|c|c|c|c|c|c|c|}
\hline ID & $\begin{array}{c}\text { c2d Name/Position } \\
(\text { SSTc2dJ...) }\end{array}$ & $\begin{array}{l}T_{\text {bol }} \\
(\mathrm{K})\end{array}$ & $\begin{array}{l}L_{\text {bol }} \\
\left(L_{\odot}\right)\end{array}$ & $\alpha_{\mathrm{IR}}$ & $\begin{array}{l}M_{\text {env }} \\
\left(M_{\odot}\right)\end{array}$ & Bolocam ID & Other Names \\
\hline Per-emb 64 & $\mathrm{~J} 033312.85+312124.1$ & $438(8)$ & $3.2(0.6)$ & $0.41(0.05)$ & $0.53(0.07)$ & Bolo 76 & \\
\hline Per-emb 65 & J032856.31+312227.8 & $440(191)$ & $0.16(0.16)$ & $-0.14(0.05)$ & $0.25(0.02)$ & & \\
\hline Per-emb 66 & J034345.15+320358.6 & $542(110)$ & $0.69(0.22)$ & $-0.22(0.05)$ & $0.24(0.02)$ & & \\
\hline
\end{tabular}

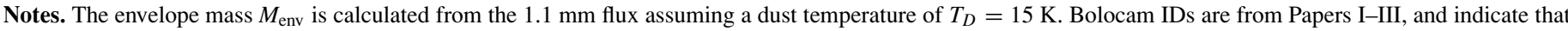

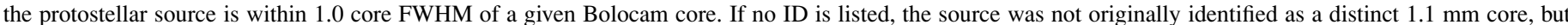

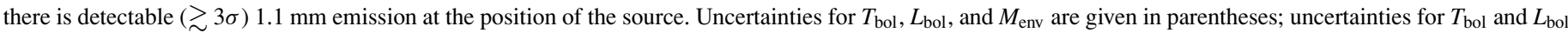

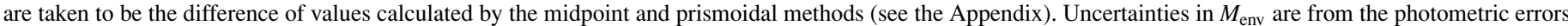

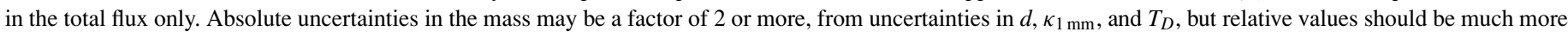
accurate.

a More than one protostar is associated with this Bolocam core or source is in a crowded region; mass is based on flux measured in a 30 " -40 " diameter aperture.

b More than one protostar is associated with this Bolocam core; mass is based on dividing Bolocam core flux equally (source separation is less than $30^{\prime \prime}$ ).

${ }^{c}$ Saturated $24 \mu \mathrm{m}$ flux has been replaced with IRAS $25 \mu \mathrm{m}$ flux for the calculation of $L_{\mathrm{bol}}$ and $T_{\mathrm{bol}}$.

${ }^{\mathrm{d}}$ Saturated at either $24 \mu \mathrm{m}$ or $70 \mu \mathrm{m}$ so $L_{\text {bol }}$ is likely an underestimate, but the IRAS beam is too confused to measure reliable $25 \mu \mathrm{m}$ or $60 \mu \mathrm{m}$ fluxes.

Table 3

Bolometric Temperatures, Luminosities, and Envelope Masses of Cold Protostars in Serpens

\begin{tabular}{|c|c|c|c|c|c|c|c|}
\hline ID & $\begin{array}{l}\text { c2d Name/Position } \\
\text { (SSTc2dJ...) }\end{array}$ & $\begin{array}{l}T_{\text {bol }} \\
(\mathrm{K}) \\
\end{array}$ & $\begin{array}{l}L_{\text {bol }} \\
\left(L_{\odot}\right)\end{array}$ & $\alpha_{\mathrm{IR}}$ & $\begin{array}{l}M_{\text {env }} \\
\left(M_{\odot}\right)\end{array}$ & Bolocam ID & Other Names \\
\hline \multicolumn{8}{|c|}{ Class 0} \\
\hline Ser-emb 1 & $\mathrm{~J} 182909.24+003132.3$ & $39(2)$ & $1.6(0.1)$ & $2.27(0.08)$ & $1.16(0.02)$ & Bolo 15 & \\
\hline Ser-emb 2 & $\mathrm{~J} 182952.44+003611.7$ & $42(10)$ & $1.0(0.1)$ & $0.77(0.05)$ & $0.54(0.03)$ & Bolo $24^{\mathrm{a}}$ & \\
\hline Ser-emb 3 & $\mathrm{~J} 182854.84+002952.5$ & $51(12)$ & $2.6(0.1)$ & $1.91(0.06)$ & $1.54(0.15)$ & Bolo $8^{\mathrm{a}}$ & \\
\hline Ser-emb 4 & $\mathrm{~J} 183000.72+011301.4$ & $54(16)$ & $1.2(1.6)$ & $1.68(0.08)$ & $2.56(0.03)$ & Bolo $28^{a}$ & \\
\hline Ser-emb 5 & $\mathrm{~J} 182854.84+001832.6$ & $56(12)$ & $0.16(0.1)$ & $0.9(0.06)$ & $0.24(0.02)$ & Bolo $7^{\mathrm{a}}$ & \\
\hline Ser-emb 6 & J182949.56+011521.9 & $56(12)$ & $11.0(6.0)$ & $2.65(0.07)$ & $7.98(0.07)$ & Bolo $23^{a}$ & FIRS1 \\
\hline Ser-emb 7 & $\mathrm{~J} 182854.12+002929.9$ & $58(13)$ & $3.1(0.1)$ & $1.36(0.06)$ & $1.67(0.17)$ & Bolo $8^{\mathrm{a}}$ & \\
\hline Ser-emb $8^{b}$ & $\mathrm{~J} 182948.12+011644.9$ & $58(16)$ & $2.1(2.4)$ & $1.37(0.06)$ & $3.72(0.37)$ & Bolo $22^{\mathrm{a}}$ & S68N \\
\hline Ser-emb 9 & $\mathrm{~J} 182855.92+002944.7$ & $66(21)$ & $0.34(0.63)$ & $1.89(0.06)$ & $1.19(0.12)$ & Bolo $8^{\mathrm{a}}$ & \\
\hline \multicolumn{8}{|c|}{ Class I } \\
\hline Ser-emb $10^{\mathrm{c}, \mathrm{d}}$ & $\mathrm{J} 182845.12+005203.5$ & $75(14)$ & $1.09(0.05)$ & $1.33(0.06)$ & $0.29(0.03)$ & Bolo $3^{\mathrm{a}}$ & IRAS $1862+0050$ \\
\hline Ser-emb 11 & $\mathrm{~J} 182906.72+003034.3$ & $77(12)$ & $1.9(1.2)$ & $1.66(0.06)$ & $1.39(0.14)$ & Bolo $14^{\mathrm{a}}$ & \\
\hline Ser-emb $12^{\mathrm{b}}$ & $\mathrm{J} 182952.08+011547.8$ & $85(9)$ & $2.6(1.2)$ & $1.54(0.06)$ & $1.23(0.12)$ & Bolo $23^{\mathrm{a}}$ & NSMM 10 IR \\
\hline Ser-emb 13 & $\mathrm{~J} 182902.04+003120.6$ & $86(31)$ & $0.05(0.09)$ & $0.24(0.06)$ & $0.17(0.02)$ & & \\
\hline Ser-emb 14 & $\mathrm{~J} 183005.40+004104.5$ & $100(32)$ & $0.08(0.1)$ & $1.3(0.07)$ & $0.19(0.02)$ & & \\
\hline Ser-emb 15 & $\mathrm{~J} 182954.24+003601.3$ & $101(43)$ & $0.17(0.27)$ & $-0.18(0.06)$ & $0.48(0.05)$ & Bolo $24^{\mathrm{a}}$ & \\
\hline Ser-emb $16^{\mathrm{d}}$ & $\mathrm{J} 182844.76+005125.7$ & $110(26)$ & $0.04(0.6)$ & $1.07(0.05)$ & $0.24(0.02)$ & Bolo $3^{\mathrm{a}}$ & \\
\hline Ser-emb 17 & $\mathrm{~J} 182906.36+003043.2$ & $117(21)$ & $1.5(1.3)$ & $1.7(0.06)$ & $1.38(0.14)$ & Bolo $14^{\mathrm{a}}$ & \\
\hline Ser-emb 18 & $\mathrm{~J} 182952.80+011456.0$ & $120(15)$ & $1.2(0.8)$ & $1.49(0.05)$ & $0.74(0.07)$ & Bolo $23^{\mathrm{a}}$ & \\
\hline Ser-emb 19 & $\mathrm{~J} 183000.00+011311.6$ & $129(29)$ & $2.0(1.9)$ & $2.57(0.06)$ & $2.56(0.03)$ & Bolo $28^{\mathrm{a}}$ & \\
\hline Ser-emb $20^{\mathrm{b}}$ & $\mathrm{J} 182949.20+011619.8$ & $131(3)$ & $4.4(2.1)$ & $3.8(0.11)$ & $1.53(0.15)$ & Bolo $22^{\mathrm{a}}$ & \\
\hline Ser-emb 21 & $\mathrm{~J} 182951.00+011640.6$ & $141(37)$ & $1.7(1.5)$ & $1.04(0.05)$ & $1.84(0.18)$ & Bolo $22^{\mathrm{a}}$ & \\
\hline Ser-emb 22 & $\mathrm{~J} 182957.48+011300.5$ & $157(39)$ & $2.6(2.2)$ & $1.01(0.05)$ & $2.35(0.24)$ & Bolo $25^{a}$ & \\
\hline Ser-emb 23 & $\mathrm{~J} 182957.84+011251.4$ & $190(23)$ & $3.1(1.5)$ & $0.67(0.05)$ & $1.06(0.11)$ & Bolo $25^{\mathrm{a}}$ & \\
\hline Ser-emb 24 & $\mathrm{~J} 183000.00+011158.9$ & $249(64)$ & $1.8(1.2)$ & $1.15(0.05)$ & $1.14(0.11)$ & Bolo 29 & \\
\hline Ser-emb 25 & $\mathrm{~J} 182851.24+001927.3$ & $250(106)$ & $0.06(0.07)$ & $0.45(0.05)$ & $0.16(0.02)$ & Bolo $7^{\mathrm{a}}$ & \\
\hline Ser-emb 26 & $\mathrm{~J} 182958.92+011426.2$ & 303 (139) & $0.62(0.73)$ & $0.46(0.05)$ & $2.0(0.2)$ & Bolo $26^{\mathrm{a}}$ & \\
\hline Ser-emb $27^{b}$ & $\mathrm{~J} 182956.76+011446.5$ & $322(25)$ & $5.8(1.7)$ & $0.3(0.05)$ & $0.69(0.07)$ & & \\
\hline Ser-emb $28^{d}$ & $\mathrm{~J} 182844.04+005337.9$ & $360(244)$ & $0.19(1.5)$ & $0.45(0.05)$ & $0.26(0.03)$ & Bolo 2 & \\
\hline Ser-emb 29 & $\mathrm{~J} 183002.88+011228.2$ & $374(101)$ & $2.2(1.3)$ & $0.21(0.05)$ & $1.32(0.13)$ & Bolo $28^{\mathrm{a}}$ & \\
\hline Ser-emb 30 & $\mathrm{~J} 182957.84+011405.7$ & $560(85)$ & $13.8(4.2)$ & $0.28(0.09)$ & $1.9(0.19)$ & Bolo $26^{\mathrm{a}}$ & IRAS $18274+0112$ \\
\hline Ser-emb 31 & $\mathrm{~J} 182931.92+011842.9$ & $430(15)$ & $5.3(1.0)$ & $0.26(0.05)$ & $2.12(0.07)$ & Bolo 20 & \\
\hline Ser-emb 32 & $\mathrm{~J} 183005.76+003931.7$ & 431 (207) & $0.05(0.07)$ & $-0.39(0.05)$ & $0.2(0.02)$ & Bolo 32 & \\
\hline Ser-emb $33^{\mathrm{e}}$ & $\mathrm{J} 182916.08+001822.7$ & $437(10)$ & $1.7(0.2)$ & $-0.07(0.05)$ & $0.54(0.04)$ & Bolo 17 & IRAS $18267+0016$ \\
\hline Ser-emb 34 & $\mathrm{~J} 182902.76+003009.5$ & $631(410)$ & $0.12(0.28)$ & $-0.14(0.05)$ & $0.22(0.02)$ & Bolo 13 & \\
\hline
\end{tabular}

Notes. Columns are as in Table 2, and uncertainties for $T_{\mathrm{bol}}, L_{\mathrm{bol}}$, and $M_{\mathrm{env}}$ are given in parentheses.

${ }^{a}$ More than one protostar is associated with this Bolocam core or source is in a crowded region; mass is based on flux measured in a $30^{\prime \prime}-40^{\prime \prime}$ diameter aperture.

${ }^{\mathrm{b}}$ Saturated at either $24 \mu \mathrm{m}$ or $70 \mu \mathrm{m}$ so $L_{\mathrm{bol}}$ is likely an underestimate, but the IRAS beam is too confused to measure reliable $25 \mu \mathrm{m}$ or $60 \mu \mathrm{m}$ fluxes.

${ }^{\mathrm{c}}$ Missing $70 \mu \mathrm{m}$ flux has been replaced with IRAS $60 \mu \mathrm{m}$ flux for the calculation of $L_{\mathrm{bol}}$ and $T_{\mathrm{bol}}$.

${ }^{\mathrm{d}}$ Source lies outside the $70 \mu \mathrm{m}$ map.

${ }^{\mathrm{e}}$ Saturated $24 \mu \mathrm{m}$ flux has been replaced with IRAS $25 \mu \mathrm{m}$ flux for the calculation of $L_{\mathrm{bol}}$ and $T_{\mathrm{bol}}$. 
Table 4

Bolometric Temperatures, Luminosities, and Envelope Masses of Cold Protostars in Ophiuchus

\begin{tabular}{|c|c|c|c|c|c|c|c|}
\hline ID & $\begin{array}{c}\text { c2d Name/Position } \\
(\text { SSTc2dJ...) }\end{array}$ & $\begin{array}{l}T_{\text {bol }} \\
(\mathrm{K}) \\
\end{array}$ & $\begin{array}{l}L_{\text {bol }} \\
\left(L_{\odot}\right)\end{array}$ & $\alpha_{\mathrm{IR}}$ & $\begin{array}{l}M_{\text {env }} \\
\left(M_{\odot}\right) \\
\end{array}$ & Bolocam ID & Other Names \\
\hline \multicolumn{8}{|c|}{ Class 0} \\
\hline Oph-emb 1 & $\mathrm{~J} 162821.72-243623.4$ & $35(2)$ & $0.25(0.01)$ & $1.23(0.06)$ & $0.51(0.04)$ & Bolo 26 & IRAS $16253-2429$ \\
\hline Oph-emb $2^{\mathrm{a}}$ & J163222.56-242831.8 & $54(8)$ & $6.9(1.2)$ & $5.03(0.2)$ & $1.16(0.02)$ & Bolo 36 & IRAS $16293-2422 B$ \\
\hline Oph-emb 3 & J162625.80-242428.8 & $57(10)$ & $0.41(0.15)$ & $1.65(0.07)$ & $0.24(0.02)$ & Bolo 9 & VLA $1623(?)$ \\
\hline \multicolumn{8}{|c|}{ Class I } \\
\hline Oph-emb 4 & $\mathrm{~J} 163136.84-240419.9$ & $77(25)$ & $0.18(0.05)$ & $-0.27(0.05)$ & $0.03(0.01)$ & & \\
\hline Oph-emb 5 & J162721.96-242727.7 & $87(28)$ & $0.08(0.08)$ & $-0.05(0.05)$ & $0.12(0.01)$ & Bolo 17 & \\
\hline Oph-emb 6 & J162705.40-243629.5 & $106(14)$ & $0.15(0.09)$ & $1.27(0.05)$ & $0.09(0.01)$ & Bolo $14^{\mathrm{b}}$ & LFAM26/GY197 \\
\hline Oph-emb 7 & $\mathrm{~J} 163151.96-245725.9$ & $124(39)$ & $0.1(0.13)$ & $0.82(0.05)$ & $0.05(0.01)$ & Bolo 33 & \\
\hline Oph-emb $8^{\mathrm{c}, \mathrm{d}}$ & $\mathrm{J} 162621.48-242304.2$ & $133(34)$ & $18.3(1.4)$ & $1.46(0.09)$ & $0.22(0.02)$ & Bolo 5 & GSS 30 \\
\hline Oph-emb 9 & J162625.44-242301.3 & $135(49)$ & $0.1(0.3)$ & $0.87(0.05)$ & $0.53(0.05)$ & Bolo 8 & GY 30 \\
\hline Oph-emb 10 & $\mathrm{~J} 163200.96-245642.7$ & $145(38)$ & $2.7(0.3)$ & $1.39(0.07)$ & $0.05(0.01)$ & Bolo 35 & L1689S/IRS67 \\
\hline Oph-emb 11 & J162717.64-242856.2 & $190(1)$ & $0.52(0.06)$ & $0.25(0.05)$ & $0.07(0.01)$ & & \\
\hline Oph-emb 12 & $\mathrm{~J} 162724.48-244103.1$ & $191(30)$ & $0.33(0.16)$ & $1.01(0.05)$ & $0.12(0.01)$ & Bolo $18^{b}$ & CRBR85 \\
\hline Oph-emb $13^{c}$ & $\mathrm{~J} 162728.08-243933.4$ & $193(31)$ & $7.2(0.5)$ & $2.29(0.11)$ & $0.11(0.01)$ & & IRS 44/GY269 \\
\hline Oph-emb $14^{c}$ & $\mathrm{~J} 162727.00-244050.5$ & $211(7)$ & $3.8(0.7)$ & $1.17(0.08)$ & $0.36(0.04)$ & Bolo $18^{b}$ & IRS 43/GY265 \\
\hline Oph-emb 15 & J163152.32-245536.1 & $240(42)$ & $0.13(0.07)$ & $1.07(0.05)$ & $0.08(0.01)$ & & \\
\hline Oph-emb $16^{\mathrm{c}, \mathrm{d}}$ & $\mathrm{J} 162709.36-243718.4$ & $257(60)$ & $17.9(0.6)$ & $1.69(0.15)$ & $0.07(0.01)$ & Bolo $14^{\mathrm{b}}$ & EL 29/GY214 \\
\hline Oph-emb 17 & J163135.76-240129.2 & $290(18)$ & $1.5(0.1)$ & $0.14(0.05)$ & $0.14(0.02)$ & Bolo 30 & IRAS $16295-2355$ \\
\hline Oph-emb 18 & J162857.72-244054.8 & $304(96)$ & $0.03(0.03)$ & $0.67(0.05)$ & $0.04(0.01)$ & & \\
\hline Oph-emb 19 & $\mathrm{~J} 162728.44-242720.8$ & $309(85)$ & $0.47(0.28)$ & $-0.03(0.05)$ & $0.29(0.03)$ & Bolo $20^{\mathrm{b}}$ & IRS45/GY273 \\
\hline Oph-emb 20 & $\mathrm{~J} 162706.84-243815.0$ & $310(23)$ & $0.6(0.16)$ & $0.61(0.05)$ & $0.05(0.01)$ & Bolo 13 & WL17/GY205 \\
\hline Oph-emb $21^{\mathrm{c}}$ & J162702.16-243727.1 & $331(2)$ & $5.2(1.3)$ & $1.53(0.11)$ & $0.08(0.01)$ & & WL16/GY182 \\
\hline Oph-emb 22 & J162640.56-242714.4 & $372(160)$ & $0.06(0.09)$ & $0.45(0.05)$ & $0.06(0.01)$ & & GY91/CRBR42 \\
\hline Oph-emb 23 & J162648.48-242838.6 & $428(84)$ & $0.12(0.05)$ & $0.02(0.05)$ & $0.03(0.01)$ & & WL2/GY128 \\
\hline Oph-emb 24 & J162737.08-244237.8 & $438(87)$ & $0.13(0.06)$ & $0.13(0.05)$ & $0.04(0.01)$ & & GY301 \\
\hline Oph-emb 25 & J163143.68-245524.6 & $496(68)$ & $0.28(0.09)$ & $0.23(0.05)$ & $0.04(0.01)$ & & \\
\hline Oph-emb 26 & $\mathrm{~J} 162730.24-242743.2$ & $528(99)$ & $0.93(0.27)$ & $-0.12(0.05)$ & $0.19(0.02)$ & Bolo $20^{\mathrm{b}}$ & IRS47/GY279 \\
\hline Oph-emb 27 & J162739.96-244314.8 & $551(54)$ & $0.74(0.15)$ & $-0.15(0.05)$ & $0.04(0.01)$ & & IRS51/GY315 \\
\hline Oph-emb 28 & J162721.60-244143.0 & $595(57)$ & $1.2(0.2)$ & $-0.03(0.05)$ & $0.04(0.01)$ & & IRS42/GY252 \\
\hline
\end{tabular}

Notes. Columns are as in Table 2, and uncertainties for $T_{\mathrm{bol}}, L_{\mathrm{bol}}$, and $M_{\mathrm{env}}$ are given in parentheses.

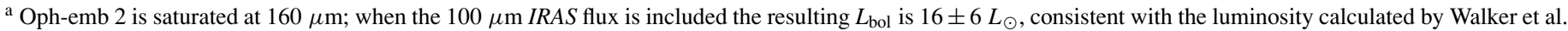
(1986) when scaled to a distance of $125 \mathrm{pc}$.

b More than one protostar is associated with this Bolocam core or source is in a crowded region; mass is based on flux measured in a $30^{\prime \prime}-40^{\prime \prime}$ diameter aperture.

c Saturated $24 \mu \mathrm{m}$ flux has been replaced with IRAS $25 \mu \mathrm{m}$ flux for the calculation of $L_{\mathrm{bol}}$ and $T_{\mathrm{bol}}$.

${ }^{\mathrm{d}}$ Saturated $70 \mu \mathrm{m}$ flux has been replaced with IRAS $60 \mu \mathrm{m}$ flux for the calculation of $L_{\mathrm{bol}}$ and $T_{\text {bol }}$.

an accurate determination of $L_{\text {submm }}$ is not feasible with these data. ${ }^{1}$ We will focus, therefore, on $T_{\mathrm{bol}}$ and $\alpha_{\mathrm{IR}}$.

\subsection{Comparing Classification Methods}

In Figure 4, we compare classifications based on $\alpha_{\mathrm{IR}}$ and $T_{\text {bol }}$ for the candidate embedded protostars in Perseus, Serpens, and Ophiuchus. Thick symbols indicate sources associated with $1.1 \mathrm{~mm}$ emission, while thin symbols denote sources with upper limits at $1.1 \mathrm{~mm}$. The spectral index $\alpha_{\mathrm{IR}}$ is determined from a least-squares fit to all detections between $\lambda=2 \mu \mathrm{m}$ and $\lambda=24 \mu \mathrm{m}$. The calculation of $T_{\mathrm{bol}}$ is described in Section 3.1 and Appendix. We adopt $T_{\text {bol }}$ class divisions from Chen et al. (1995) (see Table 1), and $\alpha_{\mathrm{IR}}$ divisions from André \& Montmerle (1994): $\alpha_{\mathrm{IR}}<0$ (Class I) and $\alpha_{\mathrm{IR}}>0$ (Class II). No well-defined $\alpha_{\mathrm{IR}}$ criteria exist for Class 0 sources, as deeply embedded objects were generally not visible in the mid-IR prior to Spitzer.

The two classification methods agree fairly well for Class II and "warmer" Class I sources. Sources with $T_{\text {bol }} \lesssim 100 \mathrm{~K}$, by contrast, have a wide range of $\alpha_{\mathrm{IR}}$ values, and a few of these "cold" sources even fall into Class II based on the spectral index.

\footnotetext{
5 It is possible to calculate $L_{\text {submm }}$ by assuming a modified blackbody spectrum and a value for $\beta$ (e.g., Hatchell et al. 2007b), but given the assumptions involved we choose not to pursue this method.
}

One of the reasons for this large scatter in $\alpha_{\mathrm{IR}}$ becomes apparent when examining the SEDs of some deeply embedded sources, which show considerable differences at short wavelengths. Many protostellar SEDs are not monotonically increasing from 3.6 to $24 \mu \mathrm{m}$, often falling from 5.8 to $8 \mu \mathrm{m}$ and rising again at longer wavelengths (e.g., Per-emb 18, Per-emb 21, Oph-emb 1; Figure 1). Geometric effects such as scattered light from an outflow cavity or absorption from ices in the protostellar envelope are the most likely cause of these features. In any case, nonmonotonic behavior at short wavelengths will clearly bias the calculation of $\alpha_{\mathrm{IR}}$. Calculating $\alpha_{\mathrm{IR}}$ from a straight line fit between 2 and $24 \mu \mathrm{m}$, rather than a least-squares fit, still results in a large range of $\alpha_{\mathrm{IR}}$ values at low $T_{\mathrm{bol}}$.

To determine whether $\alpha_{\mathrm{IR}}$ or $T_{\mathrm{bol}}$ is a more accurate measure of the true evolutionary state, we look at the correlation between both measures and the degree to which a given source is embedded. Figure 5 shows the distribution of $T_{\text {bol }}$ with respect to the distance from each protostellar candidate to the nearest $1.1 \mathrm{~mm}$ core, in units of the core FWHM size. In all three clouds, essentially all sources with $T_{\text {bol }} \lesssim 200 \mathrm{~K}$ are located within one core FWHM of a $1.1 \mathrm{~mm}$ peak. Thus the majority of "cold" objects, as defined by low $T_{\text {bol }}$, appear to be embedded in dense envelopes, and are likely to be at an early evolutionary stage. Furthermore, there is a clear correlation between smaller 


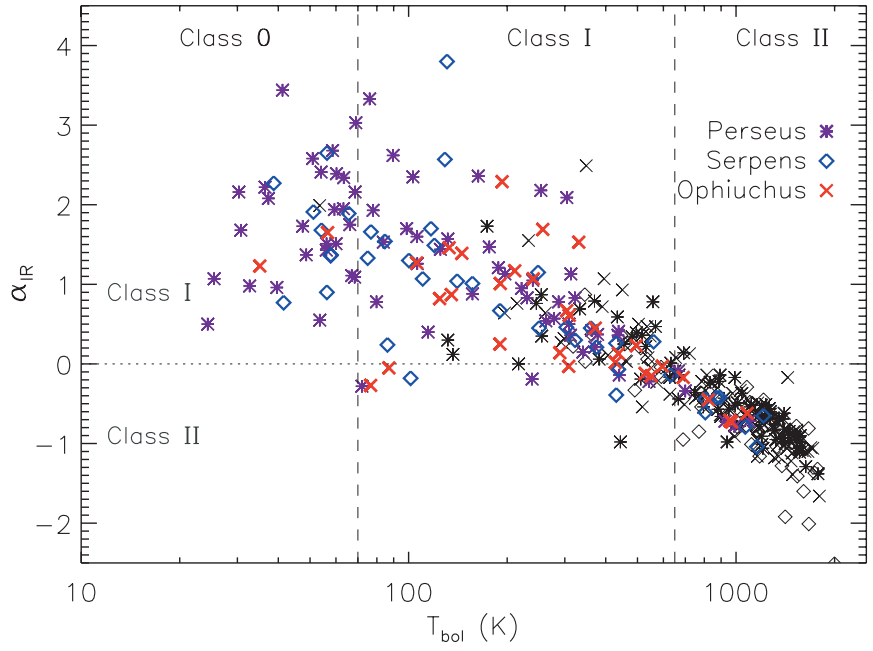

Figure 4. Near- to mid-IR spectral index $\left(\alpha_{\mathrm{IR}}\right)$ vs. bolometric temperature $\left(T_{\text {bol }}\right)$ for the candidate embedded protostar samples in Perseus, Serpens, and Ophiuchus. The bold symbols indicate sources that are associated with $1.1 \mathrm{~mm}$ emission, and the thin symbols denote those with upper limits at $1.1 \mathrm{~mm}$. Standard class divisions for both $T_{\text {bol }}$ and $\alpha_{\mathrm{IR}}$ are shown. The two methods agree fairly well for Class II and "warmer" Class I sources, but very cold ( $T_{\text {bol }} \lesssim 100 \mathrm{~K}$ ) sources have a large range of $\alpha_{\mathrm{IR}}$ values.

(A color version of this figure is available in the online journal.)

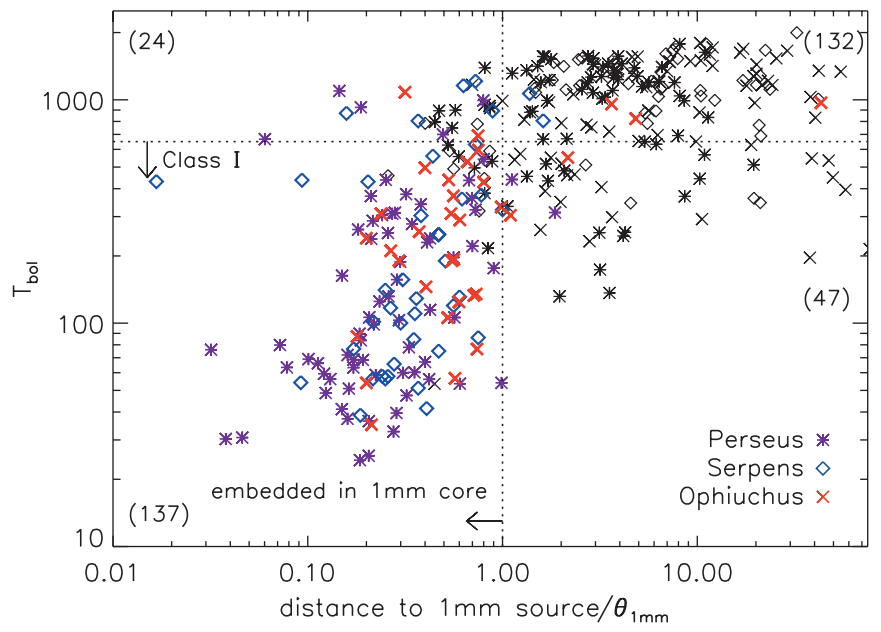

Figure 5. Distribution of $T_{\mathrm{bol}}$ as a function of the distance to the nearest $1.1 \mathrm{~mm}$ core, for candidate embedded protostars in all three clouds. Distances are in units of the $1.1 \mathrm{~mm}$ core FWHM size $\left(\theta_{1 \mathrm{~mm}}\right)$, and symbols are as in Figure 4. Sources within $1.0 \times \theta_{1 \mathrm{~mm}}$ of a $1.1 \mathrm{~mm}$ core position are considered embedded within that core. There is a clear correlation between smaller distances, or more embedded sources, and lower $T_{\text {bol }}$ values, suggesting that $T_{\text {bol }}$ is a good measure of evolutionary state for deeply embedded sources. For reference, the total numbers of sources in each quadrant of the plot are given in parentheses.

(A color version of this figure is available in the online journal.)

distance and lower $T_{\mathrm{bol}}$, suggesting that sources with lower $T_{\mathrm{bol}}$ are more embedded than those with higher $T_{\text {bol }}$. This correlation remains even if we exclude the $1.1 \mathrm{~mm}$ flux from the calculation of $T_{\text {bol }}$, eliminating the possible bias between millimeter cores and $T_{\text {bol }}$. Thus, if we affiliate the degree of embeddedness with youth, then $T_{\text {bol }}$ appears to correlate well with evolutionary state.

Figure 6 is similar, for $\alpha_{\mathrm{IR}}$; while there is some correlation between higher $\alpha_{\mathrm{IR}}$ values and smaller distances to the nearest $1.1 \mathrm{~mm}$ core, the relationship is not nearly as clear as for $T_{\text {bol }}$. In particular, there are a number of sources with large $\alpha_{\mathrm{IR}}$ values that do not seem to be embedded (the distance to

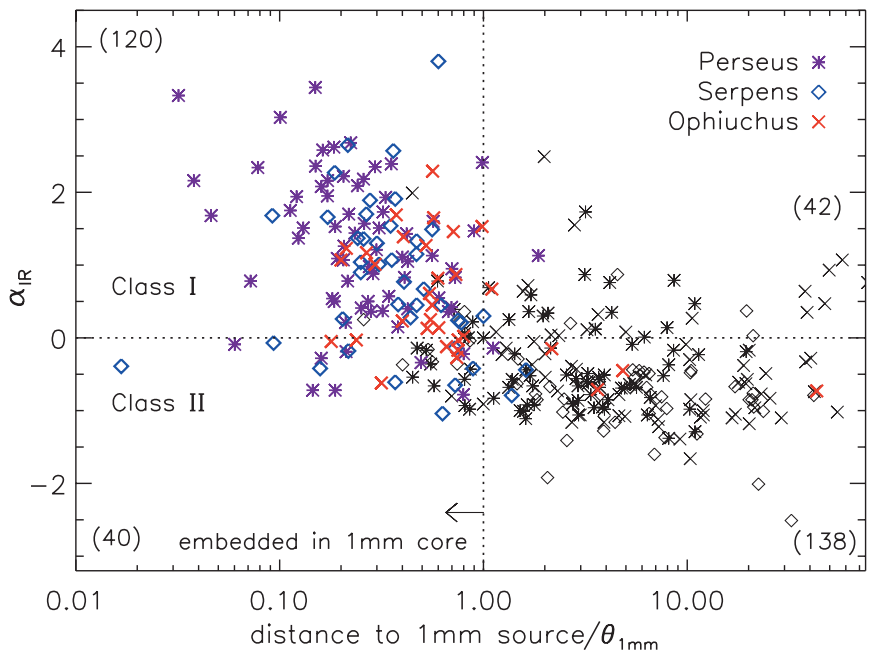

Figure 6. Distribution of $\alpha_{\mathrm{IR}}$ as a function of the distance to the nearest $1.1 \mathrm{~mm}$ core for embedded protostar candidates in all three clouds, similar to Figure 5. While there is some correlation between higher $\alpha_{\mathrm{IR}}$ values and smaller distances, it is not as compelling as the correlation observed for $T_{\text {bol }}$ (Figure 5). For reference, the total numbers of sources in each quadrant are given in parentheses. (A color version of this figure is available in the online journal.)

the nearest core is much larger than one FWHM), and there are several deeply embedded sources with low $\alpha_{\mathrm{IR}}$ values. Based on Figures 4-6 and a visual examination of sources with $T_{\text {bol }}<70 \mathrm{~K}$, a number of which are known Class 0 sources, we conclude that $T_{\text {bol }}$ is more reliable than $\alpha_{\mathrm{IR}}$ for classifying deeply embedded protostars. Hereafter, $T_{\text {bol }}$ will be used to characterize protostellar sources, and we use the divisions from Chen et al. (1995) and Table 1 to place sources into Class 0, Class I, or Class II.

\section{CHARACTERISTICS OF CLASS 0, I, AND II SOURCES}

The total numbers of Class 0 and Class I sources in each cloud, as defined by $T_{\text {bol }}$ classifications, are given in Table 6 . Note that in addition to $T_{\text {bol }}$, we also require Class I sources to be detected at $1.1 \mathrm{~mm}$. This is not an unreasonable requirement, as our definition of Stage I dictates that they should be detected at millimeter wavelengths $\left(M_{\text {env }}>0.1 M_{\odot}\right)$. The individual spectra (for $\lambda=1.25-1100 \mu \mathrm{m}$ ) of all Class 0 and Class I sources are shown in Figure 7.

Numbers in parentheses in Table 6 are based on classifications using the prismoidal, rather than midpoint, method for calculating $T_{\text {bol }}$; these give some idea of the uncertainties in the number of Class 0 and Class I sources. Despite the sometimes substantial difference in the midpoint and prismoidal $T_{\text {bol }}$ values for individual sources, there is very little difference in the resulting number of Class 0 and Class I protostars, with the exception of Serpens where the number of Class 0 sources increases from 10 to 14 .

Statistics for Class II are not given; as we intended to select against sources without a protostellar envelope, we are necessarily incomplete to these objects. For example, there are 46 sources in our original Serpens candidate sample with $T_{\text {bol }}>650 \mathrm{~K}$, while Harvey et al. (2007a) find 132 Class II sources. We do detect a few Class II sources at $1.1 \mathrm{~mm}$, however (see Section 5.1).

The average spectra of Class 0, Class I, and Class II sources in Perseus and Serpens are shown in Figure 8. To calculate the average spectra, individual SEDs are weighted by $1 / L_{\text {bol }}$, so that we are not biased by the most luminous sources. Error bars in 

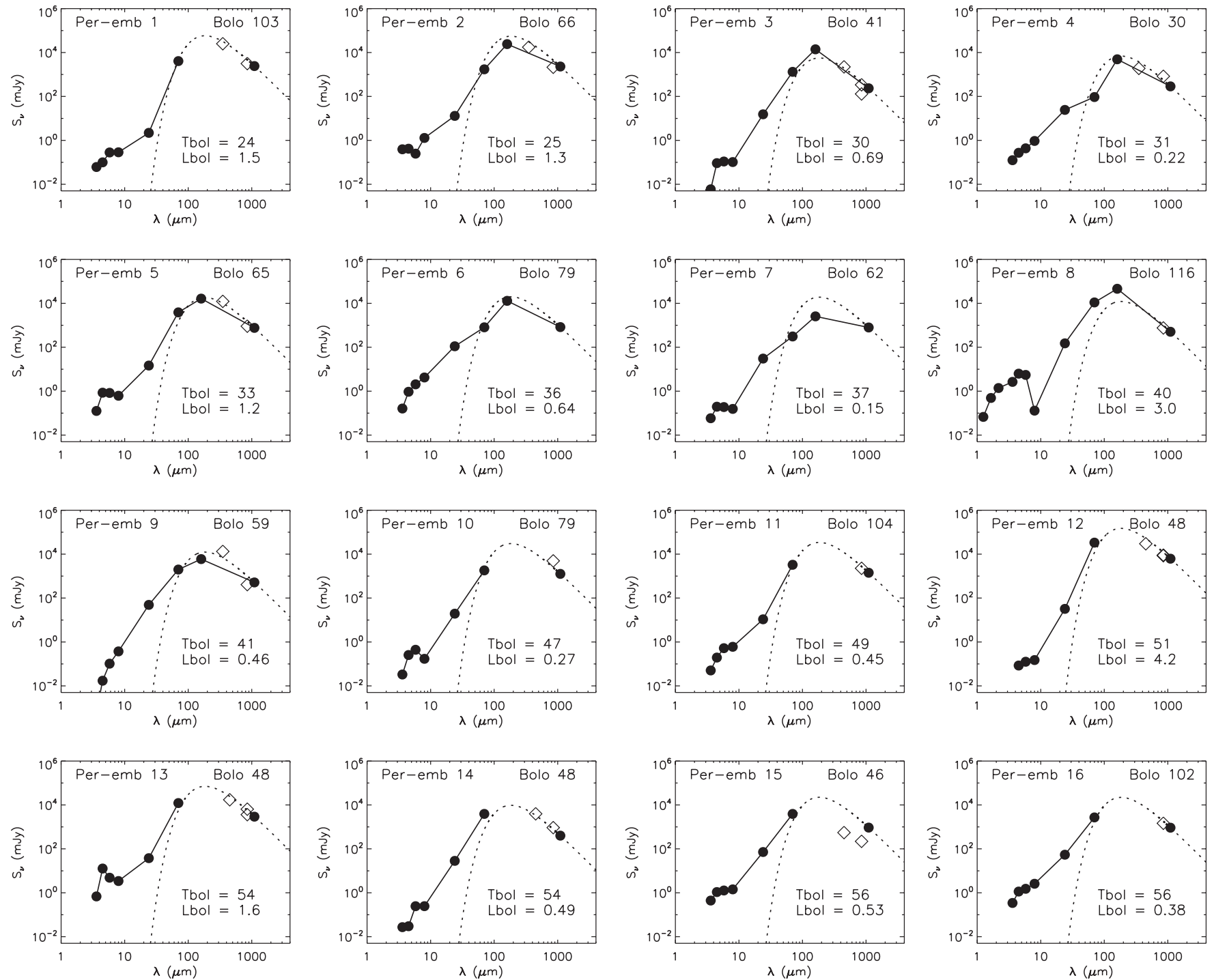

Figure 7. SEDs of all embedded protostars in Perseus, Serpens, and Ophiuchus, including photometry from 2MASS (1.25, 1.65, 2.17 $\mu \mathrm{m})$, IRAC (3.6, 4.5, 5.8, $8.0 \mu \mathrm{m})$, MIPS $(24,70,160 \mu \mathrm{m})$, and Bolocam $(1.1 \mathrm{~mm})$ data. Also included are SCUBA 450 and $850 \mu \mathrm{m}$ fluxes from the literature (Sandell \& Knee 2001 ; Kirk et al. 2006) and IRAS 25 or $60 \mu \mathrm{m}$ fluxes where they are used to replace saturated 24 or $70 \mu \mathrm{m}$ fluxes (see Section 3.1). A sample is shown here.

(An extended version of this figure is available in the online journal.)

Figure 8 represent the $1 \sigma$ error in the mean $\left(\sigma_{\lambda} / \sqrt{N_{\lambda}}\right)$, but the dispersion in the sample $\left(\sigma_{\lambda}\right)$ is much larger. Source SED shapes do not fall into discrete bins, but form a continuous distribution between the averages shown. Error bars are large at wavelengths where many sources are not detected (at short wavelengths for Class 0, long wavelengths for Class II, and $160 \mu \mathrm{m}$ for all classes), and where there are significant variations from source to source.

If Ophiuchus sources are included in the average calculation, the resulting average spectra are skewed toward having more flux at short wavelengths, have larger dispersion, and the similarity of SEDs within each bin is reduced. This behavior suggests that $T_{\text {bol }}$ may be biased for many sources in Ophiuchus, likely due to the lack of information at $160 \mu \mathrm{m}$, so we exclude Ophiuchus from the average spectra.

The progression from Class 0 to Class I to Class II is consistent with a sequence of physical evolution. In particular, the average Class 0 spectrum has the lowest flux densities from 1.25 to $24 \mu \mathrm{m}$, as expected for deeply embedded sources with massive, extincting envelopes, and the highest fluxes at $70-1100 \mu \mathrm{m}$, where reprocessed protostellar flux is emitted by the cold envelope. By contrast, the average Class II spectrum is relatively flat, with a much larger percentage of the protostar flux emerging at shorter wavelengths, as expected for older sources without much circumstellar material. One must keep in mind, however, that $T_{\text {bol }}$ is defined such that spectra which peak at longer wavelengths will have a lower $T_{\mathrm{bol}}$.

Large error bars on the average Class 0 spectrum for $\lambda=$ $1-3 \mu \mathrm{m}$ are indicative of the widely varying behavior of Class 0 objects in the near-IR, and the presence of nonmonotonic behavior is apparent at $\lambda=3.6 \mu \mathrm{m}$. The large separation between the Class 0 and Class I spectra, as well as the continuous range of SED shapes, suggests that a more continuous means of estimating evolutionary status is preferable to the standard classes.

\subsection{Class II Sources with $1 \mathrm{~mm}$ Emission}

The average Class II spectrum is not necessarily representative of all Class II objects, due to severe incompleteness to nonembedded sources. The Class II sources we do detect are likely relatively young Stage II objects, before a substantial fraction of disk mass is dispersed or accreted. 
Given our $1.1 \mathrm{~mm}$ sensitivity limit of approximately $0.1 M_{\odot}$, almost all sources detected at $1.1 \mathrm{~mm}$ will be dense cores or envelopes around relatively young protostars. In general, by the time a protostar has consumed or dispersed its massive envelope and enters Stage II (or the T Tauri phase, e.g., Adams et al. 1987), the remnant disk of gas and dust has too little mass to be detected by our millimeter surveys. Crapsi et al. (2008) define Stage II as sources with a circumstellar mass below $0.1 \mathrm{M}_{\odot}$. Typical measured masses of Class II disks are $0.01-0.1 M_{\odot}$ (e.g., Beckwith \& Sargent 1996), although values as large as $1 M_{\odot}$ have been measured (Beckwith et al. 1990).

In a few cases, we do detect $1.1 \mathrm{~mm}$ emission around sources with Class II-type SEDs. These objects have bolometric temperatures $T_{\text {bol }}>650 \mathrm{~K}$ and a flux density at $1.1 \mathrm{~mm}$ that is lower than the flux densities from 3.6 to $24 \mu \mathrm{m}$. With the exception of two "flat spectrum" objects $\left(-0.3<\alpha_{\mathrm{IR}}<0.3\right.$; Greene et al. 1994), the near- to mid-IR spectral indices, $\alpha_{\mathrm{IR}}$, of these objects are in the range -0.34 to -1.04 , confirming their Class II status. In some cases, the $1.1 \mathrm{~mm}$ emission is unresolved, consistent with a compact disk. Often, however, these Class II sources are in a region of confused millimeter emission, so their physical association with the $1.1 \mathrm{~mm}$ emission is not secure.

Table 5 lists all Class II sources $\left(650<T_{\text {bol }}<2800 \mathrm{~K}\right)$ in each cloud that are detected in our $1.1 \mathrm{~mm}$ surveys. Those in confused regions of $1.1 \mathrm{~mm}$ emission are indicated by an "**” in the "Bolocam ID" column. Sources with pointlike $1.1 \mathrm{~mm}$ emission centered on the Spitzer position (three in Perseus, one in Serpens, and three in Ophiuchus) may have massive disks.

\subsection{Individual Sources}

Here, we briefly discuss a few examples of newly identified or otherwise interesting embedded protostellar sources.

\subsubsection{IRAS $03292+3039$}

IRAS 03292+3039 (Per-emb 2) is a little-studied, Class 0 source associated with the $1.1 \mathrm{~mm}$ core Per-Bolo 66; it was discussed briefly in Paper I. An image of IRAS 03292+3039, together with the SED, is shown in Figure 9. Jørgensen et al. (2006) identified this as an outflow source, noting the large-scale outflow visible in the $4.5 \mu \mathrm{m}$ IRAC band.

Bright $1.1 \mathrm{~mm}$ emission centered on this object indicates a fairly massive protostellar envelope $\left(2.9 M_{\odot}\right)$, while the powerful outflow (Walawender et al. 2005; Jørgensen et al. 2006; Hatchell et al. 2007a) and low bolometric temperature $\left(T_{\text {bol }}=25 \mathrm{~K}\right)$ are evidence of an extremely young, energetic embedded protostar. The SED is similar to well-known Class 0 protostars in Perseus such as NGC 1333-IRAS 4, and nearby IRAS $03282+3035$. The small fan-shaped nebulosity visible at 3.6 and $4.5 \mu \mathrm{m}$ (Figure 9) is most easily explained by a cone-shaped cavity, carved out of the dense envelope by an energetic outflow. The orientation of the nebulosity corresponds well to the larger-scale outflow traced by IRAC emission (Jørgensen et al. 2006), which is indicated by thick gray lines in Figure 9. The one-sided nebulosity at $3.6 \mu \mathrm{m}$ is strikingly similar to the Whitney et al. (2003) model IRAC image of an early Stage 0 sources viewed at an inclination angle of $30^{\circ}$ (their Figure 12(a)).

\subsubsection{Per-Bolo 102}

Per-Bolo 102 is a bright $1.1 \mathrm{~mm}$ source that was identified in the Bolocam survey of Perseus (Enoch et al. 2006). It lies within the region of active star formation near IC 348, which

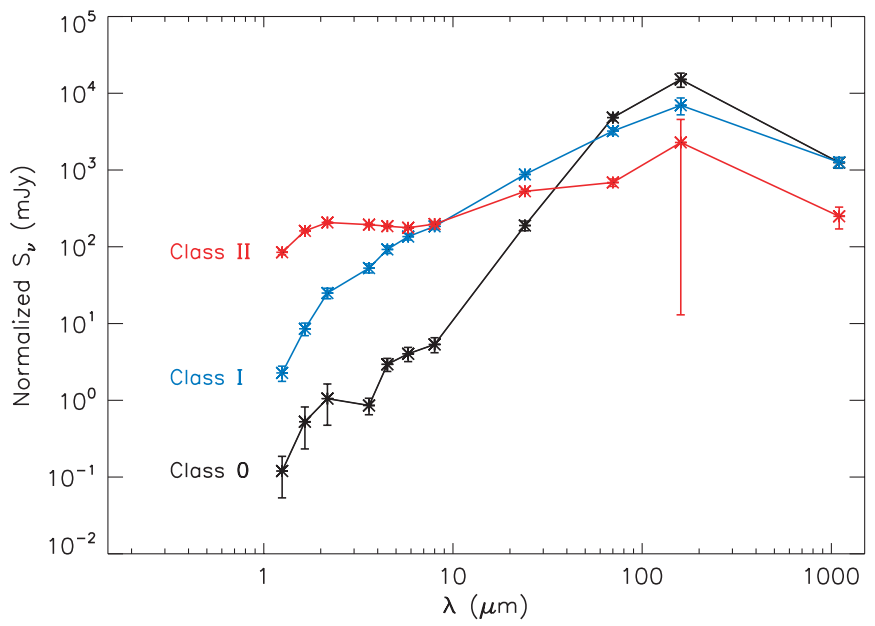

Figure 8. Average spectra of sources in Class 0, Class I, and Class II, for the Perseus and Serpens samples. Sources are classified based on bolometric temperature, with the additional requirement that Class I sources be detected at $1.1 \mathrm{~mm}$ (see Table 1). Individual SEDs are weighted by $1 / L_{\text {bol }}$ in the average calculation. Error bars are the $1 \sigma$ error in mean, and not the sample dispersion, which is much larger. The Class II spectrum is not necessarily representative of all Class II objects, as we are very incomplete to sources without protostellar envelopes.

(A color version of this figure is available in the online journal.)

includes the famous outflow-driving source $\mathrm{HH} 211$ (McCaughrean et al. 1994). The Spitzer $24 \mu \mathrm{m}$ image resolves the luminous internal source into a double object (Per-emb 18 and Per-emb 22). While the two SEDs are very similar for $\lambda \geqslant 8 \mu \mathrm{m}$ (Figure 9), variations at shorter wavelengths cause Per-emb $18\left(T_{\text {bol }}=\right.$ $56 \mathrm{~K})$ to fall into Class 0 , while Per-emb $22\left(T_{\text {bol }}=72 \mathrm{~K}\right)$ falls just outside the Class 0/Class I boundary. These sources are good examples of why a more continuous evolutionary scheme is preferable to the standard classifications.

Recently obtained CARMA interferometric observations at $\lambda=2.7 \mathrm{~mm}$ resolve the millimeter core into two sources, coincident with the Spitzer source positions (M. L. Enoch et al. 2009 , in preparation). The flux ratio at $2.7 \mathrm{~mm}$ of the northern (Per-emb 18) to southern (Per-emb 22) source is at least 2:1, further evidence that Per-emb 22 is slightly more evolved. Although not as massive or cold as nearby HH 211 (Per-emb 1) and IC 348-mms (Per-emb 11), Per-Bolo 102 is an interesting case study. It may be a separate-envelope binary system, with two nearly coeval Class 0 or early Class I sources. The sources are separated by $17^{\prime \prime}$, or 4200 AU. Binary separations of this order are consistent with early fragmentation in a relatively dense cloud ("prompt initial fragmentation," e.g., Pringle 1989; Looney et al. 2000), in which case the individual sources would have distinct protostellar envelopes.

In a binary formed via gravitational fragmentation, we would expect the separation to correspond to the local Jeans length (Jeans 1928):

$$
\lambda_{J}=\left(\frac{\pi c_{s}^{2}}{G \mu_{p} m_{H} n}\right)^{1 / 2},
$$

where $c_{s}$ is the local sound speed, and $\mu_{p}=2.33$ and $n$ are the mean molecular weight and mean particle density, respectively. A Jeans length of $4200 \mathrm{AU}$ would require a relatively high density $\left(n \sim 6 \times 10^{5} \mathrm{~cm}^{-3}\right.$, assuming $\left.c_{s}=0.2 \mathrm{~km} \mathrm{~s}^{-1}\right)$. The mean density of the Per-Bolo 102 core, measured within an aperture of $10^{4} \mathrm{AU}$, is $4 \times 10^{5} \mathrm{~cm}^{-3}$, close to the required value. 
Table 5

Class II Sources Detected at $1.1 \mathrm{~mm}$ in Perseus, Serpens, and Ophiuchus

\begin{tabular}{|c|c|c|c|c|c|c|}
\hline $\begin{array}{l}\text { c2d Name/Position } \\
\text { (SSTc2dJ...) }\end{array}$ & $\begin{array}{l}T_{\text {bol }} \\
(\mathrm{K}) \\
\end{array}$ & $\begin{array}{l}L_{\text {bol }} \\
\left(L_{\odot}\right) \\
\end{array}$ & $\alpha_{\mathrm{IR}}$ & $\begin{array}{c}M_{1 \mathrm{~mm}}{ }^{\mathrm{a}} \\
\left(M_{\odot}\right)\end{array}$ & Bolocam ID ${ }^{b}$ & Other Names \\
\hline \multicolumn{7}{|c|}{ Perseus } \\
\hline J032747.66+301204.6 & $662(15)$ & $2.80(0.05)$ & $-0.09(0.05)$ & $0.82(0.06)$ & Per-Bolo 24 & RNO $15^{\mathrm{c}}(1)$ \\
\hline $\mathrm{J} 034400.00+320154.1$ & $700(17)$ & $1.60(0.03)$ & $-0.34(0.05)$ & $0.40(0.04)$ & Per-Bolo $106^{*}$ & \\
\hline $\mathrm{J} 032900.00+312146.8$ & $856(26)$ & $0.33(0.01)$ & $-0.83(0.05)$ & $0.90(0.09)$ & Per-Bolo $40^{*}$ & $\mathrm{LkH} \alpha 353$ (2) \\
\hline J034109.13+314438.0 & $873(30)$ & $1.70(0.06)$ & $-0.72(0.05)$ & $0.16(0.02)$ & Per-Bolo 90 & IRAS $03380+3135$ \\
\hline J032856.64+311835.6 & $993(22)$ & $0.42(0.01)$ & $-0.78(0.05)$ & $0.10(0.02)$ & Per-Bolo 39* & ASR $120(3)$ \\
\hline J034548.27+322411.8 & $1096(23)$ & $5.00(0.09)$ & $-0.72(0.05)$ & $0.10(0.02)$ & Per-Bolo 120 & $\mathrm{LkH} \alpha 330$ (2) \\
\hline $\mathrm{J} 032917.66+312245.1$ & $1297(22)$ & $1.50(0.02)$ & $-0.77(0.05)$ & $0.19(0.02)$ & Per-Bolo 54* & $\mathrm{LkH} \alpha 270$ (2) \\
\hline \multicolumn{7}{|c|}{ Serpens } \\
\hline $\mathrm{J} 182900.00+003003.1$ & $806(5)$ & $0.19(0.01)$ & $-0.61(0.05)$ & $0.35(0.03)$ & Ser-Bolo 13* & \\
\hline $\mathrm{J} 183006.12+004233.9$ & $871(2)$ & $3.60(0.01)$ & $-0.42(0.05)$ & $0.17(0.01)$ & Ser-Bolo 33 & \\
\hline J182900.96+002931.6 & $892(2)$ & $1.20(0.01)$ & $-0.42(0.05)$ & $0.23(0.02)$ & Ser-Bolo $13^{*}$ & \\
\hline $\mathrm{J} 182901.32+002933.0$ & $892(6)$ & $0.36(0.01)$ & $-0.54(0.05)$ & $0.23(0.02)$ & Ser-Bolo 13* & \\
\hline J182901.68+002954.6 & $1158(3)$ & $1.80(0.01)$ & $-1.04(0.05)$ & $0.23(0.02)$ & Ser-Bolo $13^{*}$ & CoKu Ser G3 (4) \\
\hline $\mathrm{J} 182901.68+002946.5$ & $1212(5)$ & $0.70(0.01)$ & $-0.65(0.05)$ & $0.25(0.02)$ & Ser-Bolo $13^{*}$ & CoKu Ser G4 (4) \\
\hline \multicolumn{7}{|c|}{ Ophiuchus } \\
\hline J163151.96-245615.7 & $690(71)$ & $1.2(0.3)$ & $-0.17(0.05)$ & $0.05(0.01)$ & * & \\
\hline J162658.56-244531.6 & $824(14)$ & $1.3(0.1)$ & $-0.45(0.05)$ & $0.03(0.01)$ & & GY $186(5)$ \\
\hline J162624.00-241613.4 & $958(35)$ & $1.9(0.2)$ & $-0.71(0.05)$ & $0.04(0.01)$ & & \\
\hline J163945.36-240203.8 & $970(30)$ & $0.84(0.09)$ & $-0.73(0.05)$ & $0.03(0.01)$ & & \\
\hline $\mathrm{J} 162623.28-242100.7$ & $1080(100)$ & $2.0(0.4)$ & $-0.62(0.05)$ & $0.15(0.01)$ & Oph-Bolo $7^{*}$ & GY 20 (5) \\
\hline
\end{tabular}

Notes.

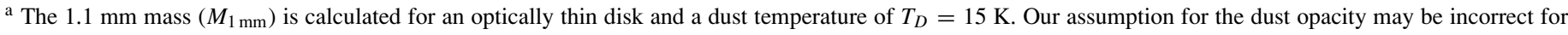
typical Class II disks, however, where grain growth is a possibility (e.g., Natta et al. 2007).

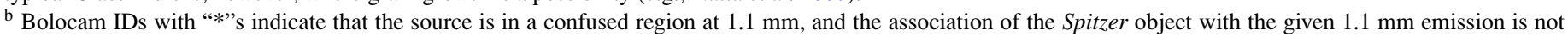
secure.

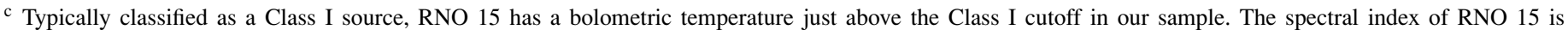
$\alpha_{\mathrm{IR}}=-0.09$, placing it in the "flat spectrum" category, or close to the Class I boundary.

References. (1) Cohen 1980; (2) Herbig \& Kameswara 1972; (3) Aspin et al. 1994; (4) Cohen \& Kuhi 1979; (5) Greene \& Young 1992.

\subsubsection{Ser-Bolo 33}

One noteworthy example of a Class II source associated with $1 \mathrm{~mm}$ emission is Ser-Bolo 33 (SSTc2dJ183006.12+004233.8) in Serpens (Figure 9), a very bright Spitzer source with $L_{\mathrm{bol}}=3.6 L_{\odot}, T_{\mathrm{bol}}=871 \mathrm{~K}$, and compact $1.1 \mathrm{~mm}$ emission centered on the Spitzer position. The spectral index, $\alpha_{\mathrm{IR}}=-0.42$, also places this object in Class II. Vieira et al. (2003) included this source in a sample of Herbig Ae/Be candidates, believed to be the intermediate mass Class II counterparts of low-mass $\mathrm{T}$ Tauri objects, although the spectral type is F3. The $1.1 \mathrm{~mm}$ mass calculated assuming an optically thin disk is $0.17 M_{\odot}$, approximately $10 \%$ of the stellar mass, $M_{*} \sim 1.3 M_{\odot}$, estimated from the measured effective temperature $\left(T_{\text {eff }} \sim 6300 \mathrm{~K}\right.$; Vieira et al. 2003) and an empirical $T_{\text {eff }}-M$ relation (Habets \& Heintze 1981).

\section{ALTERNATIVE CLASSIFICATIONS}

One advantage of such a large sample is that it allows us to define a more continuous evolutionary sequence than the standard classes that were appropriate for the smaller samples previously available. With this in mind, we divide the protostellar sources in each cloud into smaller $T_{\text {bol }}$ bins than those of the standard Class 0/I/II divisions. Average spectra for "early Class 0," "late Class 0," "early Class I," "late Class I," and Class II (see Table 1 for definitions) sources in Perseus and Serpens are shown in Figure 10. Error bars represent the $1 \sigma$ error in the mean, and average spectra are calculated as described in the previous section. The largest error bars are seen for the shortest wavelengths in the lowest $T_{\text {bol }}$ bins, where NIR fluxes vary significantly from source to source, likely depending on outflow opening angle and viewing geometry. Binning of $T_{\text {bol }}$ is based on general agreement of SEDs in a given bin, as determined by eye, and should not be interpreted as strict boundaries.

While there are significant similarities between the SEDs within each bin, individual source SEDS fill the continuum between the average spectra, and each average spectra lies within the $1 \sigma$ dispersion of neighboring $T_{\text {bol }}$ bins. The average spectra change as one would expect if an extincting envelope is gradually accreted or dispersed, with the protostar becoming more visible at short wavelengths. At all wavelengths except 24 and $70 \mu \mathrm{m}$, the flux rises or falls monotonically with increasing $T_{\text {bol }}$. For these intermediate wavelengths, the observed flux may rise initially as hotter dust close to the protostar is revealed, then fall as the mass of circumstellar material drops. Despite the relatively narrow bins, there is still a rather large change between "late Class 0" and "early Class I," particularly at $\lambda=3.6-24 \mu \mathrm{m}$; this transition may occur rapidly, or these wavelengths may be particularly sensitive to geometry.

\section{COMPARISON TO MODELS}

Even for infinitely well-sampled SEDs, spectrum shape is not necessarily directly correlated with age or degree of embeddedness. For example, viewing geometry can have a strong effect on SED shape. In three-dimensional radiative transfer models of Whitney et al. (2003), Robitaille et al. (2006), 

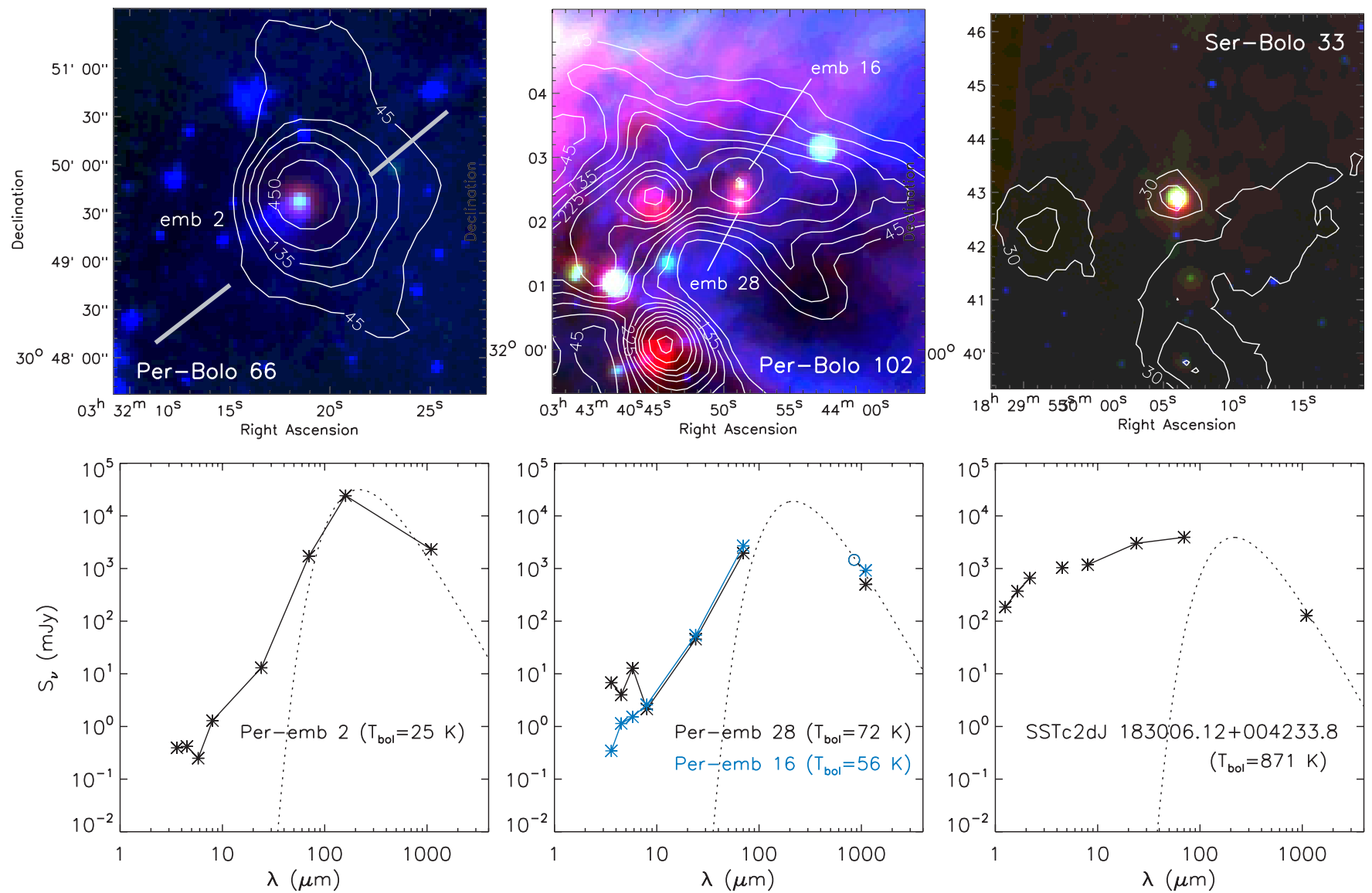

Figure 9. Three-color Spitzer images and SEDs of individual sources discussed in Section 5.2. Images are $(8,24,70 \mu \mathrm{m})$, unless otherwise noted, with $1.1 \mathrm{~mm}$ contours. Left: three-color $(3.6,24,70 \mu \mathrm{m})$ image of IRAS $03292+3039$ (Per-emb 2). Fan-shaped nebulosity is visible at $3.6 \mu \mathrm{m}$, most likely scattered light from a narrow outflow cavity in this deeply embedded Class 0 source. The thick lines indicate the approximate orientation of the larger-scale outflow (Jørgensen et al. 2006). Center: Per-Bolo 102, a new candidate binary Class 0/I source. Although the SEDs of the two embedded protostars look very similar at $\lambda \geqslant 8 \mu \mathrm{m}$, the southern source is brighter at shorter wavelengths, fainter at $2.7 \mathrm{~mm}$ (see the text), and has a slightly higher $T_{\text {bol }}$. Right: Ser-Bolo 33, a bright Class II object (Table 5). While this is clearly a more evolved source, it is massive enough that we detect $1.1 \mathrm{~mm}$ emission from a compact disk.

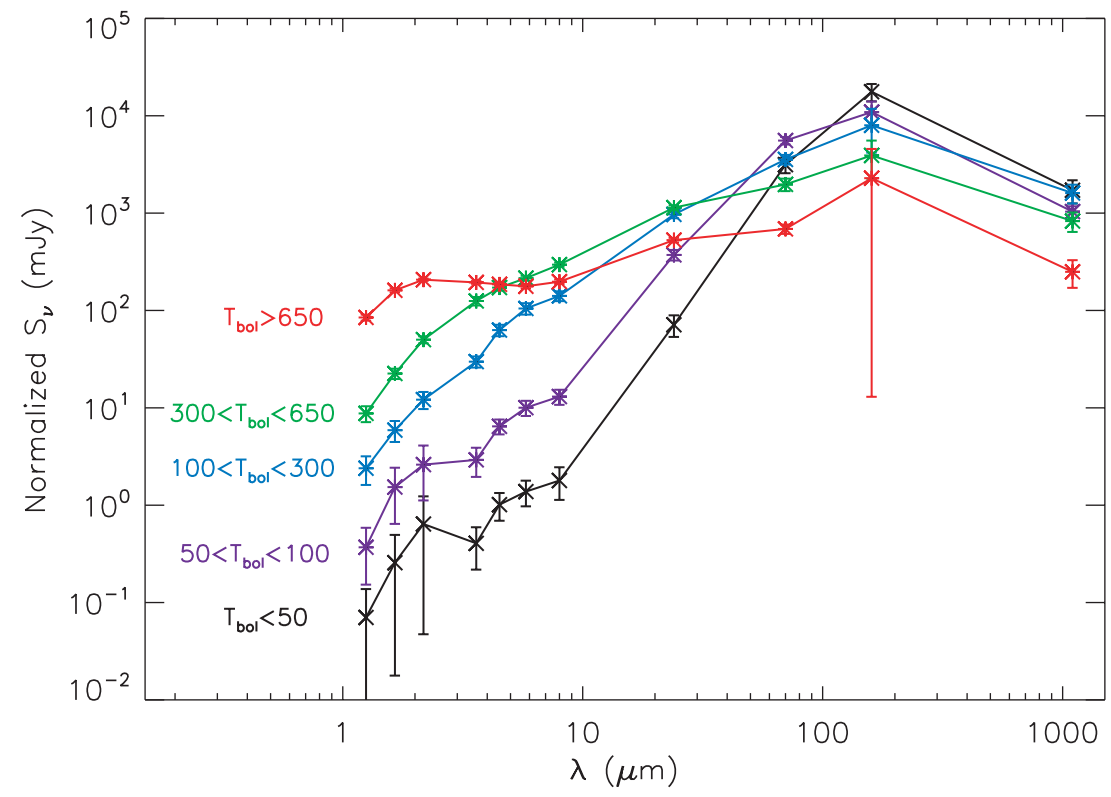

Figure 10. Average spectra in designated $T_{\text {bol }}$ bins (see Table 1), using sources from Perseus and Serpens. The average is calculated as described in Figure 8 and the text, and error bars represent the error in the mean. The progression in SED shape for increasing $T_{\text {bol }}$ is as expected if this represents a physical evolutionary sequence.

and Crapsi et al. (2008), Stage I and Stage II sources can have quite similar SEDs when viewed at the right inclination angle (e.g., when the observer's line-of-sight intersects the outflow opening angle of Stage I sources). Even Stage 0 sources can 

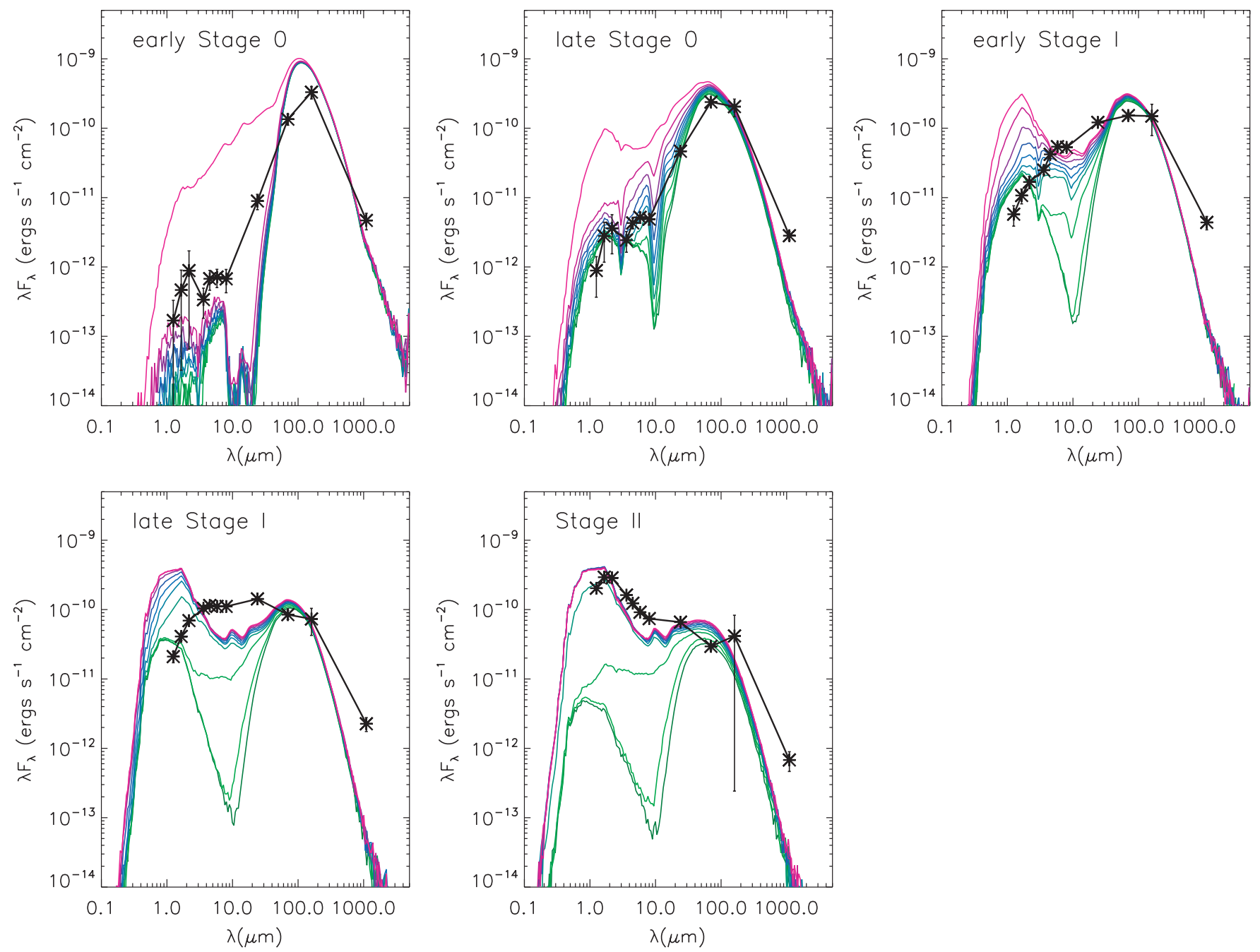

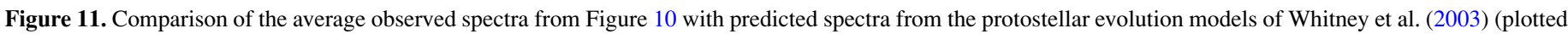

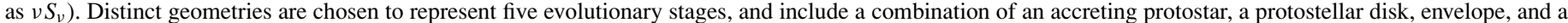

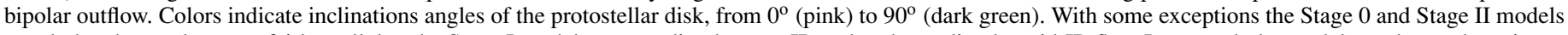

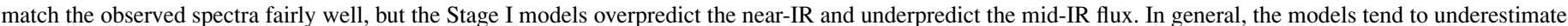
the millimeter flux and thus the mass of the protostellar envelope.

(A color version of this figure is available in the online journal.)

appear much warmer if we happen to be looking directly into the outflow cavity, although the probability of that occurring is small.

We compare our spectra to the results of protostellar models, which predict protostellar spectra based on source age, mass, accretion rate, etc., both to gain insight into the evolutionary state of sources and to evaluate the effectiveness of such models in matching observed sources. Rather than model each source individually, we compare the average spectra from Figure 10 with predicted spectra from Whitney et al. (2003) for "early Stage 0," "late Stage 0" sources, etc. in Figure 11.

Whitney et al. (2003) begin with a set geometry for each stage, including some combination of accreting protostar, flared protostellar disk, infalling envelope, bipolar outflow, and grain models for each region, then use radiative transfer modeling to predict protostellar spectra. Colors in Figure 11 correspond to inclination angles of the protostellar disk from $0^{\circ}$ (pink) to $90^{\circ}$ (green), where the outflow is perpendicular to the disk. Model envelope infall rates decline from $10^{-4} M_{\odot} \mathrm{yr}^{-1}$ in early Stage 0 to $10^{-6} M_{\odot} \mathrm{yr}^{-1}$ in late Stage I. Similarly, the disk radius and cavity opening angle increase and the cavity density decreases as one moves from early Stage 0 to late Stage I. Both models and average observed spectra (thick black lines) are scaled to a total luminosity of $1 L_{\odot}$.

The model early Stage 0 and observed early Class 0 spectra agree fairly well. The observed spectrum lies above most of the models at $\lambda \leqslant 24 \mu \mathrm{m}$, which could be explained by gaps in the inner envelope of some protostars, allowing short wavelength flux to escape (Jørgensen et al. 2005), or a wider outflow angle than the model $\left(5^{\circ}\right)$. The Whitney et al. (2003) models predict that a small fraction (approximately 1/10) of early Stage 0 sources will have spectra similar to Stage I, at very low inclination angles (looking down the outflow). The observed Class II spectrum is also consistent with at least the low inclination models; as we select for sources with $1.1 \mathrm{~mm}$ emission it is not unexpected that the observed millimeter point is higher than predicted by the models. For late Stage 0 the agreement is again pretty good, except that the model underpredicts the millimeter flux, and thus the mass of the protostellar envelope, a feature present in all spectra later than early Stage 0.

By contrast, the agreement between both Stage I models and the observed average spectra is quite poor. The models severely 


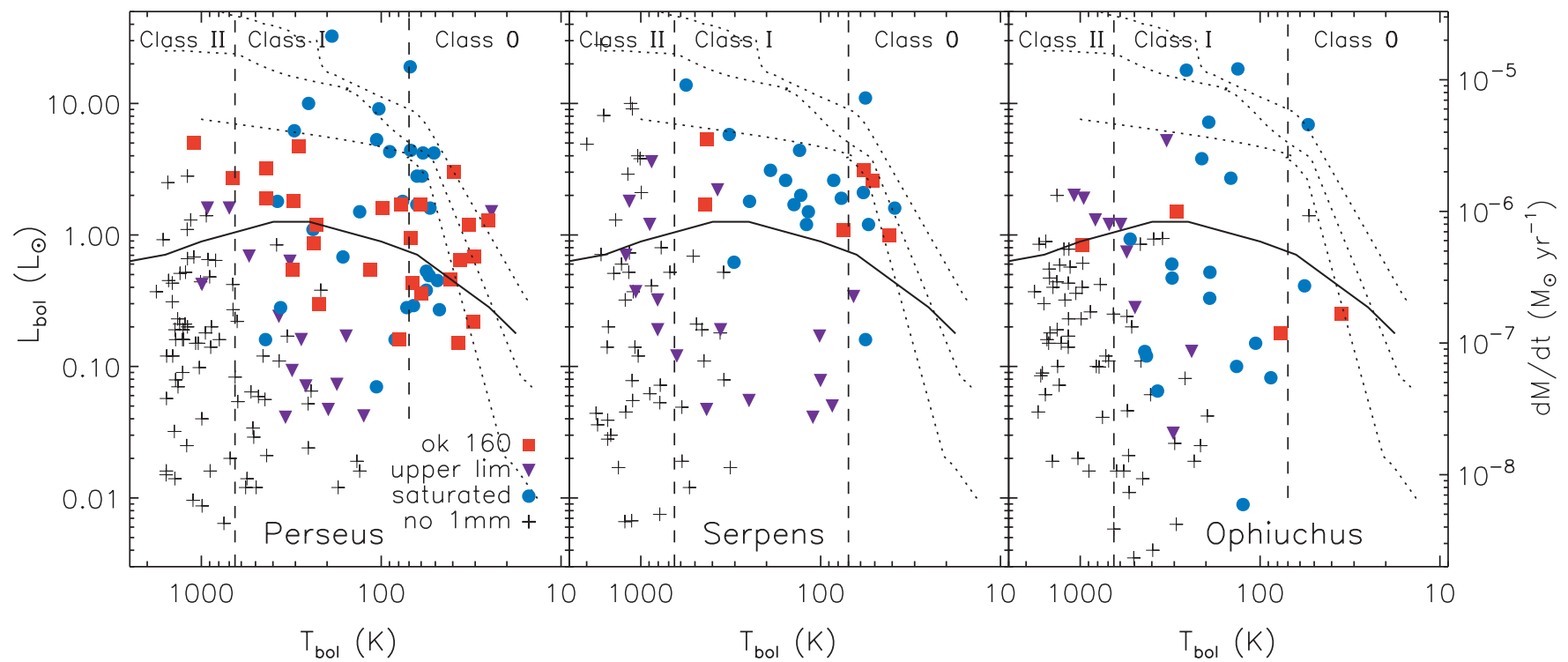

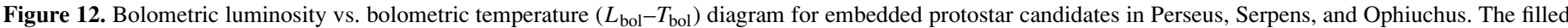

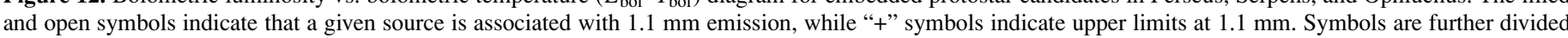

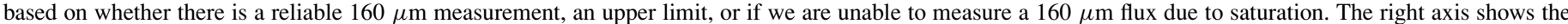

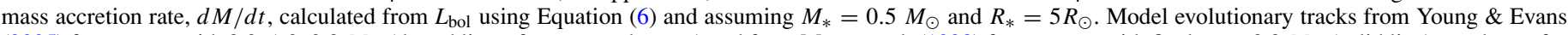

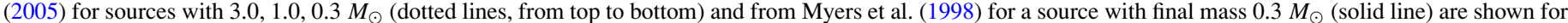
comparison. The large population of low-luminosity $\left(L_{\text {bol }}<0.1 L_{\odot}\right)$ Class I objects argues strongly for episodic accretion by Stage I.

(A color version of this figure is available in the online journal.)

overpredict the near-IR flux and underpredict the mid-IR flux. In fact, the characteristic double-peaked profile of the Stage I models is almost never seen in the observed spectra (Figure 7).

Indebetouw et al. (2006) demonstrate that envelopes with clumpy density distributions can increase the observed flux at $10 \mu \mathrm{m}$, with short wavelength emission escaping through low opacity regions between the clumps. This kind of clumpy distribution eliminates some of the "double-peaked" structure apparent in the Whitney et al. (2003) models, which assume a smooth density profile in the envelope and constant density in the outflow cavity, although the clumpy models also increase the flux at $<3 \mu \mathrm{m}$. Alternatively, the presence of a larger, thicker protostellar disk could flatten the Stage I spectra by absorbing near-IR flux and producing more mid-IR flux. A foreground cloud could also absorb near-IR flux, without producing any mid-IR flux.

It seems that we still do not understand how sources transition from Stage 0 to Stage I, at least in relation to these models. Whether it is the physical model that is unrealistic or how that physical geometry translates to the observed spectra is not clear. It is important to note that the same authors have developed a more complete grid of models (Robitaille et al. 2006); we compare here to the earlier models rather than fitting each average spectrum because we want an idea of the global agreement with evolutionary stage.

\section{PROTOSTELLAR EVOLUTION}

\subsection{Luminosity Evolution}

We examine the evolution of embedded protostars in more detail using the $L_{\mathrm{bol}}-T_{\mathrm{bol}}$ diagram, the protostellar equivalent of the H-R diagram (Myers et al. 1998). Here $T_{\text {bol }}$ is used as a measure of temperature rather than $T_{\text {eff }}$, which is not well characterized for embedded sources. As discussed in Myers et al. (1998), newly-formed protostars should begin at low $L_{\text {bol }}$ and $T_{\text {bol }}$, increasing in both $L_{\text {bol }}$ and $T_{\text {bol }}$ as accretion proceeds. If accretion abates or is otherwise halted, then $L_{\text {bol }}$ will decrease for steadily increasing $T_{\mathrm{bol}}$. Eventually, sources will move onto the main sequence, at $T_{\text {bol }} \gtrsim 3000 \mathrm{~K}$.

Figure 12 plots $L_{\text {bol }}$ versus $T_{\text {bol }}$ for the candidate embedded protostar samples in Perseus, Serpens, and Ophiuchus. Filled and open symbols are used for sources that are associated with $1.1 \mathrm{~mm}$ emission, while "+" symbols indicate sources with upper limits at $1.1 \mathrm{~mm} .{ }^{6}$ All sources detected at $1.1 \mathrm{~mm}$ are further divided according to whether they have reliable measured $160 \mu \mathrm{m}$ fluxes (squares), are saturated at $160 \mu \mathrm{m}$ (circles), or are not detected at $160 \mu \mathrm{m}$ (triangles). Given the discussion in the Appendix regarding the effect of missing $160 \mu \mathrm{m}$ fluxes on the calculation of $T_{\mathrm{bol}}$, circles would be expected to move up and to the right in this diagram for "cold" sources $\left(T_{\text {bol }} \lesssim 100 \mathrm{~K}\right)$, and down and to the left for "warm" sources $\left(T_{\text {bol }} \gtrsim 100 \mathrm{~K}\right)$, if $160 \mu \mathrm{m}$ fluxes were available.

While upper limits at $1.1 \mathrm{~mm}$ ("+" symbols) are expected for more evolved sources with $T_{\text {bol }} \gtrsim 500-600 \mathrm{~K}$, colder sources with no $1.1 \mathrm{~mm}$ detection may be either misclassified (e.g., background galaxies), or very low mass sources whose $1.1 \mathrm{~mm}$ flux is below our detection limit. Given our physical definition of Stage I $\left(M_{*} \gtrsim M_{\text {env }} ; M_{\text {env }}>0.1 M_{\odot}\right)$, embedded sources not detected at $1.1 \mathrm{~mm}$ must have stellar masses less than a few tenths of a solar mass, or have very little remaining envelope $\left(M_{*} \gg M_{\text {env }}\right)$.

Protostellar evolutionary tracks, which predict source properties as a function of age and mass, can easily be compared to our data using the $L_{\text {bol }}-T_{\text {bol }}$ diagram. Model tracks from Myers et al. (1998) (solid line; hereafter M98) and Young \& Evans (2005)

\footnotetext{
6 Note that although we plot sources with $1.1 \mathrm{~mm}$ upper limits here, our final embedded protostar samples (Tables 2-4) includes only sources with $1.1 \mathrm{~mm}$ detections.
} 
(dotted lines; hereafter YE05) are shown in Figure 12. YE05 adopt the standard inside-out collapse model of Shu (1977), and assume that no mass is lost in the formation process. From top to bottom, the YE05 models are for sources with masses of 3.0, 1.0, and $0.3 M_{\odot}$, assuming a constant accretion rate of $d M / d t=c_{s}^{3} / G$, where $c_{s}$ is the effective sound speed (Shu 1977). From the initial singular isothermal sphere, finite masses are achieved by truncating the outer radius of the envelope. A one-dimensional radiative transfer model (DUSTY) is used to calculate observational signatures $\left(L_{\mathrm{bol}}, T_{\mathrm{bol}}\right.$, etc.) from the accretion model.

Unlike YE05, M98 do not assume that the entire mass of the original core ends up in the final star, but rather that a significant fraction of the core mass is lost in the star formation process. The M98 model shown is for a source with initial core mass of $1.8 M_{\odot}$ and final stellar mass of $0.3 M_{\odot}$. M98 assume an accretion rate that is initially $d M / d t=c_{s}^{3} / G$, but falls off exponentially with time, designed to match the observed luminosity of pre-main-sequence stars at $T_{\mathrm{bol}} \gtrsim 3000 \mathrm{~K}$. Thus, the luminosity is significantly lower than the YE05 tracks at later times. Both evolutionary models assume an accreting central protostar, a circumstellar accretion disk, an extended envelope, and a contribution to the luminosity from gravitational contraction of the protostar. YE05 also include nuclear (deuterium) burning.

Ignoring for a moment the population of Class I sources in each cloud with $L_{\mathrm{bol}}$ values well below both models, the M98 model, for which a large fraction of the core mass is ejected or dispersed, is more consistent with the observed protostellar sources. The ratio of the final stellar mass to initial core mass for the M98 model shown $\left(f_{\text {eff }}=M_{\text {core }} / M_{\text {star }}=0.3 / 1.8=0.17\right)$ is smaller than the values $\left(f_{\text {eff }}=0.3 \pm 0.1\right)$ found by Alves et al. (2007) and Enoch et al. (2008) by comparing the shape of the core mass distribution to the initial mass function $\left(f_{\text {eff }}=0.3 \pm 0.1\right.$ and $f_{\text {eff }} \gtrsim 0.25$, respectively).

The slightly better match to the M98 model may be irrelevant, however, as neither a constant nor a steadily decreasing accretion rate is consistent with the observed protostellar populations in these clouds; many sources lie below all four model tracks. Our data confirm and exacerbate the "luminosity problem" noted by Kenyon et al. (1990) that Class I protostars in Taurus were observed to have lower $L_{\mathrm{bol}}$ values than expected based on the average mass accretion rate required to make a $1 M_{\odot}$ star.

In particular, the large population of Class I sources with low $L_{\mathrm{bol}}$ in each cloud is difficult to understand in relation to most existing protostellar evolutionary models. A general feature of such models is that the bolometric luminosity peaks in the Class I stage, a result that is true for constant accretion rates (YE05), decreasing rates (M98), and gravo-turbulent models (Froebrich et al. 2006). In contrast, we find quite similar mean luminosities for the Class 0 and Class I samples: $2.4 L_{\odot}$ and $2.2 L_{\odot}$, respectively. The standard deviation for both samples is large: $3.5 L_{\odot}$ and $4.7 L_{\odot}$ for Class 0 and Class I, respectively. The median luminosity of Class I sources is a factor of 3 lower than the mean, $0.7 L_{\odot}$.

\subsection{Episodic Accretion}

Given the above discussion and the large observed spread in $L_{\mathrm{bol}}$ of three orders of magnitude for Class I sources, much larger than the range in envelope masses, we conclude that mass accretion during the Class I stage is episodic. Class I sources with low $L_{\mathrm{bol}}$ can be explained by periods of relative quiescence when the bolometric luminosity, which is driven primarily by accretion luminosity, drops by at least a factor of 10. Conversely, Class I sources with high $L_{\text {bol }}$ values, e.g., those that form the upper envelope of the distribution and appear to be consistent with the YE05 models, would correspond to periods where the accretion rate is close to the "Shu accretion" value. Approximately $20 \%$ of the Class I sources have $L_{\text {bol }}<0.1 L_{\odot}$ (7/39 in Perseus, 5/25 in Serpens, and 5/25 in Ophiuchus), and could be considered candidate very low-luminosity objects (VeLLOs; Dunham et al. 2008; Di Francesco et al. 2007).

Episodic accretion is not an unreasonable solution; evidence for variable mass accretion and ejection is plentiful, including that based on modeling FU Orionis eruptions (e.g., Hartmann \& Kenyon 1985) and bow shocks in Herbig-Haro outflows (e.g., Reipurth \& Bally 2001). In traditional models of episodic accretion, infall from the envelope onto the disk is constant and accretion from the disk onto the protostar is episodic (e.g., Kenyon \& Hartmann 1995), for example due to gravitational instabilities in the disk (Vorobyov \& Basu 2006). Other scenarios such as the "spasmodic" infall model of Tassis \& Mouschovias (2005), where material is held up by the magnetic field at the inner edge of the envelope, are also plausible.

Although the observed distribution of $L_{\mathrm{bol}}$ for Class I sources argues strongly for episodic accretion, that is not the only possibility. One alternative is that the low- $L_{\text {bol }}$ Class I sources are simply very low mass objects, and we are somehow missing their low-mass Class 0 counterparts. Based on our sensitivity limits at $70,160 \mu \mathrm{m}$ and $1.1 \mathrm{~mm}$, we should be able to detect Class 0 sources with $L_{\text {bol }} \gtrsim 0.05 L_{\odot}$. The lower limit to the observed Class 0 luminosity, however, may not be the internal luminosity, but heating of the envelope by the interstellar radiation field (ISRF), which can contribute as much as $0.2-0.3 L_{\odot}$ to $L_{\text {bol }}$ for envelope masses of 1-3 $M_{\odot}$ (YE05). For the same reason, a Class 0 source with a very low mass accretion rate would not necessarily have a very low bolometric luminosity, making it difficult to determine if episodic accretion is already present at the Class 0 stage.

In Figure 13, we show the average spectrum of low-luminosity $\left(L_{\text {bol }}<0.2 L_{\odot}\right)$ Class I sources in Perseus and Serpens. On average, these sources have suppressed short wavelength points relative the $160 \mu \mathrm{m}$ and $1.1 \mathrm{~mm}$ fluxes, but are otherwise consistent in shape with the average Class I spectrum. ${ }^{7}$ The average low- $L_{\text {bol }}$ spectrum is consistent with low accretion luminosities (evident at short wavelengths) in sources with "normal" envelope masses, as expected if the low-luminosity objects are similar to other Class I sources but with lower mass accretion rates. The characteristics of low-luminosity protostars in Perseus, Serpens, and Ophiuchus are analyzed in Dunham et al. (2008).

\subsection{Mass Accretion Rates}

Assuming that the bolometric luminosity in Class 0 and Class I is due entirely to accretion, we can estimate the accretion rate from $L_{\mathrm{bol}}$ :

$$
\dot{M}=\frac{d M}{d t} \sim \frac{2 R_{*} L_{\mathrm{bol}}}{G M_{*}},
$$

where $R_{*}$ and $M_{*}$ are the radius and mass of the embedded protostar. For the following we assume $M_{*}=0.5 M_{\odot}$ and $R_{*}=5 R_{\odot}$, but accretion rates can be easily scaled for different values of the mass or radius. A mass of $0.5 M_{\odot}$ is consistent

\footnotetext{
7 Note that the average spectra are normalized by $1 / L_{\text {bol }}$, so on an absolute scale the low- $L_{\text {bol }}$ spectrum would be substantially fainter than the Class I spectrum.
} 


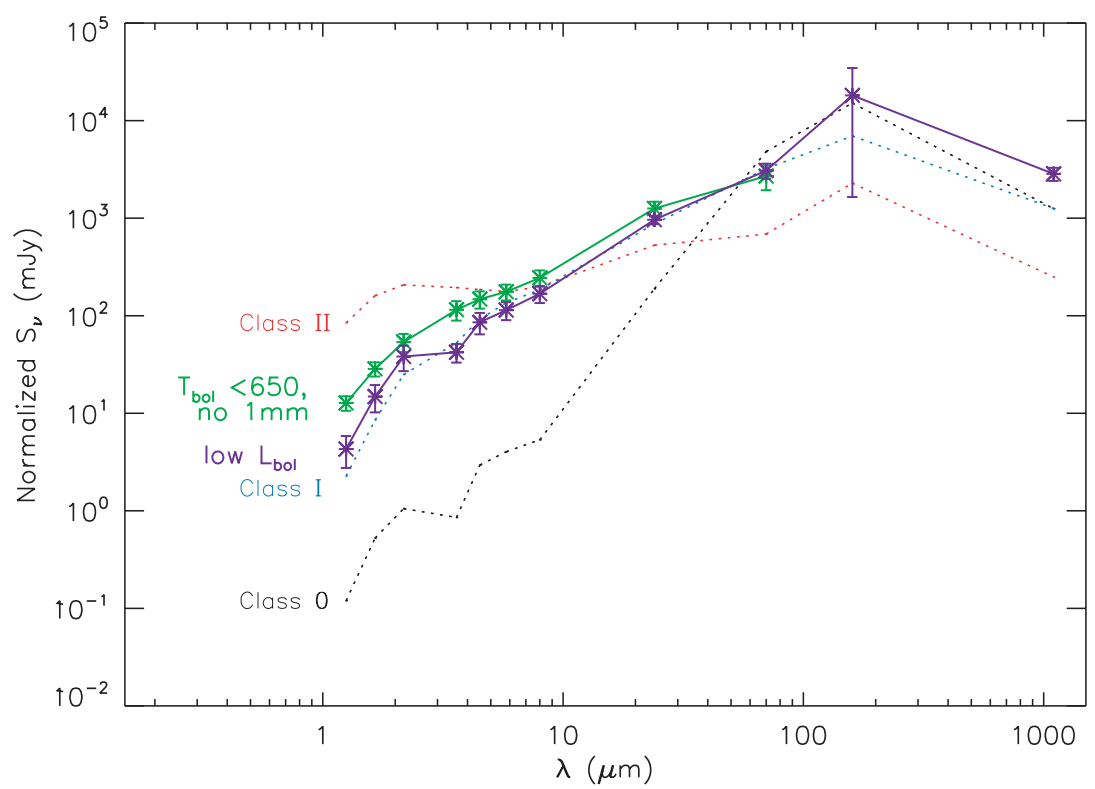

Figure 13. Average spectra of low-luminosity Class I sources and "nonenvelope Class I" sources (those that have $T_{\text {bol }}<650 \mathrm{~K}$ but no $1.1 \mathrm{~mm}$ emission). Average Class 0, I, and II spectra from Figure 8 are shown (dashed lines) for reference. The low- $L_{\mathrm{bol}}$ Class I sources have lower short wavelength points relative to the $160 \mu \mathrm{m}$ and $1.1 \mathrm{~mm}$ fluxes, as expected for low accretion rates, but are otherwise consistent with the average Class I spectrum. The average "nonenvelope Class I" spectrum is intermediate between the average Class I and Class II spectra, as expected if these sources represent an transitional stage in which there is little or no remaining envelope.

(A color version of this figure is available in the online journal.)

with the initial mass function (e.g., Chabrier 2003) and with average YSO masses (Merín et al. 2008; Spezzi et al. 2008); a radius of 3-5 $R_{\odot}$ is typical for pre-main-sequence models of low-mass sources (e.g., Palla \& Stahler 1991; Robitaille et al. 2006).

For comparison the "Shu accretion rate" of $c_{s}^{3} / G$, which is used in the YE05 models, is approximately $4 \times 10^{-6} M_{\odot} \mathrm{yr}^{-1}$ for $c_{s}=0.2 \mathrm{~km} \mathrm{~s}^{-1}$. The M98 model begins with $\dot{M} \sim 10^{-6} M_{\odot}$ $\mathrm{yr}^{-1}$, falling to $10^{-9} M_{\odot} \mathrm{yr}^{-1}$ by the time it reaches the main sequence. Making a $1 M_{\odot}$ star in $5.4 \times 10^{5} \mathrm{yr}$ requires an average accretion rate of approximately $2 \times 10^{-6} M_{\odot} \mathrm{yr}^{-1}$.

The mean luminosity of Class I sources corresponds to $\dot{M} \sim 1-2 \times 10^{-6} M_{\odot} \mathrm{yr}^{-1}$, not far from the average required to make a solar mass star. By contrast, the luminosity of the low$L_{\text {bol }}\left(L_{\text {bol }} \sim 0.1 L_{\odot}\right)$ Class I sources implies a mass accretion rate of only $7 \times 10^{-8} M_{\odot} \mathrm{yr}^{-1}$, substantially smaller than the Shu value or the average required to make a solar mass star. This result again suggests that either these sources will form very low mass stars $\left(M_{*} \lesssim 0.05 M_{\odot}\right)^{8}$, or that these sources are in a suppressed accretion phase of an episodic cycle. If we define "sub-Shu" accretion to be $\dot{M} \lesssim 10^{-6} M_{\odot} \mathrm{yr}^{-1}$, or $L_{\text {bol }} \lesssim 1 L_{\odot}$, then approximately $55 \%$ of Class I sources are observed to be in such a "sub-Shu" accretion state.

If the episodic accretion picture is correct there must be a population of sources with accretion rates much higher than the average; indeed, the highest observed luminosities $\left(L_{\text {bol }} \sim 10-30 L_{\odot}\right)$ imply $\dot{M} \sim 1-2 \times 10^{-5} M_{\odot} \mathrm{yr}^{-1}$, at least a factor of 5 higher than the Shu value or the average required to make a $1 M_{\odot}$ star. Approximately $5 \%$ of the Class I sources appear to be in such a "super-Shu" accretion rate $\left(L_{\text {bol }}>\right.$ $10 L_{\odot}$, or $\dot{M} \gtrsim 10^{-5} M_{\odot} \mathrm{yr}^{-1}$.

Unfortunately, it is very difficult to determine the duty cycle of the episodic accretion without detailed star formation and

\footnotetext{
8 Although this would require these sources to have a quite low core-to-star formation efficiency of $\lesssim 10 \%$, as only a few Class 0 sources have envelope masses less than $0.5 M_{\odot}$.
}

accretion models. The small fraction of sources observed to have very high accretion rates $\left(5 \%\right.$ with $\left.\dot{M} \gtrsim 10^{-5} M_{\odot} \mathrm{yr}^{-1}\right)$ suggests that periods of rapid accretion must be fairly short lived. Evans et al. (2009) employ a simple accretion model to estimate the time spent in accretion and quiescent phases, finding that half the mass of a $0.5 M_{\odot}$ star could be accreted during $7 \%$ of the Class I lifetime.

\subsection{Envelope Mass Evolution}

Protostellar evolution models also predict the evolution of envelope mass, $M_{\mathrm{env}}$, with $T_{\mathrm{bol}}$, as shown in Figure 14. Symbols are similar to Figure 12, with upper limits at $1.1 \mathrm{~mm}$ represented by arrows, and solid lines indicating the YE05 evolutionary tracks $\left(3.0,1.0\right.$, and $0.3 M_{\odot}$ top to bottom). Some of the higher $T_{\text {bol }}$ sources have high $1.1 \mathrm{~mm}$ upper limits because they lie in regions of extended or confused emission. Our determination that these sources are not actually associated with the $1.1 \mathrm{~mm}$ emission is based on visual examination of the images and SEDs.

Sources in all three clouds show a weak but consistent trend of decreasing $M_{\text {env }}$ with increasing $T_{\mathrm{bol}}$, as expected if the envelope is gradually depleted by accretion onto the protostar. This trend is not a result of including the $1.1 \mathrm{~mm}$ flux, from which the envelope mass is derived, in the calculation of $T_{\mathrm{bol}}$; the same trend is apparent even if the $1.1 \mathrm{~mm}$ point is excluded in the calculation of $T_{\mathrm{bol}}$.

Here, the YE05 model tracks fit the observed $M_{\mathrm{env}}-T_{\text {bol }}$ distribution quite well. Thus, a constant envelope infall rate reproduces the decrease in envelope mass with increasing $T_{\text {bol }}$, although it does not fit the evolution of $L_{\mathrm{bol}}$ with $T_{\mathrm{bol}}$ (Figure 12).

For standard episodic accretion models, where infall from the envelope is steady and the accretion rate from the disk to the protostar is the variable quantity (e.g., Kenyon \& Hartmann 1995), the envelope mass will steadily decrease with increasing $T_{\text {bol }}$ even for variable accretion. Note that accretion stops when the envelope mass, as defined by the outer radius, has been exhausted. In the context of the YE05 models, the spread of 


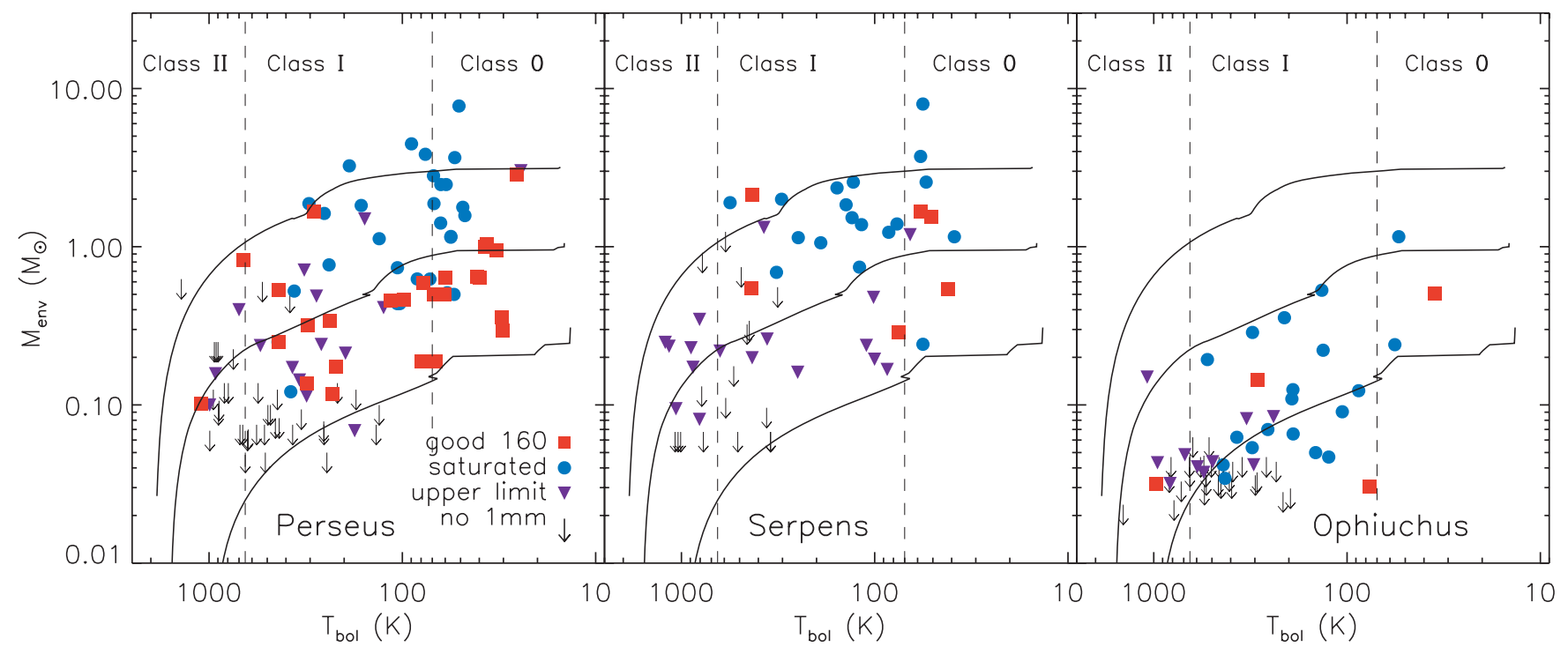

Figure 14. Envelope mass vs. bolometric temperature for embedded protostars in Perseus, Serpens, and Ophiuchus. The filled symbols indicate that a given protostar is associated with $1.1 \mathrm{~mm}$ emission, while sources with upper limits at $1.1 \mathrm{~mm}$ are plotted as arrows. The solid lines show the predictions of protostellar evolutionary models from Young \& Evans (2005) for sources of mass (from top to bottom) 3.0, 1.0, 0.3 $M_{\odot}$. The Young \& Evans (2005) models describe the evolution of envelope mass with $T_{\text {bol }}$ quite well, for sources of mass $M \sim 0.3-4 M_{\odot}$ in Perseus and Serpens, and $M \sim 0.1-1.5 M_{\odot}$ in Ophiuchus.

(A color version of this figure is available in the online journal.)

Table 6

Numbers of Protostars by Class and the Class 0 Lifetime

\begin{tabular}{lcccc}
\hline \hline Cloud & $N_{\text {Class } 0}$ & $N_{\text {Class I }}$ & $N_{\text {Class } 0} / N_{\text {Class I }}$ & $\begin{array}{c}\tau_{\text {Class } 0} \\
(\mathrm{yr})\end{array}$ \\
\hline Perseus & $27(27)$ & $39(41)$ & $0.70(0.66)$ & $2.2 \pm 0.4 \times 10^{5}$ \\
Serpens & $9(13)$ & $25(23)$ & $0.4(0.6)$ & $1.7_{-0.9}^{+0.5} \times 10^{5}$ \\
Ophiuchus & $3(4)$ & $25(24)$ & $0.12(0.17)$ & $0.7_{-0.5}^{+0.4} \times 10^{5}$ \\
Three-cloud sample & $39(44)$ & $89(88)$ & $0.44(0.50)$ & $1.72 \pm 0.25 \times 10^{5}$
\end{tabular}

Notes. Numbers of Class 0 and Class I sources are based on $T_{\text {bol }}$ classifications (Table 1) including the requirement that Class I sources be detected at $1.1 \mathrm{~mm}$. Numbers in parentheses indicate how the results change if we utilize a different method for calculating $T_{\text {bol }}$ (prismoidal vs. midpoint integration, see the Appendix). The Class 0 lifetime, $\tau_{\text {Class } 0}$, assumes that the entire embedded phase lasts for $5.4 \times 10^{5}$ years $\left(\tau_{\mathrm{emb}}=\tau_{\text {Class } 0}+\tau_{\text {Class I }}=5.4 \times 10^{5}\right.$ yr; Evans et al. 2009), and $N_{\text {Class } 0} /\left(N_{\text {Class } 0}+N_{\text {Class I }}\right)=\tau_{\text {Class } 0} / \tau_{\text {emb }}$. The lifetime is calculated using the average of the two $N_{\text {Class } 0} / N_{\text {Class I }}$ ratios given, and the uncertainty is from the difference between them added in quadrature with the $\sqrt{N}$ uncertainties from counting statistics.

$M_{\text {env }}$ as a function of $T_{\mathrm{bol}}$ suggests stellar masses in the range $M \sim 0.3-4 M_{\odot}$ in Perseus and Serpens, and $M \sim 0.1-1.5 M_{\odot}$ in Ophiuchus.

\section{LIFETIME OF THE CLASS 0 PHASE}

The length of time that sources spend in the Class 0 phase is an important diagnostic of protostellar evolution and how accretion rates evolve with time. If the rate of star formation in these clouds is steady in time (i.e., not occurring in bursts), and if Class 0Class I-Class II represents a true evolutionary sequence, then we can use the number of objects in consecutive evolutionary phases to estimate the relative lifetimes of those phases: $t_{1} / t_{2}=N_{1} / N_{2}$. As we ultimately calibrate lifetimes based on the lifetime of Class II disks $\left(\sim 2 \times 10^{6}\right.$ yr; Kenyon et al. 1990; Cieza et al. 2007; Spezzi et al. 2008), star formation must have been steady in time for at least the last $2 \mathrm{Myr}$. In addition, there can be no significant dependence of the evolutionary timescales on source mass.

It is unlikely that all of these assumptions hold in every star forming region. In fact, there is some observational evidence for mass-dependent evolution of dense cores (Hatchell et al. 2008), which could easily translate into mass-dependent evolution after protostar formation. We can, however, hope to mitigate the effects of any breakdowns in our assumptions by utilizing our large sample and averaging over three different environments.

In a companion paper (Enoch et al. 2008), we use a similar argument to derive the lifetime of the prestellar phase from the ratio of the number of starless cores to the total number of embedded protostars (Class $0+$ Class I). There we find that the lifetime of dense $\left(n \gtrsim 10^{4} \mathrm{~cm}^{-3}\right)$ prestellar cores is approximately equal to the lifetime of the embedded protostellar phase, or $2-5 \times 10^{5} \mathrm{yr}$, suggesting that such cores evolve dynamically over a few free-fall timescales.

The relative number of Class 0 and Class I sources in each cloud, and the Class 0 lifetime derived from that ratio, is given in Table 6. Given in parentheses are the values resulting from using the prismoidal, rather than midpoint method for determining $T_{\text {bol }}$; the Class 0 lifetime is based on an average of the two methods, and the difference between them, added in quadrature with $\sqrt{N}$ statistical uncertainties, yields an uncertainty for the lifetime.

There are approximately half as many Class 0 as Class I sources in both Perseus and Serpens $\left(N_{\text {Class } 0} / N_{\text {Class I }}=0.7\right.$ and 0.4 , respectively), suggesting that the Class 0 phase lasts 
Table 7

Effect of Extinction Corrections on Class Statistics

\begin{tabular}{lrccc}
\hline \hline Cloud & $N_{\text {Class 0 }}$ & $N_{\text {Class I }}$ & $N_{\text {Class 0 }} / N_{\text {Class I }}$ & $\begin{array}{c}\tau_{\text {Class 0 }} \\
(\mathrm{yr})\end{array}$ \\
\hline Perseus & 21 & 42 & 0.5 & $1.5 \times 10^{5}$ \\
Serpens & 4 & 26 & 0.15 & $0.6 \times 10^{5}$ \\
Ophiuchus & 3 & 20 & 0.15 & $0.6 \times 10^{5}$ \\
Three-cloud sample & 29 & 88 & 0.33 & $1.1 \times 10^{5}$ \\
\hline
\end{tabular}

Notes. Same as Table 6 but for classifications based on $T_{\text {bol }}^{\prime}$, the extinctioncorrected bolometric temperature. In addition, an embedded phase lifetime of $4.4 \times 10^{5} \mathrm{yr}$ is assumed, derived based on extinction-corrected classifications (Evans et al. 2009).

roughly half as long as the Class I phase. We adopt a total embedded phase lifetime of $t_{\mathrm{emb}}=t_{\mathrm{Cl} 0}+t_{\mathrm{Cl}} \sim 5.4 \times 10^{5} \mathrm{yr}$, derived based on the relative number of embedded protostars and Class II sources (Evans et al. 2009). Thus our measured ratios imply Class 0 lifetimes of $t_{\text {Class } 0} \sim 2.2 \times 10^{5} \mathrm{yr}$ in Perseus, and $1.7 \times 10^{5} \mathrm{yr}$ in Serpens. In Ophiuchus there are only three or four Class 0 sources, resulting in a ratio of $N_{\text {Class } 0} / N_{\text {Class I }}=0.1-0.2$ and $t_{\text {Class } 0} \sim 0.7 \times 10^{5} \mathrm{yr}$.

Taking all three clouds together, we find a lifetime for the Class 0 phase of $1.72 \pm 0.25 \times 10^{5} \mathrm{yr}$. This value is significantly longer than a number of previous estimates of $t_{\text {Class } 0} \sim 10^{4} \mathrm{yr}$, based both on the number of Class 0 sources in Ophiuchus (André \& Montmerle 1994), and on comparison to evolutionary models $\left(2-6 \times 10^{4} \mathrm{yr}\right.$; Froebrich et al. 2006). A short Class 0 lifetime is generally interpreted as evidence for a period of very rapid accretion early on in the evolution of protostars, causing them to quickly reach Stage I, at which point the accretion rate decreases significantly. Our results argue against such a rapid accretion phase. Although accretion may decrease somewhat in Class I (or become episodic, see Section 8), it appears unlikely that the average accretion rate drops by more than a factor of 2 from Class 0 to Class I, based on the relative lifetimes of the two phases. Similar mean luminosities for the Class 0 and Class I phases also argue against very high accretion rates in Class 0 .

Our derived Class 0 lifetime is similar to the results of Visser et al. (2002) for a sample of Lynds dark clouds ( $t_{\text {Class } 0} \sim 2 \times$ $10^{5} \mathrm{yr}$ ), and to the recent findings of Hatchell et al. (2007b) that the Class 0 lifetime in Perseus is similar to the Class I lifetime $\left(2.5-6.7 \times 10^{5} \mathrm{yr}\right.$ with $95 \%$ confidence $)$.

Our large, unbiased sample provides a distinct advantage over many other previous studies, which have necessarily relied on small samples or accumulated sources from a number of different surveys, wavelengths, and detection methods. For example, the Visser et al. (2002) Class 0 sample consists of seven sources, and the André \& Montmerle (1994) lifetime for Ophiuchus is based on one Class 0 object. Froebrich et al. (2006) note that their 50 Class 0/I sources are selected from a variety of surveys including NIR imaging of outflows, IRAS data, submillimeter and millimeter mapping, and radio continuum surveys, causing their source sample to be subject to strong selection effects. Within each cloud our surveys are very uniform, providing protostellar samples that are envelope mass limited and not biased by selection effects. For this reason, our estimated Class 0 lifetimes should be more robust than most previous measurements. The recent work by Hatchell et al. (2007b) comparing SCUBA $850 \mu \mathrm{m}$ maps and Spitzer c2d data of Perseus, with 34 Class 0 sources, is a notable exception.

Classifying sources based on $T_{\mathrm{bol}}^{\prime}$, calculated from photometry corrected for extinction (see Section 3.1.1), results in a somewhat smaller number of Class 0 sources, and a slightly shorter Class 0 lifetime. Table 7 gives the class statistics and corresponding Class 0 lifetime derived using the extinctioncorrected photometry. The three-cloud average Class 0 lifetime is shorter than when using observed photometry, by approximately $35 \%\left(t_{\text {class } 0}^{\prime}=1.1 \times 10^{5} \mathrm{yr}\right)$.

\subsection{Cloud-to-Cloud Differences: Ophiuchus}

The results in Ophiuchus are strikingly different from Perseus and Serpens, with nearly 10 times more Class I than Class 0 sources. Our results for Ophiuchus are similar to previously observed ratios of $N_{\text {Class } 0} / N_{\text {Class I }} \sim 1 / 10$ in that cloud (André $\&$ Montmerle 1994). The derived Class 0 lifetime, $t_{\text {Class } 0} \sim 7 \times$ $10^{4} \mathrm{yr}$, is still substantially longer than the very short $t_{\text {Class } 0} \sim$ $10^{4} \mathrm{yr}$ found by André \& Montmerle (1994), however.

There are two obvious, but conflicting, possible explanations for the smaller $N_{\text {Class 0 }} / N_{\text {Class I }}$ ratio in Ophiuchus. First, the star formation rate may be temporally variable in Ophiuchus. Visser et al. (2002) suggest that a burst of star formation approximately $10^{5} \mathrm{yr}$ ago is responsible for the large ratio of Class I to Class 0 sources. Alternatively, the Class 0 phase may be much shorter in Ophiuchus than in the other two clouds due to higher accretion rates at early times. Were this the case, however, we would expect the mean luminosity to be significantly higher for the Class 0 sources than for the Class I sources, which is not observed. While a burst of star formation seems the more likely explanation, accretion rates, and thus lifetimes, could conceivably depend on cloud environmental factors such as mean density or turbulent velocity.

On the other hand, the observed number of Class 0 sources in Ophiuchus may be biased by the dearth of $160 \mu \mathrm{m}$ flux measurements in that cloud. As discussed in the Appendix, bolometric temperatures are likely overestimated for sources without a $160 \mu \mathrm{m}$ fluxes, which may cause up to $50 \%$ of Class 0 sources to be classified as Class I. The lack of $160 \mu \mathrm{m}$ fluxes is especially problematic in Ophiuchus, where the majority of sources are either saturated at $160 \mu \mathrm{m}$ or in regions of poor coverage, and may be partially responsible for the small number of observed Class 0 sources.

\subsection{Limitations on the Class I Sample}

Recall that we only include sources in our Class I sample if they are detected at $1.1 \mathrm{~mm}$. This criterion excludes 24 sources from the original candidate protostar sample in Perseus with $70<T_{\text {bol }}<650 \mathrm{~K}, 18$ sources in Serpens, and 36 in Ophiuchus. The average spectrum of these "nonenvelope Class I" sources is shown in Figure 13 (Perseus and Serpens only), and appears to be intermediate between the Class I and Class II averages. Furthermore, the mean $T_{\text {bol }}$ values of the nonenvelope Class Is (570 K in Perseus, $520 \mathrm{~K}$ in Serpens, and $500 \mathrm{~K}$ in Ophiuchus) confirm that they fall at the warm end of the Class I distribution. While it is possible that we are simply not detecting the envelopes of these sources (e.g., if $M_{\text {env }} \lesssim 0.1 M_{\odot}$ or they are very diffuse), we suggest that the transition from Stage I (protostars that retain an envelope) to Stage II (premain-sequence stars with no envelope) occurs closer to $T_{\mathrm{bol}}=$ $400-500 \mathrm{~K}$ than the standard $T_{\text {bol }}=650 \mathrm{~K}$, for these data.

When we add the "nonenvelope Class I" sources to our Class I samples, dropping the requirement that they be detected at $1.1 \mathrm{~mm}$, the Class 0 to Class I ratio becomes 0.5 in Perseus, 0.3 in Serpens, and 0.07 in Ophiuchus. This represents a 25\%$30 \%$ decrease in the Class 0 lifetime for each cloud. On the other hand, Crapsi et al. (2008) suggest based on protostellar models 
Table 8

Lifetimes for Narrow $T_{\text {bol }}$ Bins

\begin{tabular}{|c|c|c|c|c|c|c|c|}
\hline Phase & $\begin{array}{c}T_{\text {bol }} \text { Range } \\
(\mathrm{K})\end{array}$ & $N($ Per $)$ & $N($ Ser $)$ & $N(\mathrm{Oph})$ & $\begin{array}{c}N(\text { total }) / \\
N(\text { emb,tot })\end{array}$ & $\begin{array}{c}\text { Mean } T_{\text {bol }} \\
(\mathrm{K})\end{array}$ & $\begin{array}{c}\text { Lifetime } \\
(\mathrm{yr})\end{array}$ \\
\hline Early Class 0 & $T<50$ & 11 & 2 & 1 & $14 / 128=0.11$ & 40 & $0.6 \times 10^{5}$ \\
\hline Early Class I & $100<T<300$ & 19 & 12 & 12 & $43 / 128=0.34$ & 180 & $1.8 \times 10^{5}$ \\
\hline Late Class I & $300<T<650$ & 13 & 9 & 11 & $33 / 128=0.26$ & 400 & $1.4 \times 10^{5}$ \\
\hline
\end{tabular}

Notes. For each $T_{\text {bol }}$ bin, $N$ (total) is the total number of sources in all three clouds; $N$ (emb,tot) is the total number of embedded protostars in the three-cloud sample (128). Only sources detected at $1.1 \mathrm{~mm}$ are included. The mean $T_{\text {bol }}$ of protostars in a given $T_{\text {bol }}$ range is also given. Lifetimes are calculated from $N$ (total) $/ N(\mathrm{emb}$, tot $) \times 5.4 \times 10^{5}$ yr. Cloud-to-cloud variations are typically $0.2-0.3 \times 10^{5} \mathrm{yr}$.

that up to half of sources observationally classified as Class I may actually be Stage II source with disk inclinations $\gtrsim 65^{\circ}$. Our "nonenvelope Class I" objects may be just such sources; if the Crapsi et al. (2008) picture is correct, the observed number of Class I sources likely represents an upper limit to the true number of Stage I protostars.

\subsection{Evolution of $T_{\mathrm{bol}}$ with Time}

In Table 8, we give the number of sources in each cloud in our narrow $T_{\text {bol }}$ bins ("early Class 0," "late Class 0," etc.; Section 6). The ratio of the total number in each bin, summed over the three clouds, to the total number of embedded protostars is used to derive a lifetime for each $T_{\text {bol }}$ range. The mean $T_{\text {bol }}$ within that range is also given.

With the exception of "early Class 0 ," there is no evidence for a difference in the number of sources in each $T_{\mathrm{bol}}$ bin, and little difference in the derived lifetimes. Notably, $75 \%$ of the embedded phase lifetime has elapsed by the time the bolometric temperature reaches $300 \mathrm{~K}$, and the mean $T_{\text {bol }}$ in "Late Class I" $(400 \mathrm{~K})$ is skewed toward the lower temperature end of the bin. These features support our earlier suggestion that the dividing line between Stage I and Stage II is probably closer to $T_{\text {bol }}=400-500 \mathrm{~K}$ than to $650 \mathrm{~K} . T_{\text {bol }}=50 \mathrm{~K}$ is reached at quite early times, which is not unexpected if the temperature scale starts at approximately $10 \mathrm{~K}$ with starless cores.

With lifetime measurements in several $T_{\text {bol }}$ intervals, together with our previous conclusion that the average accretion rate is approximately constant through the embedded phase, we can empirically characterize the evolution of $T_{\mathrm{bol}}$ with time. Figure 15 plots the evolution of the bolometric temperature with time, based on values from Table 8 . The relationship is clearly nonlinear; given the small number of observed points, we fit a simple function to $T_{\mathrm{bol}}$ as a function of time:

$$
T_{\mathrm{bol}}=25 \mathrm{~K}+C\left(\frac{t}{10^{5} \mathrm{yr}}\right)^{n}
$$

where $C$ is constant and we choose $25 \mathrm{~K}$ as the temperature at $t=0$ because that is the minimum observed $T_{\text {bol }}$ in Class 0 . The best fit is for $C=51 \mathrm{~K}$ and $T_{\mathrm{bol}} \propto t^{1.8}$, indicating a fairly steep evolution with time. The dependence on time must flatten out significantly after the embedded phase, however, to match the lifetime for Class II (the dashed line in Figure 15).

\section{CONCLUSIONS}

Utilizing large-scale $1.1 \mathrm{~mm}$ surveys (Enoch et al. 2006, 2007; Young et al. 2006) together with Spitzer IRAC and MIPS maps from the c2d Legacy program (Evans et al. 2003), we have constructed an unbiased census of deeply embedded protostars in the Perseus, Serpens, and Ophiuchus molecular clouds. Our sample includes a total of 39 Class 0 sources and 89 Class I sources, with approximate detection limits of $M_{\text {env }} \gtrsim 0.1 M_{\odot}$ and $L_{\text {bol }} \gtrsim 0.05 L_{\odot}$ for the envelope mass and bolometric luminosity, respectively. We also detect a few Class II and Herbig $\mathrm{Ae} / \mathrm{Be}$ candidates at $1.1 \mathrm{~mm}$, most likely evidence of fairly massive proto-planetary disks.

Bolometric luminosities, temperatures, and envelope masses are calculated for the candidate Class 0 and Class I sources in each cloud. We compare protostellar classification methods, concluding that, for deeply embedded sources, the bolometric temperature $T_{\mathrm{bol}}$ is a better measure of evolutionary state than the near- to mid-IR spectral index, $\alpha_{\mathrm{IR}}$.

We also explore classifying sources into "early Class 0," "late Class I," etc., based on dividing them into narrower $T_{\text {bol }}$ bins. Average observed spectra in these bins are compared to model predictions from Whitney et al. (2003) for "early Stage 0," "late Stage 0," etc. In a broad sense the Stage 0 and Stage II models match the observed spectra fairly well. The agreement with both Stage I models is quite poor, however, as the models severely overpredict the near-IR and underpredict the mid-IR flux at this stage, displaying a double-peaked SED that is rarely observed.

Observed source properties are compared to protostellar evolutionary models using the bolometric temperature-luminosity ( $\left.L_{\mathrm{bol}}-T_{\mathrm{bol}}\right)$ diagram, the protostellar equivalent of the H-R diagram (Myers et al. 1998). Neither models with a constant mass accretion rate (Young \& Evans 2005) nor those with an exponentially decreasing rate (Myers et al. 1998) fit the observed sources.

In particular, there is a large population of low-luminosity Class I sources that aggravate the previously noted "luminosity problem" for embedded protostars (e.g., Kenyon et al. 1990). We interpret this result as evidence for episodic accretion beginning at least by the Class I phase, and possibly earlier. More than $50 \%$ of Class I sources are inferred to have "sub-Shu" mass accretion rates $\left(\dot{M} \lesssim 10^{-6} M_{\odot} \mathrm{yr}^{-1}\right.$, corresponding to $\left.L_{\text {bol }} \lesssim 1 L_{\odot}\right)$, and approximately $20 \%$ have $\dot{M} \lesssim 10^{-7} M_{\odot} \mathrm{yr}^{-1}$. To build up of order a solar mass in $5.4 \times 10^{5} \mathrm{yr}$, such sources must also have periods of "super-Shu" accretion $\left(\dot{M} \gtrsim 10^{-5} L_{\odot} \mathrm{yr}^{-1}\right)$. Few very high luminosity sources are observed (5\%), suggesting that such rapid accretion periods must be short lived. An important caveat to this analysis is that we may sometimes underestimate the luminosity due to missing 70 or $160 \mu \mathrm{m}$ photometry.

Finally, the relative number of Class 0 and Class I sources is used to estimate the lifetime of the Class 0 phases. There are approximately half as many Class 0 as Class I sources in the three-cloud sample, implying an average Class 0 lifetime of $1.7 \pm 0.3 \times 10^{5} \mathrm{yr}\left(1.1 \times 10^{5} \mathrm{yr}\right.$ when approximate extinction 


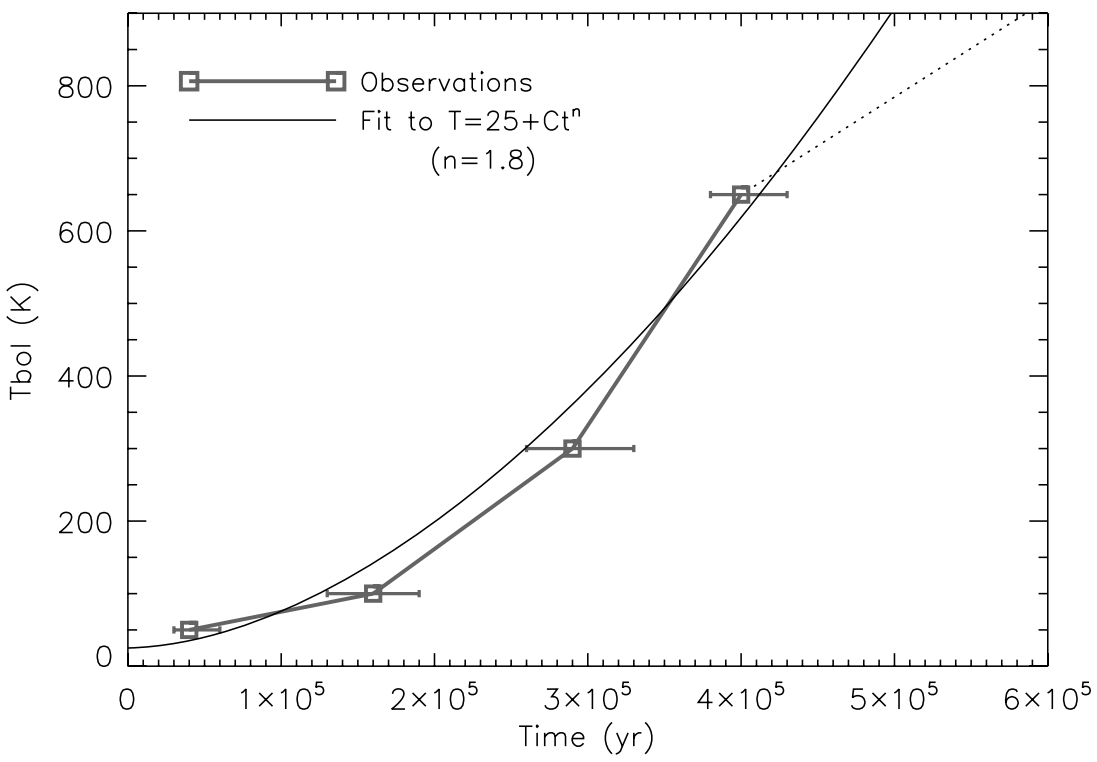

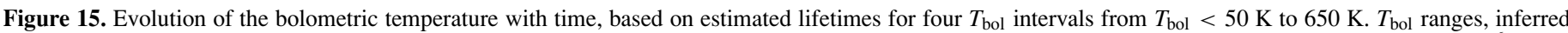

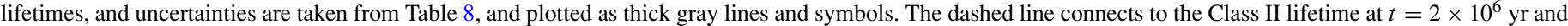
$T_{\text {bol }}=2800 \mathrm{~K}$. We fit a simple function to $T_{\text {bol }}$ vs. time: $T_{\text {bol }}=25 \mathrm{~K}+C\left(t / 10^{5} \mathrm{yr}\right)^{n}$; the best fit is for $T_{\text {bol }} \propto t^{1.8}$.

corrections are applied). This lifetime rules out drastic changes in the mass accretion rate from Class 0 to Class I, particularly extremely rapid accretion in Class 0 . In Ophiuchus, the fraction of Class 0 sources is much smaller. While this difference could be due to the lack of $160 \mu \mathrm{m}$ flux measurements in Ophiuchus, it may be that either the Class 0 phase is shorter in that cloud $\left(0.7 \times 10^{5} \mathrm{yr}\right)$, or that a burst of star formation is responsible for the large population of Class I objects (e.g., as suggested by Visser et al. 2002).

Altogether, the large variation in Class I luminosities, similar mean luminosities for the Class 0 and Class I phases, and not dramatically different numbers of Class 0 and Class I sources (at least in Perseus and Serpens), suggests a consistent picture of nearly constant average mass accretion rate through the entire embedded phase (Stage 0 and Stage I), with highly variable episodic accretion turning on by at least early Stage I, and possibly sooner. Understanding embedded protostellar structure and evolution well enough to reproduce the observations with detailed models of spectra and evolution presents an ongoing challenge.

The authors thank J. Hatchell and Y. Shirley, and the anonymous referee for comments and suggestions that helped to improve this paper, and M. Dunham for many fruitful discussions. We are grateful to B. Whitney for sharing the protostellar evolutionary model data used here. We thank the Lorentz Center in Leiden for hosting meetings that contributed to this paper. Part of the work was done while in residence at the Kavli Institute for Theoretical Physics in Santa Barbara, California. Support for this work, part of the Spitzer Legacy Science Program, was provided by NASA through contracts 1224608 and 1230782 issued by the Jet Propulsion Laboratory, California Institute of Technology, under NASA contract 1407. Additional support was provided by NASA through the Spitzer Space Telescope Fellowship Program and obtained from NASA Origins Grants NNG04GG24G and NNX07AJ72G to the University of Texas at Austin. Support for the development of Bolocam was provided by NSF grants AST-9980846 and AST-0206158.

\section{APPENDIX}

\section{CALCULATING THE BOLOMETRIC LUMINOSITY AND TEMPERATURE}

Determination of the bolometric temperature and luminosity of any given source can depend strongly on the method used to approximate the integrations over frequency in Equations (1)(3). As $S_{v}$ is sampled at a finite number of frequencies (usually 6-10 here), the SED must be interpolated over the intermediate frequencies. Here we use two different methods; the first (midpoint) method utilizes a simple linear interpolation for the midpoint flux, while the second (prismoidal) method calculates a color temperature for each pair of flux points, and uses a modified blackbody based on that color temperature to estimate the midpoint flux density. In both cases the SED is extrapolated from the longest wavelength flux using $S_{v} \propto v^{2}$, and flux upper limits are removed from the fit (i.e., we interpolate over them). Examples of the midpoint and prismoidal interpolations are shown in Figure 16 for two sources with Class 0 like (left) and Class I like (right) SEDs.

Figure 17 compares the results of using the midpoint and prismoidal methods to calculate $L_{\mathrm{bol}}$ and $T_{\mathrm{bol}}$ for the embedded protostar sample in Perseus. The midpoint and prismoidal $T_{\text {bol }}$ values agree fairly well, usually to within $20 \%$, with no strong systematic bias. There is some tendency for the prismoidal $T_{\mathrm{bol}}$ to be lower for colder sources. On the other hand, $L_{\text {bol }}$ calculated by the prismoidal method is generally higher than the midpoint $L_{\text {bol }}$.

To further test this behavior, we plot in Figure 18 the fractional errors due solely to finite sampling, for $L_{\text {bol }}$ and $T_{\text {bol }}$ calculated from input blackbody spectra. Blackbody sources with input $T_{\text {bol }}$ from 5 to $5000 \mathrm{~K}$ are sampled at the observed wavelengths $(\lambda=1.25,1.65,2.17,3.6,4.5,5.8,8,24,70,160,1100 \mu \mathrm{m})$, and the bolometric temperature and luminosity calculated by the midpoint (left) and prismoidal (right) methods. Both $L_{\mathrm{bol}}$ and $T_{\text {bol }}$ have typical errors of approximately $20 \%$, and the measured $T_{\text {bol }}$ is consistently overestimated by approximately $20 \%$. The midpoint $T_{\text {bol }}$ is more variable but somewhat more 

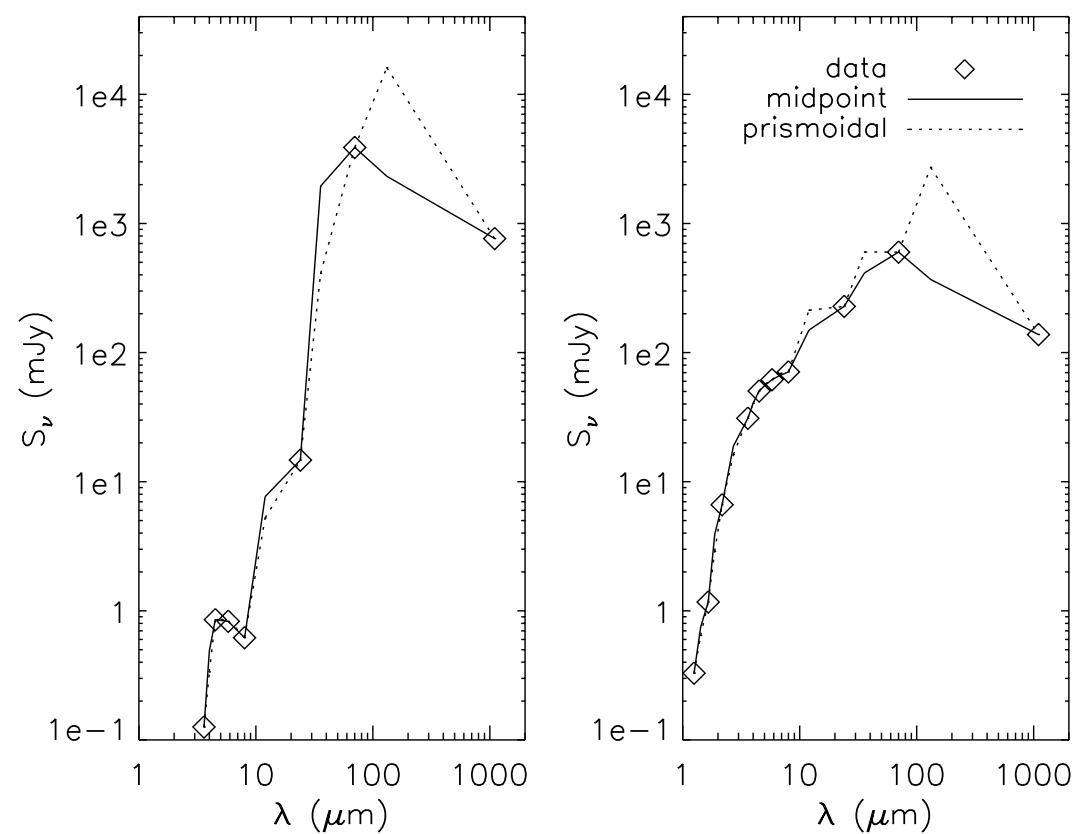

Figure 16. Examples of the interpolated midpoints used for the integration of the SED in the midpoint (solid line) and prismoidal (dashed line) methods. The observed data points are overlaid as diamonds. A typical Class 0 source is shown on the left, and a Class I source on the right; both sources shown are lacking data at $160 \mu \mathrm{m}$. After the midpoint is calculated, the integration for both methods is done in linear $\nu S_{v}$ space. The prismoidal method provides a good approximation of the dust emission peak for cold sources (left), but probably overestimates the long-wavelength flux for warmer, flatter SED sources (right), resulting in a bias toward lower $T_{\text {bol }}$.
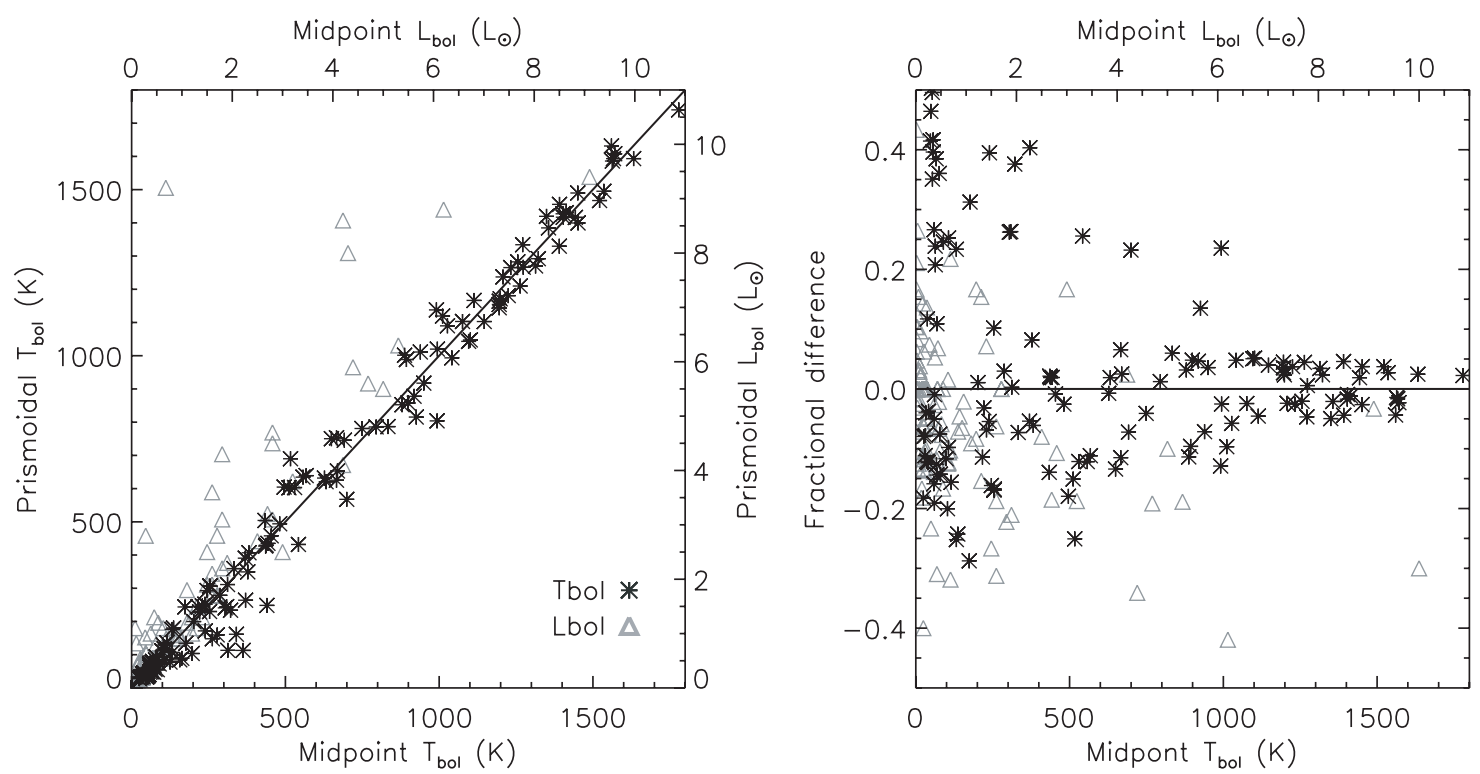

Figure 17. Comparison of the midpoint and prismoidal methods for calculating $L_{\mathrm{bol}}$ and $T_{\mathrm{bol}}$, for sources in Perseus. Left: midpoint and prismoidal $T_{\mathrm{bol}}$ (black asterisks) and $L_{\mathrm{bol}}$ (triangles) values are plotted for all candidate embedded protostars in Perseus. Right: fractional difference between the midpoint and prismoidal methods, plotted as (midpoint-prismoidal)/prismoidal. The two methods generally agree to within $20 \%$ for $T_{\text {bol }}$ but $L_{\text {bol }}$ calculated by the prismoidal method is higher than the midpoint $L_{\text {bol }}$ by as much as $100 \%$. There is some tendency for the prismoidal $T_{\text {bol }}$ to be lower for colder sources.

accurate than the prismoidal $T_{\text {bol }}$, while $L_{\text {bol }}$ is somewhat less accurate. Of course, the blackbody spectra tested here are not necessarily representative of the more complicated observed SEDs. Based on Figures 17 and 18 and on examinations of the midpoint and prismoidal fits to observed SEDs, from which we find that the prismoidal method often provides a poor fit to "flatter" SEDs at long wavelengths (Figure 16), we use the midpoint method throughout this paper. Differences between the midpoint and prismoidal methods are often used as a measure of uncertainty.

In general, measured $160 \mu \mathrm{m}$ fluxes are uncertain by up to a factor of 2, due to unquantified saturation effects and calibration uncertainties, and in many cases we are unable to measure a $160 \mu \mathrm{m}$ flux at all. In addition, the "pointsource" $160 \mu \mathrm{m}$ fluxes of extended sources will likely be underestimated, because they are measured with a PSF fit rather than aperture photometry. Missing or severely underestimated fluxes at $160 \mu \mathrm{m}$ can significantly affect both $L_{\mathrm{bol}}$ and $T_{\text {bol }}$ for cold sources that peak near $100 \mu \mathrm{m}$. As we are primarily interested in the coldest sources, we attempt here to quantify the effects of missing or underestimated $160 \mu$ m flux. One way of approaching this problem is to examine sources for which we have additional information near the peak of the SED, e.g., from IRAS or SHARC $350 \mu \mathrm{m}$ observations (Wu et al. 2007). 

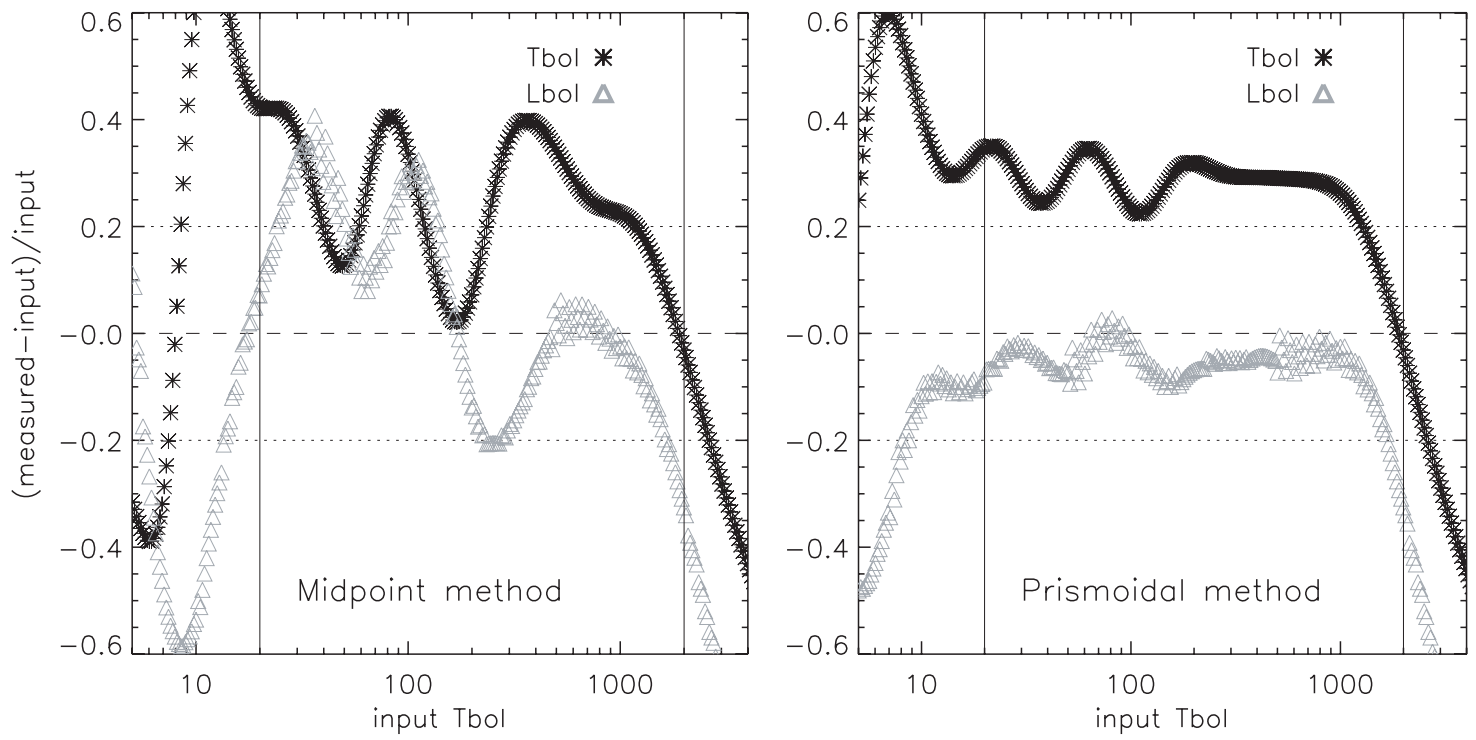

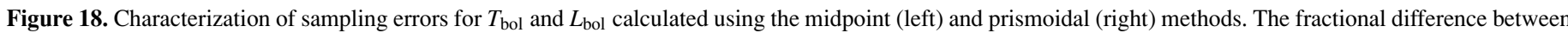

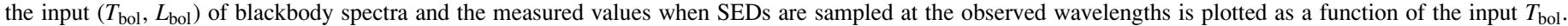

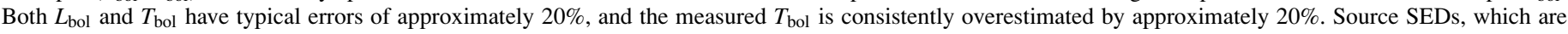
considerably more complex than the blackbodies tested here, may behave differently.
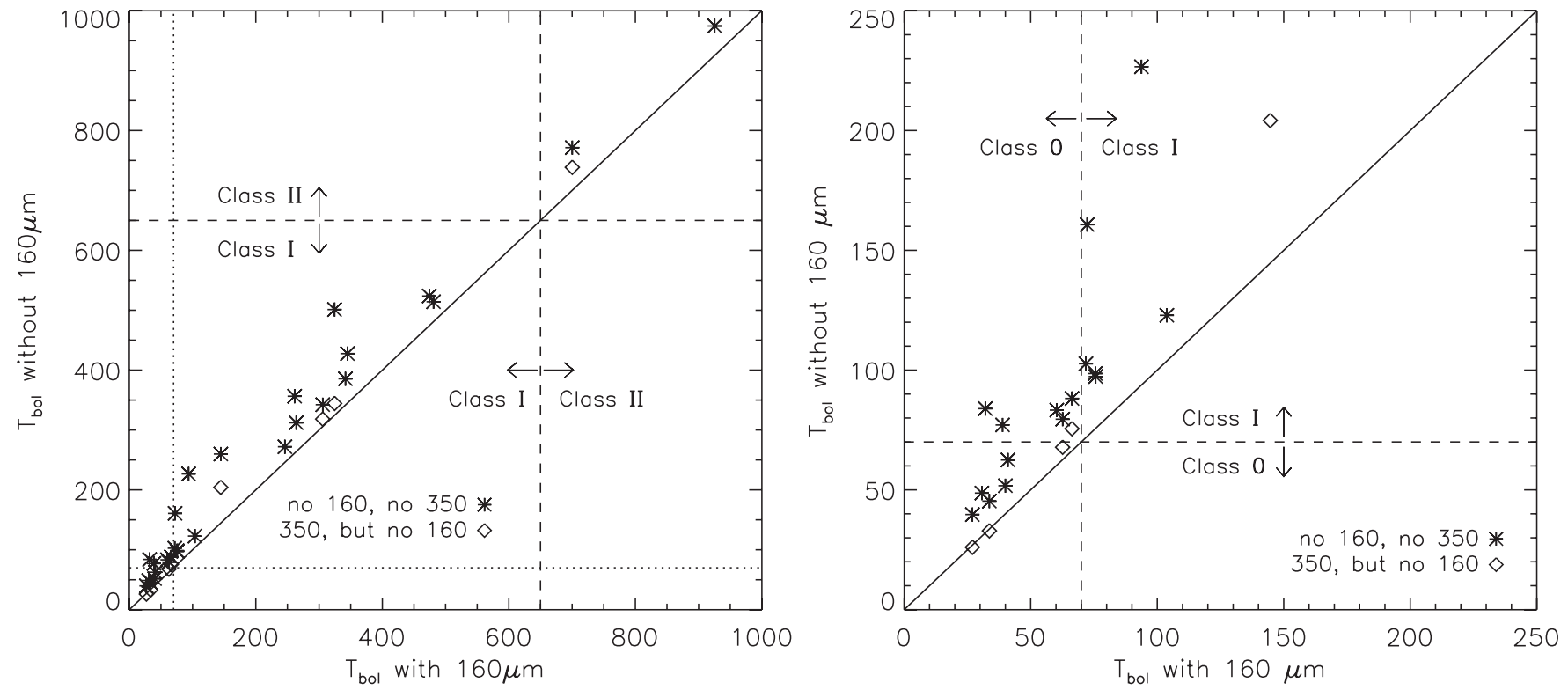

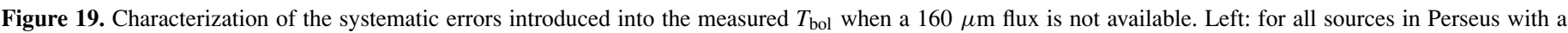

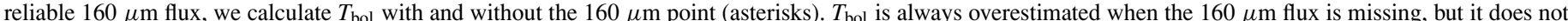

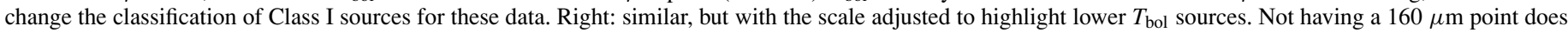

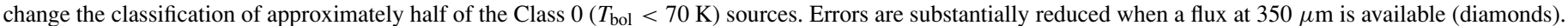

We first look at two example sources, one cold (IRAS 03282+3039; $\left.T_{\text {bol }} \sim 33 \mathrm{~K}\right)$ and one warmer $\left(T_{\text {bol }} \sim 163 \mathrm{~K}\right)$. IRAS $03282+3039$ is isolated, so we can use the IRAS fluxes without worrying about confusion due to the large IRAS beam. We calculate the "true" $L_{\text {bol }}$ and $T_{\text {bol }}$ by including the IRAS 60 and $100 \mu \mathrm{m}$ fluxes, a SHARC II $350 \mu \mathrm{m}$ flux, and a MIPS $160 \mu \mathrm{m}$ flux measured in a large aperture, which is almost two times higher than the PSF-fit flux. Using the underestimated point source $160 \mu \mathrm{m}$ flux, and no longer including IRAS or SHARC II data, causes an underestimate of $L_{\mathrm{bol}}$ by $35 \%$ and an overestimate of $T_{\text {bol }}$ by $15 \%$ compared to the "true" values. Omitting the $160 \mu \mathrm{m}$ point altogether, as would be appropriate for saturated sources, results in an underestimate of $L_{\mathrm{bol}}$ by $7 \%$ and an overestimate of $T_{\text {bol }}$ by $9 \%$.
Thus we conclude that while the $160 \mu \mathrm{m}$ point is important for characterization of the SED of embedded objects, our integration method can interpolate over a missing $160 \mu \mathrm{m}$ flux to estimate $L_{\text {bol }}$ and $T_{\text {bol }}$ to within $20 \%$ for cold sources. Severely underestimated $160 \mu \mathrm{m}$ flux densities will cause larger errors of up to $50 \%$. Using a similar procedure for the warmer source $\left(T_{\text {bol }}=163 \mathrm{~K}\right)$, we find that omitting the $160 \mu \mathrm{m}$ flux results in errors in the opposite sense compared to the colder source: an overestimate of $L_{\mathrm{bol}}$ by $28 \%$ and an underestimate of $T_{\mathrm{bol}}$ by $18 \%$.

For a more general result, we compare $T_{\text {bol }}$ calculated with the $160 \mu \mathrm{m}$ flux included in the SED to the value found by omitting the $160 \mu \mathrm{m}$ point, for all sources in Perseus with a measured $160 \mu \mathrm{m}$ flux (Figure 19, left panel). For this plot, we have 
adopted classifications from Chen et al. (1995), as discussed in Section 4. Although $T_{\text {bol }}$ calculated without the $160 \mu \mathrm{m}$ flux is always overestimated compared to $T_{\text {bol }}$ calculated with the $160 \mu \mathrm{m}$ flux, it is never a large enough effect to shift the source classification of objects with $T_{\text {bol }} \gtrsim 100 \mathrm{~K}$ (e.g., from Class I to Class II). This is not the case for Class 0 sources, however, as can be seen in the right panel of Figure 19, where the scale has been adjusted to highlight the lowest $T_{\text {bol }}$ values. Five sources that have $T_{\text {bol }}<70 \mathrm{~K}$ (Class 0 ) when including the $160 \mu \mathrm{m}$ flux are shifted to $650<T_{\text {bol }}>70 \mathrm{~K}$ (Class I) when the $160 \mu \mathrm{m}$ flux is omitted. If a published $350 \mu \mathrm{m}$ flux is available (diamonds), the errors in $T_{\text {bol }}$ resulting from excluding the $160 \mu \mathrm{m}$ flux are almost completely eliminated.

Based on Figures 17-19 and the above examples, we estimate overall uncertainties for measured $L_{\mathrm{bol}}$ and $T_{\mathrm{bol}}$ values of $20 \%-50 \%$, depending on whether or not a $160 \mu \mathrm{m}$ flux is available. If we are unable to measure a $160 \mu \mathrm{m}$ flux, $T_{\text {bol }}$ will almost certainly be an overestimate for very cold sources, which may affect our classification of the most deeply embedded protostars.

\section{REFERENCES}

Adams, F. C., Lada, C. J., \& Shu, F. H. 1987, ApJ, 312, 788

Alves, J., Lombardi, M., \& Lada, C. J. 2007, A\&A, 462L, 17

André, P. 1994, in The Cold Universe, ed. T. Montmerle, C. J. Lada, I. F. Mirabel, \& J. Tran Thanh Van (Gif-sur-Yvette: Editions Frontieres), 179

André, P., \& Montmerle, T. 1994, ApJ, 420, 837

André, P., Ward-Thompson, D., \& Barsony, M. 1993, ApJ, 406, 122

Aspin, C., Sandell, G., \& Russell, A. P. G. 1994, A\&AS, 106, 165

Beckwith, S. V. W., \& Sargent, A. I. 1996, Nature, 383, 139

Beckwith, S. V. W., Sargent, A. I., Chini, R. S., \& Güsten, R. 1990, AJ, 99, 924

Chabrier, G. 2003, PASP, 115, 763

Chen, H., Myers, P. C., Ladd, E. F., \& Wood, D. O. S. 1995, ApJ, 445, 377

Cieza, L., et al. 2007, ApJ, 667, 308

Cohen, M. 1980, AJ, 85, 29

Cohen, M., \& Kuhli, L. V. 1979, ApJS, 41, 743

Crapsi, A., van Dishoeck, E. F., Hogerheijde, M. R., Pontoppidan, K. M., \& Dullemond, C. P. 2008, A\&A, 486, 245

Di Francesco, J., Evans, N. J., II, Caselli, P., Myers, P. C., Shirley, Y., Aikawa, Y., \& Tafalla, M. 2007, in Protostars and Planets V, ed. B. Reipurth, D. Jewitt, \& K. Keil (Tucson, AZ: Univ. Arizona Press), 17

Dunham, M. M., Crapsi, A., Evans, N. J., II, Bourke, T. L., Huard, T. L., Myers, P. C., \& Kauffmann, J. 2008, ApJS, 179, 249

Ebert, R. 1955, Z. Astrophys., 37, 217

Enoch, M. L., Evans, N. J. II, Sargent, A. I., Glenn, J., Rosolowsky, E., \& Myers, P. C. 2008, ApJ, 684, 1240

Enoch, M. L., Glenn, J., Evans, N. J., II, Sargent, A. I., Young, K. E., \& Huard, T. L. 2007, ApJ, 666, 982

Enoch, M. L., et al. 2006, ApJ, 638, 293

Evans, N. J. II, et al. 2003, PASP, 115, 965

Evans, N. J., II, et al. 2007, Final Delivery of Data from the c2d Legacy Project: IRAC and MIPS (Pasadena, CA: SSC)

Evans, N. J., II, et al. 2009, ApJ, in press, arXiv: 0811.1059

Froebrich, D., Schmeja, S., Smith, M. D., \& Klessen, R. S. 2006, MNRAS, 368, 435

Greene, T. P., Wilking, B. A., André, P., Young, E. T., \& Lada, C. J. 1994, ApJ, 434,614

Greene, T. P., \& Young, E. T. 1992, ApJ, 395, 516
Habets, G. M. H. J., \& Heintze, J. R. W. 1981, A\&A, 46, 193

Hartmann, L., \& Kenyon, S. J. 1985, ApJ, 299, 462

Harvey, P. M., Merin, B., Huard, T. L., Rebull, L. M., Chapman, N., Evans,

N. J., II, \& Myers, P. C. 2007a, ApJ, 663, 1149

Harvey, P. M., et al. 2006, ApJ, 644, 307

Harvey, P. M., et al. 2007b, ApJ, 663, 1139

Hatchell, J., \& Fuller, G. 2008, A\&A, 482, 855

Hatchell, J., Fuller, G. A., \& Richer, J. S. 2007a, A\&A, 472, 187

Hatchell, J., Fuller, G. A., Richer, J. S., Harries, T. J., \& Ladd, E. F. 2007b, A\&A, 468, 1009

Herbig, G. H., \& Kameswara, R. N. 1972, ApJ, 174, 401

Indebetouw, R., Whitney, B. A., Johnson, K. E., \& Wood, K. 2006, ApJ, 636, 362

Jayawardhana, R., Hartmann, L., \& Calvet, N. 2001, ApJ, 548, 310

Jeans, J. H. 1928, Astronomy and Cosmogony (Cambridge: Cambridge Univ. Press), 340

Jørgensen, J. K., Bourke, T. L., Myers, P. C., Schöier, F. L., van Dishoeck, E. F., \& Wilner, D. J. 2005, ApJ, 631, L77

Jørgensen, J. K., Johnstone, D., Kirk, H., \& Myers, P. C. 2007, ApJ, 656, 293

Jørgensen, J. K., Johnstone, D., Kirk, H., Myers, P. C., Allen, L. E., \& Shirley, Y. L. 2008, ApJ, 683, 822

Jørgensen, J. K., et al. 2006, ApJ, 645, 1246

Kenyon, S. J., \& Hartmann, L. W. 1995, ApJS, 101, 117

Kenyon, S. J., Hartmann, L. W., Strom, K. M., \& Strom, S. E. 1990, AJ, 99, 869

Kirk, H., Johnstone, D., \& DiFrancesco, J. 2006, ApJ, 646, 1009

Lada, C. J. 1987, in IAU Symp. 115, Star Forming Regions, ed. M. Peimbert \& J. Jugaku (Dordrecht: Reidel), 1

Looney, L. W., Mundy, L. G., \& Welch, W. J. 2000, ApJ, 529, 477

Merín, B., et al. 2008, ApJS, 177, 551

McCaughrean, M. J., Rayner, J. T., \& Zinnecker, H. 1994, ApJ, 436, L189

Myers, P. C., Adams, F. C., Chen, H., \& Schaff, E. 1998, ApJ, 492, 703

Myers, P. C., \& Ladd, E. F. 1993, ApJ, 413, 47

Natta, A., Testi, L., Calvet, N., Henning, Th., Waters, R., \& Wilner, D. 2007, in Protostars and Planets V, ed. B. Reipurth, D. Jewitt, \& K. Keil (Tucson, AZ: Univ. Arizona Press), 767

Ossenkopf, V., \& Henning, Th. 1994, A\&A, 291, 943

Palla, F., \& Stahler, S. W. 1991, ApJ, 375, 288

Pringle, J. E. 1989, MNRAS, 239, 361

Rebull, L. M., et al. 2007, ApJS, 171, 447

Reipurth, B., \& Bally, J. 2001, ARA\&A, 39, 403

Robitaille, T. P., Whitney, B. A., Indebetouw, R., Wood, K., \& Denzmore, P. 2006, ApJS, 167, 256

Sandell, G., \& Knee, L. B. G. 2001, ApJ, 546, L49

Shirley, Y. L., Evans, N. J., II, \& Rawlings, J. M. C. 2002, ApJ, 575, 337

Shu, F. H. 1977, ApJ, 214, 488

Spezzi, L., et al. 2008, ApJ, 680, 1295

Tassis, K., \& Mouschovias, T. Ch. 2005, ApJ, 618, 783

Vieira, S. L. A., Corradi, W. J. B., Alencar, S. H. P., Mendes, L. T. S., Torres, C. A. O., Quast, G. R., Guimarães, M. M., \& da Silva, L. 2003, AJ, 126, 2971

Visser, A. E., Richer, J. S., \& Chandler, C. J. 2002, AJ, 124, 2756

Vorobyov, E. I., \& Basu, S. 2006, ApJ, 650, 956

Walawender, J., Bally, J., Kirk, H., \& Johnstone, D. 2005, AJ, 130, 1795

Walker, C. K., Lada, C. J., \& Young, E. T. 1986, ApJ, 309, L47

Ward-Thompson, D., André, P., Crutcher, R., Johnstone, D., Onishi, T., \& Wilson, C. 2007, in Protostars and Planets V, ed. B. Reipurth, D. Jewitt, \& K. Keil (Tucson, AZ: Univ. Arizona Press), 33

Whitney, B. A., Wood, K., Bjorkman, J. E., \& Cohen, M. 2003, ApJ, 598, 1079

Wu, J., Dunham, M. M., Evans, N. J. II, Bourke, T. L., \& Young, C. H. 2007, AJ, 133,1560

Young, C. H., \& Evans, N. J., II. 2005, ApJ, 627, 293

Young, C. H., Shirley, Y. L., Evans, N. J., II, \& Rawlings, J. M. C. 2003, ApJS, 145,111

Young, K. E., et al. 2006, ApJ, 644, 326 